Testes de hipóteses para componentes de variância utilizando estatísticas $\mathrm{U}$

Juvêncio Santos Nobre

TESE APRESENTADA

$\mathrm{AO}$

INSTITUTO DE MATEMÁTICA E ESTATÍSTICA

DA

UNIVERSIDADE DE SÃO PAULO

PARA

OBTENÇÃO DO GRAU DE DOUTOR

EM

CIÊNCIAS

Área de Concentração: Estatística

Orientador: Julio da Motta Singer

Durante a elaboração deste trabalho o autor recebeu apoio financeiro do CNPq

- São Paulo, Agosto de 2007 - 


\section{Testes de hipóteses para componentes de variância utilizando estatísticas $\mathrm{U}$}

Este exemplar corresponde à redação final da tese devidamente corrigida e defendida por Juvêncio Santos Nobre e aprovada pela comissão julgadora.

São Paulo, 09 de Agosto de 2007.

Comissão Julgadora:

- Prof. Dr. Julio da Motta Singer (Orientador)- IME/USP

- Prof. Dr. Nelson Ithiro Tanaka- IME/USP

- Prof. Dr. Aluísio de Souza Pinheiro - IMECC/UNICAMP

- Prof. Dr. Francisco José de Azevedo Cysneiros - DE/UFPE

- Prof. Dr. Mário de Castro Andrade -ICMC/USP 
"I turn away in fright and horror from this lamentable plague, of sequences random variables which do not satisfy the CLT".

Juvêncio S. Nobre

"Wir müssen wissen,

Wir werden wissen".

David Hilbert 


\section{"Sou matuto do Nordeste criado dentro da mata cabloco cabra da peste poeta cabeça chata". Patativa do Assaré}

"Eu sou de uma terra que o povo padece

Mas não esmorece e procura vencer. Da terra querida, que a linda cabocla De riso na boca zomba no sofrê Não nego meu sangue, não nego meu nome. Olho para a fome, pergunto: que há? Eu sou brasileiro, filho do Nordeste, Sou cabra da Peste, sou do Ceará". Patativa do Assaré

"Cabeça chata sim quadrada não!". Autor desconhecido 
Dedico este trabalho

A Deus acima de tudo

À minha mãe Gracilene, por ser o melhor pãe (pai e mãe) do mundo À minha esposa Jacqueline, por existir na minha vida Aos meus mestres e amigos Prof. Maurício, Prof. Julio e Prof. Dalton, obrigado por tudo Para os que lutam por um Ceará melhor À minha vozinha Ana Batista (In memoriam) 


\section{Agradecimentos}

Gostaria de agradecer:

A Deus, por me oferecer saúde, disposição, discernimento e por colocar várias pessoas maravilhosas na minha vida, além de me fornecer inúmeras oportunidades. A Ele só tenho a agradecer por tudo.

Agradeço à pessoa a quem tenho plena consciência de dever tudo que sou hoje: Gracilene, meu super pãe (pai e mãe): grande responsável por muito do que acontece de bom na minha vida, um exemplo de bom caráter, determinação e perseverança. Obrigado, por todo amor destinado, palavras de conforto, ensinamentos, a força dada e por sempre incentivar, confiar e acreditar em mim, mesmo nos momentos em que nem eu acreditava. Obrigado Mãezinha, este momento é a realização de mais um sonho, que por inúmeras vezes pareceu impossível, por este motivo, dedico este trabalho à senhora.

Ao meu orientador, mestre e amigo, professor Julio da Motta Singer, um profissional excepcional, um orientador sui generis, uma pessoa com um caráter exemplar. Agradeço pela grande oportunidade de ter sido seu orientando, pelo grande entusiasmo, por sempre acreditar no meu potencial (muitas vezes latente) mesmo nos inúmeros momentos improdutivos, pela paciência e por todos os ensinamentos (que não ficam restritos somente à vida acadêmica) transmitidos nos 5 anos em que estive na cidade de São Paulo.

Ao meu grande mestre, professor João Maurício Araújo Mota, muito mais que um professor: Um grande mestre, amigo e pai. Muito obrigado por sempre acreditar e investir em mim, pela ajuda imensurável desde o começo da graduação até os dias de hoje. Tenho o senhor como um grande exemplo de profissional e ser humano.

I am grateful to professor Pranab K. Sen for his constructive comments and suggestions which helped in the development of this thesis.

Ao professor Dalton, um grande exemplo de professor, mestre e amigo. Obrigado pelo incentivo constante, apoio incomensurável, principalmente nas grandes decisões da minha vida acadêmica. 
Ao amigo e professor João Welliandre pela alegria, amizade, confiança e incentivo que sempre encontro no senhor.

À minha amada esposa Jacqueline (meu Teorema Limite Central Universal), obrigado pela grande paciência (vai para o céu direto sem pegar fila!!!!), pelos inúmeros sonhos compartilhados (alguns já realizados), pelo companherismo constante, pelo amor e força a mim destinados em todos os momentos. Todo dia agradeço ao nosso bom Deus por ter colocado você para colorir a minha vida.

Aos meus sogros Maria do Socorro e Otacílio Bandeira e ao meu cunhado Júlio por toda consideração. À minha vozinha Ana Batista (in memoriam) por me adotar como um verdadeiro neto e por sempre dedicar muita atenção e carinho nos momentos em que estivemos juntos.

Aos professores Aluísio Pinheiro e Hildete Pinheiro (IMECC-Unicamp), pelos esclarecimentos que me ajudaram de sobremaneira para a conclusão da proposta desta tese. Muito obrigado!!

Aos membros da banca examinadora pelas sugestões e comentários valiosos para o aprimoramento do texto.

Uma menção mais do que especial aos amigos Frederico Poleto, Alexandre Patriota e Francisco Marcelo, os dois primeiros por me ajudarem a implementar computacionalmente o teste desenvolvido e o terceiro por revisar e discutir trechos do presente texto. Agradeço também a Luciana Godoi pela discussão sobre a distribuição t assimétrica.

Aos meus padrinhos Fátima e Tácito por todo o suporte dado a minha mãe e a mim, principalmente durante a infância. À Maria Medeiros, Terezinha de Campos Modesto (in memoriam), tia Jack, tio Afonso e tia Goreti pela ajuda, palavras de incetivo e motivação nos momentos em que eu mais precisava.

A todos professores e funcionários do Departamento de Estatística e Matemática Aplicada da Universidade Federal do Ceará, Dentre os professores, gostaria de agradecer: João Maurício, Rosa Salani, João Welliandre, Nelson Braga (in memoriam), Ronald Nojosa, Júlio Barros, Sílvia Freitas, Ana Maria, Robson Medeiros, André Jalles e Manoel Campelo; as funcionárias: Margarida, Margéri, Luisa e Mariluse; aos grandes amigos que formei durante meus quatro anos de graduação: Caio, Carlos, Dhavynci, Agnaldo, Cledinaldo e Adriana; aos amigos e colegas que ingressaram na turma de $\mathbf{1 9 9 8 . 1}$ e todos os demais amigos e colegas que certamente ficaram torcendo por mim nesse grande desafio em São Paulo. Obrigado pela amizade e força que vocês me deram e tenho certeza que todos torceram pelo êxito desta jornada.

A todos os professores do Departamento de Estatística do IME-USP, que contribuíram 
com o aperfeiçoamento de minha formação, em especial: Antônio Carlos, Antônio Galves, Caio Dantas, Carlos Alberto Bragança, Chang Chiann, Denise Botter, Elisabeth Kira, Fábio Prates, Heleno Bolfarine, Júlia Soler, Julio Singer, Gilberto Alvarenga, Márcia Branco, Nelson Tanaka, Serguei Popov, Sílvia Ferrari, Sílvia Nagib, Viviana Giampaoli e Wagner Borges e as funcionárias: Simone, Cecília, Helena, Lourdes, Vanessa (ABE), Sílvia (CEA) e Elaine que sempre estão dispostas a ajudar com muita simpatia e competência. Agradeço também a todos os funcionários da CPG e da biblioteca do IME/USP, da xerox e do áudiovisual por sempre atenderem com prontidão.

À comunidade cearense (alguns naturalizados) no IME/USP, também denominada como Rapadura Estocástica: Alexandre, Caio, Gilberto, Ítalo, Jacqueline, Jony, Rafael, Ronald, Marcelo, Michel e Patrícia. Agradeço pela amizade e pela oportunidade de treinar constantemente o meu cearês.

A todos meus amigos (desculpas antecipadas caso eu esqueça de alguém), em especial para meu grande irmão Caio (pela sincera amizade, apoio constante e pela enorme ajuda no começo desta jornada) e Dona Verônica, Michelli e Horácio (por toda amizade e consideração), (compadre) Marcelo e Lane (por serem verdadeiros irmãos), Rafael (Jovem) e Michel (pela amizade, brincadeiras e por sempre deixarem eu ganhar no WE), Rogério e família (por sempre fazer eu me sentir em casa, mesmo estando tão longe...), Sr. Zé Airton e Sra. Gizelane (por me receberem tão bem no pedacinho do Ceará que criaram aqui em SP), Perseverando e Edijane, Diana (Colômbia), Elier, Raydonal e Patrícia, Ronald e Fran, Gilberto e Regelli, Alexandre, Thiago Rodrigo (pela amizade e pelas idas aos jogos do Vozão) e Dona Lúcia, Fred e Lilian, Tatiane e Alessandro, Diana e Gustavo, Marcelo (RJ), Cléber, Iracema, Kelly, Paulão, Luz Mery, Felipe Osório, Jorge Bázan, Ivan, Carinne, Jony, Patrícia (eu digo é valha), Núbia, Luz Marina, Francisco e Audrey, Michel (PE), Waldemar (PE), Gérman, Estevão e Arthur. Um agradecimento especial aos amigos André Barros (CE), pela grande ajuda no período que antecedeu as provas da segunda fase do vestibular da UFC, e a Prof ${ }^{a}$ de Química do Liceu do Ceará Júlia Maria por abrir meus olhos para a realidade.

Aos meus colegas da época do CRUSP: Caio, Rafael, Alexandre, Álvaro, Marcelo obrigado pela excelente convivência e pelo clima de camaradagem. Agradeço também a minha assistente social Neusa.

E a todos que não mencionei que me ajudaram (direta ou indiretamente) na realização de mais um sonho.

Nobre, Juvêncio S. 


\section{Resumo}

Nós consideramos decomposições de estatísticas $U$ para obter testes para componentes de variância. As distribuições assintóticas das estatísticas de testes sob a hipótese nula são obtidas supondo apenas a existência do quarto momento do erro condicional e do segundo momento dos efeitos aleatórios. Isso permite sua utilização em uma classe bastante ampla de distribuições. Sob a suposição adicional de existência do quarto momento dos efeitos aleatórios, obtemos também a distribuição assintótica das estatísticas sob uma seqüência de hipóteses alternativas locais. Comparamos a eficiência dos testes propostos com aqueles dos testes clássicos, obtidos sob suposição de normalidade, por meio de estudos de simulação. Os testes propostos se mostram mais adequados nas situações em que a amostra é de tamanho moderado ou grande, independentemente da distribuição das fontes de variação, e nas situações em que existe fortes afastamentos da normalidade.

Palavras-chave: componentes de variância, hipóteses não regulares, modelos lineares mistos, estatística $U$, martingais. 


\begin{abstract}
We consider decompositions of U-statistics to obtain tests for null variance components in linear mixed models. Their asymptotic distributions under the null hypothesis are obtained only assuming the existence of the first four moments of the conditional error distribution and the existence of the first two moments of the random effects distribution. Thus, the proposed U-tests may be employed in a large class of models. Under the additional assumption of the existence of the fourth moment of the distribution of the random effects, we also obtain the asymptotic distribution of the U-tests under a sequence of local hypothesis. We compare their efficiency with that of classical tests derived under the assumption of normality, through simulation studies. The proposed tests are more efficient in situations where the sample size is moderate or large, independently of the distribution of the sources of variation; they also perform better in situations where the underlying distributions are far from normal.
\end{abstract}

Keywords: variance components, nonstandard hypothesis, linear mixed models, $U$-statistics, martingales. 


\section{Lista de Tabelas}

1.1 Índice de placa bacteriana. . . . . . . . . . . . . . . . . . . . 2

1.2 Diâmetro sistólico da aorta por unidade de peso $(\mathrm{mm} / \mathrm{kg})$ para o grupo de 32 recém-nascidos pré-termo PIG. . . . . . . . . . . . . . . . 4

3.1 Estimativas do poder do teste $(\%)$ de $\mathcal{H}_{0}: \sigma_{b}^{2}=0$ sob o modelo (3.1). . . . 25

3.2 Estimativas do poder do teste $(\%)$ de $\mathcal{H}_{0}: \sigma_{b}^{2}=0$ sob o modelo (3.1) para um estudo balanceado, com 5 observações por unidade amostral. . . . . . . . .

3.3 Estimativas do poder do teste $(\%)$ de $\mathcal{H}_{0}: \sigma_{b}^{2}=0$ sob o modelo (3.1) para um estudo desbalanceado, com o número de observações por unidade amostral sendo selecionado de forma equiprovável no conjunto $\{5,6,7,8,9,10\}$. . . .

3.4 Estimativas do poder do teste (\%) de $\mathcal{H}_{0}: \sigma_{b}^{2}=0$ sob o modelo (3.1) para um estudo desbalanceado, com o número de observações por unidade amostral sendo selecionado segundo uma distribuição $2+\mathrm{G}(0,15)$. . . . . . . . . . .

3.5 Estimativas do poder do teste $(\%)$ de $\mathcal{H}_{0}: \sigma_{b}^{2}=0$ sob o modelo (3.1) para um estudo balanceado, com 5 obervações por unidade amostral, considerando distribuições assimétricas com caudas pesadas para o efeito aleatório e erro

3.6 Estimativas do poder do teste $(\%)$ de $\mathcal{H}_{0}: \sigma_{b}^{2}=0$ sob o modelo (3.29) em estudos balanceados, sob normalidade do erro condicional. . . . . . . . . . .

3.7 Estimativas do poder do teste $(\%)$ de $\mathcal{H}_{0}: \sigma_{b}^{2}=0$ sob o modelo (3.29) em estudos balanceados, com $e_{i j} \sim t_{5} \times \sqrt{3 / 5} \ldots \ldots \ldots$

3.8 Estimativas do poder do teste $(\%)$ de $\mathcal{H}_{0}: \sigma_{b}^{2}=0$ no modelo (3.29) para um estudo balanceado, com 5 observações por unidade amostral, sob normalidade. 37

3.9 Estimativas do poder do teste $(\%)$ de $\mathcal{H}_{0}: \sigma_{b}^{2}=0$ no modelo (3.29) para um estudo desbalanceado, com o número de observações por unidade amostral sendo selecionado de forma equiprovável no conjunto $\{5,6,7,8,9,10\}$, com distribuições com caudas pesadas para o efeito aleatório e o erro condicional. 
3.10 Estimativas do poder do teste $(\%)$ de $\mathcal{H}_{0}: \sigma_{b}^{2}=0$ no modelo (3.29) para um estudo desbalanceado, com o número de observações por unidade amostral sendo selecionado segundo uma distribuição $2+\mathrm{G}(0.15)$, sob normalidade. 38

3.11 Estimativas do poder do teste $(\%)$ de $\mathcal{H}_{0}: \sigma_{b}^{2}=0$ no modelo (3.1), para um estudo balanceado, com 5 obervações por unidade amostral, considerando distribuições assimétricas com caudas pesadas para o efeito aleatório e erro

3.12 Estimativas do poder do teste $(\%)$ de $\mathcal{H}_{0}: \sigma_{1}^{2}=0$ e $\sigma_{0}^{2}>0$ no modelo (3.50) para um estudo balanceado, com 5 observações por unidade amostral, sob normalidade. . . . . . . . . . . . . . . . . .

3.13 Estimativas do poder do teste $(\%)$ de $\mathcal{H}_{0}: \sigma_{1}^{2}=0$ e $\sigma_{0}^{2}>0$ no modelo (3.50) para um estudo balanceado, com 5 observações por unidade amostral, considerando distribuições assimétricas com caudas pesadas independentes para os efeitos aleatórios. . . . . . . . . . . . . . . . . . . . . 46

4.1 Estimativas ( \pm erros-padrão estimados) dos parâmetros e impacto percentual do modelo (4.3) removendo as crianças \#12 and \#29. . . . . . . . . . . . . . 49

4.2 Testes para componentes de variância no modelo (4.3) removendo as crianças \#12 e \#29. . . . . . . . . . . . . . . . . . . . . . . . . . . . . . . . . . . . . . . . . . 49

4.3 Testes para componentes de variância no modelo (4.4) . . . . . . . . . . . . 52

4.4 Estimativas dos parâmetros do modelo (4.4) . . . . . . . . . . . . . . . . . 52 


\section{Lista de Figuras}

1.1 Diagramas de dispersão entre os índices de placa bacteriana pré-escovação e pós-escovação. . . . . . . . . . . . . . . . . . . . 3

1.2 Gráfico de perfis individuais e médio para o diâmetro sistólico da aorta por unidade de peso $(\mathrm{mm} / \mathrm{kg})$ para o grupo de 32 recém-nascidos pré-termo PIG.

4.1 Histograma para EBLUP padronizado. . . . . . . . . . . . . . . . 51

4.2 Envelope simulado com 95\% e histograma para o resíduo com confundimento mínimo padronizado. . . . . . . . . . . . . . . . . . . 51 
Agradecimentos vi vi vis

Resumo ix

$\begin{array}{ll}\text { Abstract } & \mathbf{x}\end{array}$

Lista de Tabelas $\quad$ xi

Lista de Figuras $\quad$ xiii

1 Introdução $\quad 1$

1.1 Motivação . . . . . . . . . . . . . . . . . . . . . . 1

1.2 Especificação do modelo . . . . . . . . . . . . . . . . . . . 4

1.3 Testes para componentes de variância . . . . . . . . . . . . . 6

2 Estatísticas U $\quad 11$

3 Testes para componentes de variância $\quad 17$

3.1 Introdução . . . . . . . . . . . . . . . . . . . . . . . . . . . . . . 17

3.2 Modelo de ANOVA com um único efeito aleatório . . . . . . . . . . . . . . . 17

3.2 .1 Estudos de Simulação . . . . . . . . . . . . . . . . . . . . . . . 24

3.2 .2 Comparação entre os testes $F$ e $J_{n} \ldots \ldots \ldots$. . . . . . . 26

3.3 Modelos com intercepto aleatório . . . . . . . . . . . . . . . . . . 31

3.3 .1 Estudos de Simulação . . . . . . . . . . . . . . . . . . . 33

3.3.2 Comparação entre os testes $F_{D}$ e $J_{n \widehat{\beta}} \ldots \ldots$. . . . . . . . . . 34

3.4 Modelos com intercepto e inclinação aleatórios . . . . . . . . . . . . . . . . 39

3.4.1 Estudo de simulação . . . . . . . . . . . . . . . . . . . . . . . . . 44

4 Aplicações $\quad 47$ 
4.1 Análise do Exemplo $1.1 \ldots \ldots \ldots$. . . . . . . . . . . . . . . . 47

4.2 Análise do Exemplo $1.2 \ldots \ldots$. . . . . . . . . . . . . . . 50

5 Considerações finais $\quad 53$

5.1 Conclusões . . . . . . . . . . . . . . . . . . . . . . 53

5.2 Pesquisas futuras . . . . . . . . . . . . . . . . . . 54

A Resultados básicos $\quad 55$

B Demonstrações $\quad 56$

B.1 Expressão (3.15) . . . . . . . . . . . . . . . . . . . 56

B.2 Identidades $(3.17)$ e $(3.18) \ldots \ldots \ldots \ldots \ldots \ldots$

B.3 Teorema $3.1 \ldots \ldots \ldots \ldots \ldots \ldots \ldots$

B.4 Teorema $3.2 \ldots \ldots \ldots \ldots \ldots$

B.5 MINQUE de $\sigma_{e}^{2} \ldots \ldots \ldots \ldots \ldots \ldots$. . . . . . . . . . . . . . . . . . . . . . . . .

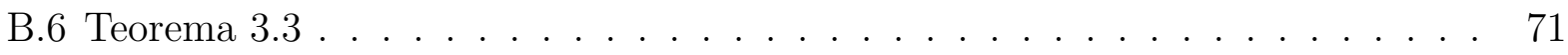

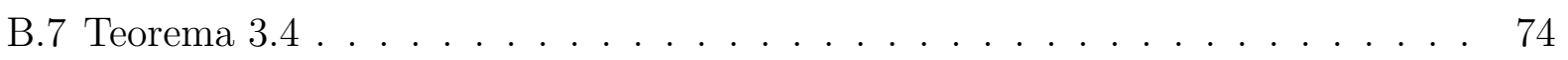

B.8 Demonstração das condições de regularidade $(3.45) \ldots \ldots$. . . . . . . . . . 77

B.9 Cálculo de $\mathbb{E}\left[J_{n \beta}^{*}\right] \ldots \ldots \ldots$. . . . . . . . . . . . . . . . . . . . . . 79

B.10 Teorema $3.5 \ldots \ldots \ldots \ldots \ldots \ldots \ldots$

B.11 Teorema $3.6 \ldots \ldots \ldots \ldots \ldots$. . . . . . . . . . . . . . . . . . . . .

$\begin{array}{ll}\text { Referências Bibliográficas } & 84\end{array}$ 


\section{Capítulo 1}

\section{Introdução}

\subsection{Motivação}

Em estudos com medidas repetidas, cada unidade amostral é observada em pelo menos duas ocasiões, e por essa razão, esperamos uma dependência das medidas intra-unidades amostrais. Estudos desse tipo são comuns em pesquisas de diversas áreas, como Agricultura, Biologia, Educação, Medicina, Economia, etc. Eles abrangem, entre outros, delineamentos com parcelas subdivididas ("split-plot"), delineamentos com intercâmbio ("crossover") e estudos longitudinais. Para detalhes, veja Lindsey (1993), Singer e Andrade (2000), Verbeke e Molenberghs (2000), Diggle et al. (2002) e Molenberghs e Verbeke (2005). A seguir, apresentamos alguns exemplos de estudos com medidas repetidas.

Exemplo 1.1 (Índice de placa bacteriana): Um estudo longitudinal foi realizado na Faculdade de Odontologia da Universidade de São Paulo, para comparar dois tipos de escova de dentes (monobloco e convencional) com relação a eficácia na remoção de placa bacteriana, utilizando ou não dentifrício, e também quanto à manutenção da capacidade de remoção de placa bacteriana (durabilidade) sob uso diário. Com esta finalidade, foram observadas 32 crianças em 4 sessões quinzenais, uma das quais correspondente à avaliação inicial. As crianças foram alocadas a dois grupos de tamanhos iguais, cada um submetido ao tratamento com uma das escovas. Durante o período de observação, cada criança utilizou a mesma escova que lhe foi dada na primeira sessão. Em cada sessão de avaliação, mediu-se um índice de placa bacteriana antes (pré-tratamento) e depois (pós-tratamento) da escovação. Os dados encontram-se na Tabela 1.1; uma análise desses dados está apresentada em Nobre (2004). A Figura 1.1 contêm gráficos de dispersão entre os índices de placa bacteriana pré-tratamento $(x)$ e pós-tratamento $(y)$. As linhas tracejadas vertical e horizontal correspondem, respectivamente, à média dos índices de placa pré e pós escovação para cada tipo de escova nas sessões de avaliação. O que caracteriza os dados desse estudo como longitudinais é a observação das mesmas unidades experimentais (crianças) ao longo das quatro sessões de avaliação. 
Tabela 1.1 Índice de placa bacteriana.

\begin{tabular}{|c|c|c|c|c|c|c|c|c|c|}
\hline \multirow[b]{2}{*}{ Criança } & \multirow[b]{2}{*}{ Escova } & \multicolumn{2}{|c|}{$1^{\underline{a}}$ sessão } & \multicolumn{2}{|c|}{$2^{\underline{a}}$ sessão } & \multicolumn{2}{|c|}{$3^{\underline{a}}$ sessão } & \multicolumn{2}{|c|}{$4^{\underline{a}}$ sessão } \\
\hline & & Antes & Depois & Antes & Depois & Antes & Depois & Antes & Depois \\
\hline 1 & Convencional & 1,05 & 1,00 & 1,13 & 0,84 & 1,15 & 0,86 & 1,13 & 0,94 \\
\hline 2 & Convencional & 1,07 & 0,62 & 0,92 & 0,62 & 1,02 & 0,57 & 1,15 & 0,85 \\
\hline 3 & Convencional & 0,82 & 0,62 & 1,52 & 1,07 & 1,39 & 0,97 & 1,78 & 1,39 \\
\hline 4 & Convencional & 1,37 & 0,90 & 1,65 & 1,20 & 1,75 & 1,40 & 1,92 & 1,67 \\
\hline 5 & Convencional & 1,97 & 1,52 & 1,30 & 1,07 & 1,50 & 1,15 & 1,65 & 1,37 \\
\hline 6 & Convencional & 1,30 & 0,82 & 1,17 & 0,70 & 0,75 & 0,50 & 1,47 & 1,12 \\
\hline 7 & Convencional & 1,61 & 1,19 & 1,52 & 1,13 & 1,22 & 1,00 & 1,63 & 1,22 \\
\hline 8 & Convencional & 1,02 & 0,73 & 1,08 & 0,64 & 0,94 & 0,73 & 1,14 & 0,97 \\
\hline 9 & Convencional & 1,62 & 1,25 & 1,45 & 1,10 & 1,10 & 0,75 & 1,70 & 1,32 \\
\hline 10 & Convencional & 1,65 & 1,22 & 1,57 & 1,22 & 1,47 & 1,10 & 1,62 & 1,17 \\
\hline 11 & Convencional & 1,02 & 0,78 & 0,60 & 0,47 & 0,88 & 0,75 & 1,36 & 1,08 \\
\hline 12 & Convencional & 0,71 & 0,60 & 1,13 & 0,39 & 0,84 & 0,65 & 1,65 & 1,31 \\
\hline 13 & Convencional & 1,70 & 1,55 & 1,85 & 1,37 & 1,87 & 1,55 & 1,60 & 1,30 \\
\hline 14 & Convencional & 1,30 & 1,02 & 1,65 & 0,97 & 1,72 & 1,20 & 1,37 & 1,22 \\
\hline 15 & Convencional & 1,40 & 0,80 & 1,83 & 1,03 & 1,76 & 1,38 & 1,96 & 1,15 \\
\hline 16 & Convencional & 1,40 & 1,12 & 1,25 & 0,67 & 1,50 & 1,10 & 1,50 & 1,22 \\
\hline 17 & Monobloco & 1,66 & 1,63 & 1,36 & 1,16 & 1,52 & 0,88 & 1,41 & 1,20 \\
\hline 18 & Monobloco & 1,02 & 0,80 & 0,92 & 0,82 & 1,10 & 0,76 & 1,28 & 1,15 \\
\hline 19 & Monobloco & 0,75 & 0,67 & 1,00 & 0,92 & 1,00 & 0,87 & 1,15 & 1,10 \\
\hline 20 & Monobloco & 1,29 & 1,23 & 0,91 & 0,76 & 1,14 & 0,94 & 1,35 & 0,97 \\
\hline 21 & Monobloco & 1,27 & 1,20 & 1,20 & 0,95 & 1,10 & 1,00 & 1,37 & 1,17 \\
\hline 22 & Monobloco & 1,07 & 0,85 & 1,39 & 1,25 & 1,39 & 1,25 & 1,28 & 1,21 \\
\hline 23 & Monobloco & 1,35 & 1,21 & 1,42 & 1,17 & 1,42 & 1,19 & 1,42 & 1,23 \\
\hline 24 & Monobloco & 1,32 & 1,02 & 1,60 & 1,40 & 1,35 & 1,02 & 1,50 & 1,25 \\
\hline 25 & Monobloco & 1,66 & 1,61 & 1,50 & 1,36 & 1,72 & 1,41 & 1,69 & 1,44 \\
\hline 26 & Monobloco & 1,30 & 1,07 & 0,84 & 0,61 & 0,88 & 0,61 & 0,96 & 0,57 \\
\hline 27 & Monobloco & 1,57 & 1,20 & 1,50 & 1,07 & 1,15 & 1,00 & 1,25 & 1,05 \\
\hline 28 & Monobloco & 1,67 & 1,50 & 1,47 & 1,32 & 1,07 & 0,97 & 1,50 & 1,37 \\
\hline 29 & Monobloco & 0,91 & 0,67 & 0,96 & 0,62 & 1,09 & 0,53 & 1,12 & 0,37 \\
\hline 30 & Monobloco & 1,06 & 0,70 & 1,00 & 0,85 & 1,15 & 0,93 & 1,12 & 1,00 \\
\hline 31 & Monobloco & 2,30 & 2,00 & 1,37 & 1,25 & 1,40 & 1,32 & 2,15 & 1,90 \\
\hline 32 & Monobloco & 1,15 & 1,00 & 1,23 & 1,11 & 1,15 & 1,07 & 1,26 & 1,00 \\
\hline
\end{tabular}

Nobre, Juvêncio S. 
Figura 1.1 Diagramas de dispersão entre os indices de placa bacteriana pré-escovação e pósescovação.

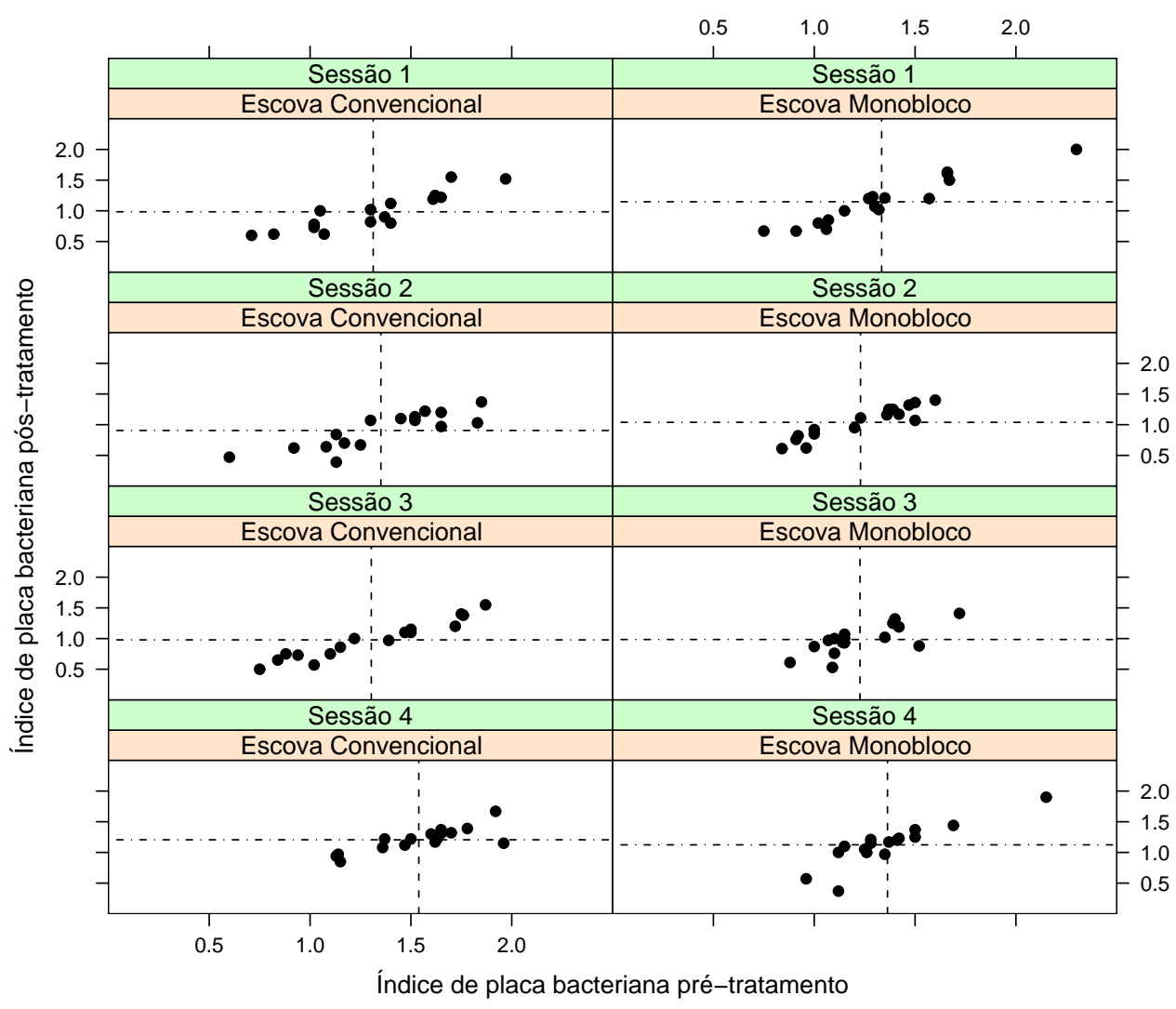

Exemplo 1.2 (Diâmetro da aorta): Um estudo longitudinal realizado na Faculdade de Medicina da Universidade de São Paulo, com o objetivo de avaliar a evolução de um conjunto de características cardio-circulatórias em recém-nascidos pré-termo (RNPT) e comparar os valores correspondentes a 39 semanas da concepção com aqueles obtidos de recém-nascidos a termo (com 39 semanas de gestação). Detalhes adicionais sobre o estudo podem ser obtidos em Afiune (2000) ou em Afiune et al. (2005). Aqui, concentraremos nossa atenção na análise do diâmetro sistólico da aorta por unidade de peso $(\mathrm{mm} / \mathrm{kg})$ para o grupo de 32 recémnascidos pré-termo e classificados como pequenos para a idade gestacional (PIG), cujos dados estão esquematicamente representados na Tabela 1.2. Na Figura 1.2, apresentamos o gráfico de perfis individuais e médio correspondentes.

Grande parte do esforço empregado na análise de dados com medidas repetidas está relacionada com a modelagem da estrutura de dependência intra-unidades amostrais. Uma alternativa bastante difundida para esse fim é a inclusão de variáveis latentes (não observáveis), comumente denominadas efeitos aleatórios, nos modelos utilizados na análise. 
Tabela 1.2 Diâmetro sistólico da aorta por unidade de peso $(\mathrm{mm} / \mathrm{kg})$ para o grupo de 32 recémnascidos pré-termo PIG.

\begin{tabular}{|c|c|c|c|c|c|c|c|c|c|c|c|c|c|c|}
\hline \multirow[b]{2}{*}{ RNPT } & \multicolumn{14}{|c|}{ Semanas pós-concepção } \\
\hline & 27 & 28 & 29 & 30 & 31 & 32 & 33 & 34 & 35 & 36 & 37 & 38 & 39 & 40 \\
\hline 1 & & & & & 7,2 & 6,8 & & 5,5 & & & 4,7 & & & \\
\hline 2 & & & & & & 7,1 & 8,0 & 7,7 & & & 6,5 & 5,6 & & \\
\hline 3 & & & & 7,4 & 8,3 & 9,4 & 10,0 & 9,2 & & & 8,0 & & & \\
\hline 4 & & & & & 7,7 & & 6,6 & & 5,5 & & 4,6 & & & \\
\hline 5 & & & & & & & 6,5 & & & & & 4,4 & & \\
\hline 6 & & & 7,6 & 8,6 & 9,3 & 8,0 & & 6,6 & & 5,0 & & 4,7 & & \\
\hline 7 & & & 6,6 & 8,4 & & 8,2 & 7,6 & & 6,6 & & & & & \\
\hline 8 & & & & & 7,1 & 6,3 & & & 6,1 & 5,9 & 5,7 & & 4,8 & \\
\hline 9 & & & & 8,5 & 8,4 & & & & & 4,9 & & & & \\
\hline 10 & & 8,3 & 7,4 & & 6,2 & & & 4,6 & & 3,8 & & & & \\
\hline 11 & & 9,8 & & & 9,1 & & 7,3 & & 5,3 & & & & & \\
\hline . & 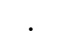 & . & 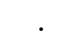 & & . & . & 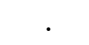 & . & & . & . & $\theta^{\circ}$ & . & \\
\hline . & & . & . & & . &. & . & . & & . & . & . & . & . \\
\hline 31 & & 8,5 & & & & & & & & & & & & \\
\hline 32 & & & 10,9 & 10,7 & 9,4 & & 8,0 & & & 5,8 & & 4,9 & & \\
\hline
\end{tabular}

Esses modelos são denominados modelos mistos ou modelos de efeitos aleatórios, e podem ser lineares ou não-lineares. Neste trabalho, considerar-se-á apenas a classe dos modelos lineares mistos.

\subsection{Especificação do modelo}

Modelos lineares mistos podem ser escritos na forma

$$
\mathbf{Y}_{i}=\mathbf{X}_{i} \boldsymbol{\beta}+\mathbf{Z}_{i} \mathbf{b}_{i}+\mathbf{e}_{i}, \quad i=1, \ldots, k
$$

em que $\mathbf{Y}_{i}$ representa o vetor $\left(n_{i} \times 1\right)$ de respostas da $i$-ésima unidade amostral, $\boldsymbol{\beta}$ é o vetor $(p \times 1)$ de parâmetros (efeitos fixos), $\mathbf{X}_{i}$ é a matriz $\left(n_{i} \times p\right)$ de especificação (conhecida e de posto completo) dos efeitos fixos, $\mathbf{b}_{i}$ é o vetor $(q \times 1)$ de variáveis latentes, que refletem o comportamento individual da $i$-ésima unidade amostral, $\mathbf{Z}_{i}$ é a matriz $\left(n_{i} \times q\right)$ de especificação (conhecida e de posto completo) dos efeitos aleatórios e $\mathbf{e}_{i}$ é um vetor $\left(n_{i} \times 1\right)$ de erros de medida aleatórios. Fazendo $\mathbf{Y}=\left(\mathbf{Y}_{1}^{\top}, \ldots, \mathbf{Y}_{k}^{\top}\right)^{\top}, \mathbf{X}=\left(\mathbf{X}_{1}^{\top} \ldots \mathbf{X}_{k}^{\top}\right)^{\top}, \mathbf{Z}=\operatorname{diag}\left(\mathbf{Z}_{1}, \ldots, \mathbf{Z}_{k}\right)$, 
Figura 1.2 Gráfico de perfis individuais e médio para o diâmetro sistólico da aorta por unidade de peso $(\mathrm{mm} / \mathrm{kg})$ para o grupo de 32 recém-nascidos pré-termo PIG.

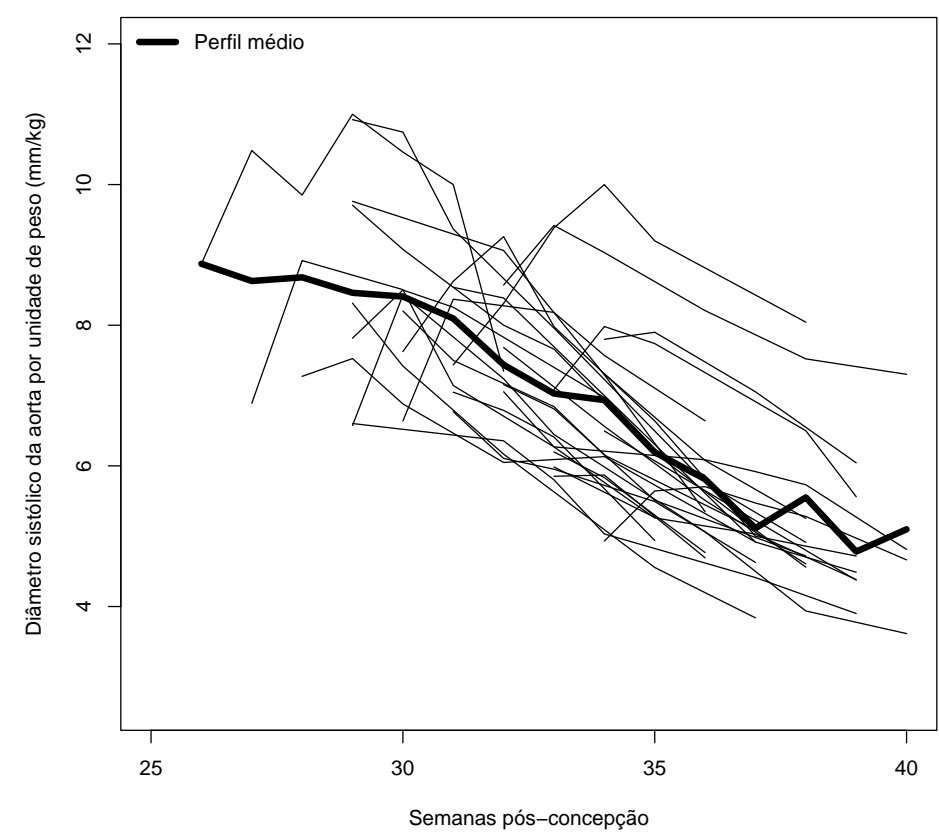

$\mathbf{b}=\left(\mathbf{b}_{1}^{\top}, \ldots, \mathbf{b}_{k}^{\top}\right)^{\top}$ e $\mathbf{e}=\left(\mathbf{e}_{1}^{\top}, \ldots, \mathbf{e}_{k}^{\top}\right)^{\top}$, podemos reescrever o modelo (1.1) compactamente como

$$
\mathbf{Y}=\mathbf{X} \boldsymbol{\beta}+\mathbf{Z b}+\mathbf{e}
$$

Em geral, assume-se que $\mathbb{E}[\mathbf{b}]=\mathbf{0}$ e $\mathbb{E}[\mathbf{e}]=\mathbf{0}$, com

$$
\operatorname{Cov}\left[\begin{array}{l}
\mathbf{b} \\
\mathbf{e}
\end{array}\right]=\left[\begin{array}{cc}
\boldsymbol{\Delta} & \mathbf{0}_{k q \times n} \\
\mathbf{0}_{n \times k q} & \boldsymbol{\Sigma}
\end{array}\right],
$$

em que $\mathbf{0}_{k_{1} \times k_{2}}$ representa uma matriz de dimensão $k_{1} \times k_{2}$ com todos elementos iguais a zero, $\boldsymbol{\Delta}$ e $\boldsymbol{\Sigma}$ são matrizes quadradas de ordens $k q$ e $n=\sum_{i=1}^{k} n_{i}$, positivas definidas, que correspondem, respectivamente, às matrizes de covariâncias dos vetores aleatórios $\mathbf{b}$ e $\mathbf{e}$. Em (1.2), os efeitos fixos são utilizados para modelar o valor esperado da variável resposta $\mathbf{Y}$, enquanto os efeitos aleatórios são utilizados para modelar sua estrutura de covariância. É comum assumir que $\mathbf{b}_{1}, \ldots, \mathbf{b}_{k} \stackrel{\text { iid }}{\sim} \mathcal{N}_{q}(\mathbf{0}, \boldsymbol{\Omega})$ (independentes e identicamente distribuídos) e $\mathbf{e}_{i} \sim \mathcal{N}_{n_{i}}\left(\mathbf{0}, \boldsymbol{\Sigma}_{i}\right)(i=1, \ldots, k)$, independentes. Então, $\boldsymbol{\Delta}=\mathbf{I}_{k} \otimes \boldsymbol{\Omega}$, com $\mathbf{I}_{k}$ representando a matriz identidade de ordem $k$ e $\mathbb{\otimes}$, o produto de Kronecker. Fazendo $\mathbf{w}=\mathbf{Z b}+\mathbf{e}$, obtém-se

$$
\mathbf{Y}=\mathbf{X} \boldsymbol{\beta}+\mathbf{w}
$$

Nobre, Juvêncio S. 
e essas especificações implicam que $\mathbf{w}$ tem distribuição normal $n$-variada com vetor de médias $\mathbf{0}_{n}$ e matriz de covariâncias

$$
\mathbf{V}=\mathbf{Z} \Delta \mathbf{Z}^{\top}+\mathbf{\Sigma}
$$

Em geral, supõe-se que $\boldsymbol{\Omega}$ e $\boldsymbol{\Sigma}$ são funções de poucos parâmetros (desconhecidos), $\boldsymbol{\theta}$, denominados parâmetros de covariância que funcionalmente independem dos parâmetros de localização $\boldsymbol{\beta}$.

O modelo (1.1) também pode ser intrepretado como um modelo linear em dois estágios (Laird e Ware, 1982). No primeiro estágio, condicionalmente ao conhecimento de $\mathbf{b}_{i}$ tem-se

$$
\mathbf{Y}_{i} \mid \mathbf{b}_{i} \sim \mathcal{N}_{n_{i}}\left(\mathbf{X}_{i} \boldsymbol{\beta}+\mathbf{Z}_{i} \mathbf{b}_{i}, \boldsymbol{\Sigma}_{i}\right), \quad i=1, \ldots, k
$$

No segundo estágio assume-se que

$$
\mathbf{b}_{i} \sim \mathcal{N}_{q}(\mathbf{0}, \mathbf{\Omega}), \quad i=1, \ldots, k
$$

Quando $\boldsymbol{\Sigma}_{i}$ é uma matriz diagonal, o modelo é denominado modelo de independência condicional, dado que as observações referentes a uma mesma unidade amostral são independentes, condicionalmente $\mathbf{a} \mathbf{b}_{i}$.

Vários métodos de estimação dos parâmetros do modelo (1.2) estão disponíveis na literatura; entre eles convém destacar os métodos de Máxima Verossimilhança (MV), Máxima Verossimilhança Restrita (MVR) e o método de Mínimos Quadrados (MQ), todos baseados no modelo marginal $\mathbf{Y} \sim \mathcal{N}_{n}(\mathbf{X} \boldsymbol{\beta}, \mathbf{V})$. Para mais detalhes, veja Laird e Ware (1982), Longford (1994), Pinheiro e Bates (2000), Verbeke e Molenberghs (2000), McCulloch e Searle (2001), Demidenko (2004), Lee et al. (2006) e Jiang (2007), por exemplo.

\subsection{Testes para componentes de variância}

No modelo (1.2) a estrutura de covariância intra-unidades amostrais é induzida pelos efeitos aleatórios. Nesse contexto, uma questão importante é a verificação da relevância da presença de determinados efeitos aleatórios, de forma a especificar, dentro desta classe de modelos, a estrutura "mais apropriada" para a matriz de covariâncias intra-unidades amostrais. Para exemplificar, considere o modelo (1.1) com $q=1$, ou seja, com apenas um efeito aleatório, nomeadamente, $b_{i 1} \sim \mathcal{N}\left(0, \sigma_{b}^{2}\right)$. O espaço paramétrico associado a $\sigma_{b}^{2}$ é $\left\{\sigma_{b}^{2} ; \sigma_{b}^{2} \geq 0\right\}=[0, \infty)$. Para avaliar a necessidade de inclusão desse efeito aleatório, podemos formular um teste da hipótese

$$
\mathcal{H}_{0}: \sigma_{b}^{2}=0
$$

Nobre, Juvêncio S. 
versus

$$
\mathcal{H}_{1}: \sigma_{b}^{2}>0
$$

Num contexto mais geral, com base no modelo (1.2) com $q>1$, suponha que desejamos verificar a necessidade de inclusão de um efeito aleatório, $b_{i(q+1)}$, dado que já foram incluídos $q$ efeitos aleatórios, digamos, $\mathbf{b}_{i}=\left(b_{i 1}, \ldots, b_{i q}\right)^{\top}$. Denotando $\operatorname{Var}\left(\mathbf{b}_{i}\right)=\mathbf{\Omega}_{1}, \operatorname{Var}\left(b_{i(q+1)}\right)=$ $\Omega_{2}, \operatorname{Cov}\left(\mathbf{b}_{i}, b_{i(q+1)}\right)=\Omega_{12} \mathrm{e}$

$$
\boldsymbol{\Omega}=\left[\begin{array}{cc}
\Omega_{1} & \Omega_{12} \\
\Omega_{12}^{\top} & \Omega_{2}
\end{array}\right]
$$

o espaço paramétrico referente aos parâmetros de covariância é $\{\operatorname{vech}(\Omega) \mid \Omega$ é não negativa definida (NND)\}. Então, o teste de interesse envolve a hipótese

$$
\mathcal{H}_{0}: \quad \boldsymbol{\Omega}=\left[\begin{array}{ll}
\boldsymbol{\Omega}_{1} & \mathbf{0} \\
\mathbf{0}^{\top} & 0
\end{array}\right], \text { com } \boldsymbol{\Omega}_{1} \text { positiva definida }(\mathrm{PD})
$$

versus

$$
\mathcal{H}_{1}: \Omega \text { NND, com } \Omega_{1} \text { PD e } \Omega_{2}>0
$$

Hipóteses do tipo (1.5) e (1.7) são denominadas não regulares, dado que a hipótese nula $\mathcal{H}_{0}$ define uma região situada na fronteira do espaço paramétrico. Como a teoria assintótica baseada na verossimilhança é obtida por meio de expansões em séries de Taylor da logverossimilhança em torno do verdadeiro valor do parâmetro (Cox e Hinkley, 1974, Cap.9; Feng e McCulloch, 1999; Sen e Singer, 1993, Cap.5 e Van der Vaart, 1998, Cap. 7) que assumimos ser um ponto interior do espaço paramétrico, em tais casos esta técnica não pode ser aplicada. Como conseqüência, a distribuição assintótica das estatísticas da razão de verossimilhanças generalizada, escore e Wald não é dada por uma distribuição qui-quadrado, como nos casos regulares. A obtenção da distribuição apropriada tem sido objeto de pesquisa de vários investigadores.

Self e Liang (1987) obtêm a distribuição assintótica da estatística da razão de verossimilhanças generalizada $\left(T_{R V G}\right)$ para variáveis aleatórias independentes e identicamente distribuídas (iid) em casos não regulares, e mostram que a distribuição assintótica nula é uma mistura de distribuições qui-quadrado centrais. Em particular, eles mostram que para testar (1.5) versus (1.6), a estatística $T_{R V G}$ segue uma distribuição assintótica (nula) $1 / 2 \chi_{0}^{2}+1 / 2 \chi_{1}^{2}$, que corresponde a uma mistura 50:50 de distribuições $\chi_{0}^{2}$ e $\chi_{1}^{2}$, com $\chi_{0}^{2}$ representando uma variável aleatória degenerada no ponto zero. Baseados nos resultados de Self e Liang (1987), 
Stram e Lee (1994) obtêm a distribuição assintótica da $T_{R V G}$ em várias situações de interesse. Por exemplo, para testar a inclusão de um novo efeito aleatório em um modelo que já inclui $q$ efeitos aleatórios, isto é, para testar (1.7) versus (1.8), eles mostraram que a distribuição assintótica da $T_{R V G}$ é $1 / 2 \chi_{q}^{2}+1 / 2 \chi_{q+1}^{2}$. Adicionalmente, supondo que este "novo" efeito aleatório é não correlacionado com os demais, ou seja, fazendo $\Omega_{12}=\mathbf{0}$ em (1.8), tal distribuição se reduz a $1 / 2 \chi_{0}^{2}+1 / 2 \chi_{1}^{2}$ (pois neste caso não há as $q$ restrições referentes às covariâncias). Um resultado análogo, obtido por meio de estudos de simulação, é descrito por Morrel (1998), que utiliza a estatística da razão de verossimilhanças restrita generalizada $\left(T_{R V R G}\right)$ ao invés da estatística $T_{R V G}$. Os pesos da mistura somente podem ser calculados analiticamente em casos especiais, dificultando assim a obtenção da distribuição assintótica da estatística $T_{R V G}$, principalmente quando se testa a inclusão de vários efeitos aleatórios simultaneamente. Além disso, o respectivo valor $p$ é obtido via simulação, para detalhes, veja por exemplo Silvapulle e Sen (2005).

Como observado em Giampaoli (1999) e em Giampaoli e Singer (2007), apenas em alguns estudos longitudinais com planejamentos balanceados os resultados obtidos em Self e Liang (1987) e (conseqüentemente) em Stram e Lee (1994) são válidos. Usando os resultados de Vu e Zhou (1997), que generalizaram os resultados de Self e Liang (1987) para situações em que as variáveis aleatórias não são iid, Giampaoli e Singer (2007) obtêm a distribuição assintótica da estatística $T_{R V G}$ e mostram que quando o interesse é avaliar a inclusão de um único efeito aleatório, dado que o modelo já inclui no máximo outro efeito aleatório, a distribuição correspondente coincide com aquela obtida por Stram e Lee (1994). Adicionalmente, os autores comentam sobre a dificuldade de se verificarem as condições de regularidade apresentadas em Vu e Zhou (1997) quando se aumenta a dimensão do vetor de efeitos aleatórios.

Silvapulle e Silvapulle (1995) propõem uma estatística do tipo escore $\left(T_{S}\right)$ para testar hipóteses não regulares unilaterais da forma $\mathcal{H}_{0}: \boldsymbol{\psi}=\mathbf{0}$ versus $\mathcal{H}_{1}: \boldsymbol{\psi} \geq \mathbf{0}$ (com pelo menos uma desigualdade estrita em $\left.\mathcal{H}_{1}\right)$, com $\boldsymbol{\theta}=\left(\boldsymbol{\psi}^{\top}, \rho^{\top}\right)^{\top}$ representando o vetor de parâmetros do modelo estatístico em questão. Eles provam que a distribuição assintótica nula de $T_{S}$ é uma mistura de distribuições qui-quadrado centrais, e que $T_{R V G}=T_{S}+o_{p}(1)$, ou seja, que as estatísticas $T_{R V G}$ e $T_{S}$ são assintoticamente equivalentes mesmo em situações não regulares. Silvapulle e Sen (2005, Cap.4) obtêm a distribuição assintótica das estatísticas do tipo escore e da razão de verossimilhanças generalizada para hipóteses não regulares mais gerais. Baseados no trabalho de Silvapulle e Silvapulle (1995), Verbeke e Molenberghs (2003) e Savalli et al. (2006) obtêm a distribuição assintótica da estatística do tipo escore para componentes de variância em modelos lineares mistos quando a distribuição conjunta de 
$\left(\mathbf{b}_{i}^{\top}, \mathbf{e}_{i}^{\top}\right)^{\top}$ é normal e elíptica, respectivamente. A vantagem dessa estatística com relação à estatística $T_{R V G}$, é que ela não requer que o modelo seja ajustado sob a hipótese alternativa. Todavia, assim como no teste da razão de verossimilhanças generalizada, a obtenção da distribuição de $T_{S}$ nos casos em que a dimensão do vetor de efeitos aleatórios é elevada é difícil.

Khuri et al. (1998) apresentam uma série de testes $F$ exatos para componentes de variância em vários casos particulares do modelo (1.2), como em modelos de ANOVA com efeitos aleatórios, baseados nas suposições de independência e normalidade dos vetores b e e. Os testes propostos, todavia, raramente são uniformemente mais poderosos (UMP). Eles apresentam testes ótimos na classe dos testes invariantes, como, testes invariantes UMP, melhor teste localmente invariante (locally best invariant), etc. Para detalhes sobre obtenção de testes invariantes, veja Schervish (1995), Khuri et al. (1998) ou Lehmman e Romano (2005), por exemplo.

Outras propostas são sugeridas na literatura. Por exemplo, Lin (1997) obtém um teste do tipo escore para verificar a nulidade conjunta de todas as componentes de variância em modelos lineares generalizados mistos usando uma aproximação de Laplace de segunda ordem da log-verossimilhança integrada (log-verossimilhança marginal). Na mesma linha do trabalho de Lin (1997), Zhu e Fung (2004) obtêm um teste do tipo escore para testar a inclusão de todos os candidatos a efeitos aleatórios em modelos semiparamétricos lineares mistos, sob a hipótese de normalidade apenas para o erro condicional. É importante salientar que ambos os testes são obtidos sob a abordagem marginal, ou seja, não levando em consideração o fato de que as componentes de variância devem ser não-negativas; além disso, esses autores assumem algumas condições de regularidade para os momentos centrais de terceira e quarta ordens dos efeitos aleatórios. Hall e Praestgaard (2001) consideram uma modificação do teste proposto por Lin (1997) obtida através de uma projeção do teste escore global nas direções que satisfazem a restrição $\operatorname{Var}\left(\mathbf{b}_{i}\right)=\Omega$ é não negativa definida. Eles provam que a distribuição assintótica nula é dada por uma mistura de distribuições qui-quadrado centrais e que além disso, o referido teste é mais poderoso do que o teste global proposto por Lin (1997). Novamente, a grande dificuldade reside na obtenção dos pesos da mistura. Andrews (2001) obtém, sob suposição de normalidade, um teste assintótico para verificar a significância de componentes de variância em modelos de coeficientes aleatórios. Todavia, a validade das condições de regularidade exigidas é de dificil verificação; sob a suposição de normalidade de $\mathbf{b}$ e e, Demidenko (2004, p.134-137) propõe um teste $F$ para avaliar a necessidade de inclusão de todos os candidatos a efeitos aleatórios conjuntamente, no modelo (1.2).

Todas as abordagens supracitadas são baseadas na verossimilhança e por isso são de 
difícil aplicação em situações gerais; elas são válidas, e.g., quando as distribuições dos efeitos aleatórios e dos erros intra-unidades amostrais são normais ou elípticas. O objetivo do presente trabalho é propor testes para componentes de variâncias baseados em decomposições de estatísticas $U$ que prescindem da especificação da forma das distribuições e exigem apenas a suposição de existência de alguns de seus momentos. Os testes propostos podem ser aplicados a uma classe muito mais ampla de modelos, que inclui os modelos lineares mistos elípticos, em que não necessariamente o efeito aleatório e o erro condicional possuem a mesma distribuição (por exemplo, o efeito aleatório com distribuição $t$ e erro condicional com distribuição normal), os modelos lineares mistos assimétricos, em que se assumem distribuições assimétricas para os efeitos aleatórios e/ou para o erro condicional, como em Ma et al. (2004) e Arellano-Valle et al. (2005).

No Capítulo 2 apresentamos noções e propriedades básicas das estatísticas $U$ utilizadas no desenvolvimento da tese. Tal classe de estatísticas teve sua gênese nos trabalhos de Halmos (1946) e Hoeffding (1948). Uma das grandes vantagens desta classe de estatísticas é a sua estrutura simples e o fato de não ser necessário especificar completamente o modelo probabilístico em questão para obtenção de boas propriedades inferenciais. No Capítulo 3 descrevemos os testes para componentes de variância em modelos lineares mistos. A decomposição utilizada é similar àquela empregada em ANOVA e MANOVA e já foi considerada em outras classes de modelos, como por exemplo, em Pinheiro et al. (2005), Schaid et al. (2005), Sen (2006) e Pinheiro et al. (2007). A vantagem desta abordagem, comparada com as abordagens baseadas na verossimilhança, é que ela não requer nenhum tipo de expansão em torno do verdadeiro valor do parâmetro, não sendo afetada assim pela possível localização do parâmetro na fronteira do espaço paramétrico. No capítulo 4 apresentamos dois exemplos práticos aos quais aplicamos os testes desenvolvidos. Finalmente, no Capítulo 5 apresentamos as conclusões deste trabalho e discutimos futuras pesquisas.

Nobre, Juvêncio S. 


\section{Capítulo 2}

\section{Estatísticas U}

Sejam $\Im$ uma classe de funções distribuição em $\mathbb{R}^{p}$ (por exemplo, de distribuições simétricas, com $r$-ésimo momento finito, etc.) e $\theta(F), F \in \Im$, um funcional com domínio em $\Im$, isto é,

$$
\theta: \Im \longmapsto \mathbb{R} \text {. }
$$

Concentraremos nossa atenção nos chamados funcionais estatísticos regulares de grau $m$, ou parâmetros estimáveis, no sentido denotado por Halmos(1946) e em Hoeffding (1948), para os quais existe uma função núcleo, de $m$ variáveis, $g$ tal que

$$
\theta(F)=\int \ldots \int g\left(x_{1}, \ldots, x_{m}\right) \prod_{i=1}^{m} d F\left(x_{i}\right),
$$

$\forall \Im \subseteq\{F ;|\theta(F)|<\infty\}$. Na literatura estatística, $\theta(F)$ também é comumente denominado funcional homogêneo de grau $m$ (Halmos, 1946 e Sen, 1981, por exemplo). Sem perda de generalidade, consideraremos $g$ simétrica (ou permutável), isto é, invariante sob permutações dos argumentos. Nos casos em que $g$ não é simétrica, basta considerar $g^{*}\left(x_{1}, \ldots, x_{m}\right)=$ $(m !)^{-1} \sum_{\wp\{1, \ldots, m\}} g\left(x_{i_{1}}, \ldots, x_{i_{m}}\right)$, em que a soma é estendida a todas as $m$ ! permutações dos indíces $(1, \ldots, m)$, no lugar de $g\left(x_{1}, \ldots, x_{m}\right)$.

Como exemplo, considere o caso em que $m=2$ e $g\left(x_{1}, x_{2}\right)=\left(x_{1}-x_{2}\right)^{2} / 2$; então $\theta(F)=$ $\mathbb{E}\left[\left(X_{1}-X_{2}\right)^{2} / 2\right]=\operatorname{Var}\left[X_{1}\right]=\sigma^{2}$ com $\Im$ denotando a classe de todas as distribuições com segundo momento finito. Existem funcionais $\theta(F)$ que não podem ser expressos da forma (2.1), para $m$ fixo. Um exemplo é o funcional quantílico, $\theta(F)=F^{-1}(p)=\inf \{x ; F(x) \geq$ $p\}, \quad p \in(0,1), \quad F \in \Im$, com $\Im$ representando a classe de todas as funções distribuição que admitem um único quantil $F^{-1}(p), p \in(0,1)$.

Suponha que o interesse consiste na estimação de $\theta$, dado em (2.1), com base em uma amostra aleatória $X_{1}, \ldots, X_{n}$ de uma particular distribuição $F \in \Im$. Podemos considerar o seguinte estimador não viesado para $\theta(F)$ :

$$
U_{n}=\left(\begin{array}{c}
n \\
m
\end{array}\right)^{-1} \sum_{1 \leq i_{1}<i_{2}<\ldots<i_{m} \leq n} g\left(X_{i_{1}}, \ldots X_{i_{m}}\right)=\left(\begin{array}{c}
n \\
m
\end{array}\right)^{-1} \sum_{(n, m)} g\left(X_{i_{1}}, \ldots X_{i_{m}}\right),
$$


em que $\sum_{(n, m)}$ indica que a soma é estendida sob todas as $\left(\begin{array}{c}n \\ m\end{array}\right)$ combinações de $m$ elementos $\left\{i_{1}, \ldots, i_{m}\right\}$ de $\{1, \ldots, n\}$. Este tipo de estimador é denominado estatística $\boldsymbol{U}$ (U statistic) de grau $m$. A estatística $U_{n}$ definida acima é uma função mensurável das estatísticas de ordem (pois $U_{n}$ é uma função simétrica/permutável das observações), especificamente $U_{n}=$ $\mathbb{E}\left[g\left(X_{1}, \ldots, X_{m}\right) \mid X_{(1)}, \ldots, X_{(n)}\right]$. Como o vetor das estatísticas de ordem $\left(X_{(1)}, \ldots, X_{(n)}\right)^{\top}$ é completo para a classe $\Im$ na maioria dos casos de interesse [veja por exemplo, Halmos (1946), Fraser (1954) e Bell et al. (1960)], então pelo Teorema de Rao-Blackwell, $U_{n}$ é o estimador não viesado de variância uniformemente mínima (ENVVUM) de $\theta$, e de uma forma mais geral, ele também é o estimador de risco uniformemente mínimo, para qualquer função de perda convexa (veja por exemplo, Lehmann e Casella, 1998, p.47). Propriedades adicionais de estatísticas $U$, tais como sua caracterização como um processo martingal (clássico e reverso), propriedades de invariância, leis dos grandes números, sua relação com o funcional de Von Mises e expansões de Edgeworth podem ser encontradas em Hoeffding (1948), Hoeffding (1961), Berk (1966), Serfling (1980), Sen (1981), Van Zwet (1984), Lee (1990), Sen e Singer (1993), Lehmann (1998) e Chow e Teicher (2003), por exemplo.

Quando o interesse reside na estimação da variância $\sigma^{2}$, i.e., quando $\theta(F)=\sigma^{2}$, a estatística $U$ em questão é dada por

$$
\hat{\sigma}_{n}^{2}=\left(\begin{array}{l}
n \\
2
\end{array}\right)^{-1} \sum_{1 \leq i<j \leq n} \frac{\left(X_{i}-X_{j}\right)^{2}}{2}=(n-1)^{-1} \sum_{i=1}^{n}\left(X_{i}-\bar{X}\right)^{2}=S_{n}^{2},
$$

que é a variância amostral.

Definindo,

$$
\begin{aligned}
g_{c}\left(x_{1}, \ldots, x_{c}\right) & =\mathbb{E}\left[g\left(x_{1}, \ldots, x_{c}, X_{c+1}, \ldots, X_{m}\right)\right] \\
& =\mathbb{E}\left[g\left(X_{1}, \ldots, X_{m}\right) \mid X_{1}=x_{1}, \ldots, X_{c}=x_{c}\right]
\end{aligned}
$$

para $c=1,2, \ldots, m$, e denotando as respectivas variâncias por

$$
\varsigma_{c}^{2}=\operatorname{Var}\left[g_{c}\left(X_{1}, \ldots, X_{c}\right)\right]
$$

com $\varsigma_{0}^{2}=0$, pode-se mostrar (veja Lee, 1990, p.12-13, por exemplo) que a variância de $U_{n}$, definida em (2.2), é dada por

$$
\operatorname{Var}\left[U_{n}\right]=\left(\begin{array}{c}
n \\
m
\end{array}\right)^{-1} \sum_{c=1}^{m}\left(\begin{array}{c}
m \\
c
\end{array}\right)\left(\begin{array}{c}
n-m \\
m-c
\end{array}\right) \varsigma_{c}^{2} .
$$

Como $g_{m}=g$ e $\varsigma_{c}^{2}$ é crescente em $c$ (veja Hoeffding, 1948), uma condição necessária e suficiente para que $\operatorname{Var}\left[U_{n}\right]$ seja finita, é que $\varsigma_{m}^{2}=\operatorname{Var}\left[g\left(X_{1}, \ldots, X_{m}\right)\right]<\infty$. 
Considerando novamente o interesse na estimação de $\theta(F)=\sigma^{2}$, temos

$$
g_{1}(x)=\mathbb{E}\left[\left(x-X_{2}\right)^{2} / 2\right]=\frac{\left(\sigma^{2}+(x-\mu)^{2}\right)}{2}
$$

com $\operatorname{Var}\left[g_{1}(X)\right]=\varsigma_{1}^{2}=\left(\mu_{4}-\sigma^{4}\right) / 4, \mu_{4}$ representando o quarto momento central de $X$ e $\mu=\mathbb{E}[X]$. É possível mostrar também que

$$
\varsigma_{2}^{2}=\operatorname{Var}\left[g_{2}\left(X_{1}, X_{2}\right)\right]=\operatorname{Var}\left[\left(X_{1}-X_{2}\right)^{2} / 2\right]=\frac{\left(\mu_{4}+\sigma^{4}\right)}{2} .
$$

E em função de (2.5)

$$
\operatorname{Var}\left[S_{n}^{2}\right]=\left(\begin{array}{l}
n \\
2
\end{array}\right)^{-1}\left\{2(n-2) \varsigma_{1}^{2}+\varsigma_{2}^{2}\right\}=\frac{\mu_{4}}{n}-\frac{(n-3) \sigma^{4}}{n(n-1)}
$$

Sob a suposição de normalidade, $\mu_{4}=3 \sigma^{4}$, que pela identidade acima implica que $\operatorname{Var}\left(S_{n}^{2}\right)=$ $2 \sigma^{4} /(n-1)$.

De (2.5), temos que $\operatorname{Var}\left[U_{n}\right]=O\left(n^{-1}\right)$ se $\varsigma_{1}^{2}>0$, e no caso em que a estatística $U$ é estacionária de ordem $d$ (i.e., quando $\left.0=\varsigma_{1}^{2}=\varsigma_{2}^{2}=\ldots=\varsigma_{d}^{2}<\varsigma_{d+1}^{2}\right)$, então $\operatorname{Var}\left[U_{n}\right]=$ $O\left(n^{-(d+1)}\right)$. Além disso, é possível mostrar (Hoeffding, 1948) que $\operatorname{Var}\left[\sqrt{n} U_{n}\right]$ é uma função decrescente de $n$, de tal forma que

$$
\operatorname{Var}\left[U_{n}\right]=\frac{m^{2} \varsigma_{1}^{2}}{n}+O\left(n^{-2}\right)
$$

Nos casos em que $m \geq 2$, os termos da soma (2.2) não são todos independentes, o que não permite aplicar diretamente o Teorema Limite Central (TLC) clássico com a finalidade de se obter a distribuição assintótica de $U_{n}$. Uma alternativa consiste em explorar a estrutura de martingal (ou martingal reverso) associada ao processo $\left\{U_{n}\right\}_{n \geq m}$ (veja, Serfling, 1980, Sen, 1981 e Sen e Singer, 1993, por exemplo) e usar o TLC para martingais (ou para martingais reversos); todavia, nesse contexto, nem sempre é fácil verificar as condições de regularidade, principalmente em situações em que a estatística é estacionária. Hoeffding (1948) obteve a distribuição assintótica de $U_{n}$ no caso em que $\varsigma_{1}^{2}>0$ por meio de uma projeção de $U_{n}$ em relação a $X_{1}, \ldots, X_{n}$, resultando em uma soma de variáveis aleatórias independentes e identicamente distribuídas mais uma parcela assintoticamente desprezável (em probabilidade). Para detalhes, veja, por exemplo, Hoeffding (1948), Lee (1990) ou Sen e Singer (1993, p. 212). Isto permite utilizar o TLC clássico juntamente com o Teorema de Slutsky de forma a obter a seguinte distribuição assintótica

$$
\sqrt{n}\left(U_{n}-\theta\right) \stackrel{\mathcal{D}}{\longrightarrow} \mathcal{N}\left(0, m^{2} \varsigma_{1}^{2}\right) .
$$

A decomposição $\boldsymbol{H}$ de estatísticas $U$, desenvolvida por Hoeffding (1961), é bastante 
útil para obter propriedades assintóticas de $U_{n}$, principalmente nos casos em que ela é estacionária. Essa abordagem consiste em representar uma estatística $U$ de grau $m$ como uma soma de $m$ estatísticas $U$ não-correlacionadas de graus $1,2, \ldots, m$, com variâncias de ordens $n^{-1}, \ldots, n^{-m}$, respectivamente. Se $U_{n}$ é uma estatística $U$ de grau $m, \operatorname{com} \mathbb{E}\left[U_{n}\right]=\theta$, então a correspondente decomposição $H$ é

$$
U_{n}=\theta+\sum_{j=1}^{m}\left(\begin{array}{c}
m \\
j
\end{array}\right) H_{n}^{(j)}
$$

em que

$$
H_{n}^{(j)}=\left(\begin{array}{c}
n \\
j
\end{array}\right)^{-1} \sum_{(n, j)} h^{(j)}\left(X_{v_{1}}, \ldots, X_{v_{j}}\right)
$$

com a função $h^{(j)}\left(x_{i_{1}}, \ldots, x_{i_{j}}\right)$ definida como

$$
\begin{aligned}
h^{(1)}\left(x_{1}\right) & =g_{1}\left(x_{1}\right)-\theta \\
h^{(c)}\left(x_{1}, \ldots, x_{c}\right) & =g_{c}\left(x_{1}, \ldots, x_{c}\right)-\sum_{j=1}^{c-1} \sum_{(c, j)} h^{(j)}\left(x_{i_{1}}, \ldots, x_{i_{j}}\right)-\theta, \quad c=2, \ldots, m,
\end{aligned}
$$

de forma que $\operatorname{Cov}\left(H_{n}^{(j)}, H_{n}^{\left(j^{\prime}\right)}\right)=0, \forall j \neq j^{\prime}$; para detalhes, veja Lee (1990), por exemplo. Denotando $R_{n}^{(c)}$ o resto obtido por meio do truncamento de (2.7) no c-ésimo termo, temos

$$
U_{n}=\theta+\sum_{j=1}^{c}\left(\begin{array}{c}
m \\
j
\end{array}\right) H_{n}^{(j)}+R_{n}^{(c)},
$$

com $\mathbb{E}\left[R_{n}^{(c)}\right]=0$ e $\operatorname{Var}\left[R_{n}^{(c)}\right]=O\left(n^{-(c+1)}\right)$. Por exemplo, no caso em que $\varsigma_{1}^{2}>0$, pode-se usar o fato de que

$$
U_{n}-\theta=m H_{n}^{(1)}+R_{n}^{(1)}
$$

com $H_{n}^{(1)}=n^{-1} \sum_{j=1}^{n}\left(g_{1}\left(X_{j}\right)-\theta\right)$ e $R_{n}^{(1)}$ tal que $\operatorname{Var}\left[R_{n}^{(1)}\right]=O\left(n^{-2}\right)$. Notemos que $H_{n}^{(1)}$ é uma média de $n$ variáveis aleatórias iid de média 0 (pois $\mathbb{E}\left[g_{1}\left(X_{j}\right)\right]=\theta$ ) e variância $\varsigma_{1}^{2}$, e como $\mathbb{E}\left[R_{n}^{(1)}\right]=0 \mathrm{com} \sqrt{n} \operatorname{Var}\left[R_{n}^{(1)}\right]=O\left(n^{-1}\right)$ o que implica $\sqrt{n} R_{n}^{(1)}=o_{p}(1)$. Então, usando o TLC clássico e o Teorema de Slutsky, obtemos (2.6). Como ilustração, consideremos novamente a situação em que $\theta(F)=\sigma^{2}$. Como $g_{1}(x)=\left\{\sigma^{2}+(x-\mu)^{2}\right\} / 2$ e $g_{2}\left(x_{1}, x_{2}\right)=g\left(x_{1}, x_{2}\right)=$ $\left(x_{1}-x_{2}\right)^{2} / 2$, temos

$$
h^{(1)}\left(x_{1}\right)=g_{1}\left(x_{1}\right)-\sigma^{2}=\left\{\left(x_{1}-\mu\right)^{2}-\sigma^{2}\right\} / 2
$$


e

$$
\begin{aligned}
h^{(2)}\left(x_{1}, x_{2}\right) & =g_{2}\left(x_{1}, x_{2}\right)-\left(g_{1}\left(x_{1}\right)-\sigma^{2}\right)-\left(g_{1}\left(x_{2}\right)-\sigma^{2}\right)-\sigma^{2} \\
& =\left(x_{1}-x_{2}\right)^{2} / 2-\left\{\left(x_{1}-\mu\right)^{2}+\left(x_{2}-\mu\right)^{2}\right\} / 2=-\left(x_{1}-\mu\right)\left(x_{2}-\mu\right) .
\end{aligned}
$$

Portanto, por (2.7), a decomposição $H$ de $S_{n}^{2}$ é dada por $S_{n}^{2}=\sigma^{2}+2 H_{n}^{(1)}+H_{n}^{(2)}$, em que

$$
\begin{aligned}
& H_{n}^{(1)}=n^{-1} \sum_{i=1}^{n} \frac{\left(X_{i}-\mu\right)^{2}-\sigma^{2}}{2} \\
& H_{n}^{(2)}=-\left(\begin{array}{l}
n \\
2
\end{array}\right)^{-1} \sum_{1 \leq i<j \leq n}\left(X_{i}-\mu\right)\left(X_{j}-\mu\right) .
\end{aligned}
$$

Além disso, por (2.6), obtemos

$$
\sqrt{n}\left(S_{n}^{2}-\sigma^{2}\right) \stackrel{\mathcal{D}}{\longrightarrow} \mathcal{N}\left(0, \mu_{4}-\sigma^{4}\right)
$$

A convergência para a distribuição normal, nunca é válida quando a estatística $U_{n}$ é estacionária de ordem d. Serfling (1980, p.193-194) obtém a distribuição assintótica de $n\left(U_{n}-\theta\right)$ para o caso em que $U_{n}$ é estacionária de ordem 1 . Para os casos em que $d \geq 2$, veja Lee (1990, cap.3), por exemplo. Lee (1990, p.34-35) apresenta uma interpretação geométrica da decomposição $H$, e mostra sua conexão com decomposições bem conhecidas, como aquela utilizada em ANOVA.

Muitas generalizações do que foi aqui apresentado podem ser consideradas; por exemplo:

i) permitir que o funcional $\theta$ seja função de várias classes de função de distribuição $\Im_{1}, \ldots, \Im_{t}$

ii) relaxar a suposição de que as variáveis $X_{1}, \ldots, X_{n}$ são iid;

iii) considerar que a amostra é retirada de uma população finita;

iv) considerar estatísticas $U$ ponderadas (muito útil quando se tem dados omissos), etc;

Serfling (1980), Sen (1981) e Lee (1990) apresentam as propriedades destas possíveis generalizações além de uma extensa lista de referências versando sobre outras não abordadas aqui.

No presente texto, trataremos apenas do item i), ou seja, situações em que $\theta$ é um funcional regular de várias classes de funções distribuição, $\Im_{1}, \ldots, \Im_{t}$, isto é,

$$
\theta:\left(\Im_{1}, \ldots, \Im_{t}\right) \longmapsto \mathbb{R}
$$

Nobre, Juvêncio S. 
com

$$
\theta(F)=\int \ldots \int g\left(x_{11}, \ldots, x_{1 m_{1}}, \ldots, x_{t_{1}}, \ldots, x_{t_{m t}}\right) \prod_{i=1}^{m_{1}} d F_{1}\left(x_{1_{i}}\right) \ldots \prod_{i=1}^{m_{t}} d F_{t}\left(x_{t_{i}}\right),
$$

em que $g$ representa uma função de $\sum_{i=1}^{t} m_{i}$ variáveis, simétrica em cada subconjunto $\left\{x_{j_{1}}, \ldots, x_{j_{m_{j}}}\right\}$. Assim como no caso anterior, o interesse reside na estimação de $\theta$ com base em amostras de tamanho $n_{j}$ de variáveis aleatórias iid, digamos $X_{j_{1}}^{j}, \ldots, X_{j_{n_{j}}}^{j}$, com funções distribuição $F_{j} \in \Im_{j}$ com independência entre observações de classes distintas. Na prática, usualmente estas classes representam estratos populacionais.

Usando uma clara extensão de (2.2), podemos definir uma estatística $U$ generalizada de grau $\left(m_{1}, \ldots, m_{t}\right)$ como

$$
U_{n}=\prod_{i=1}^{t}\left(\begin{array}{c}
n_{i} \\
m_{i}
\end{array}\right)^{-1} \sum_{\left(n_{1}, m_{1}\right)} \ldots \sum_{\left(n_{t}, m_{t}\right)} g\left(X_{i_{1}}^{1}, \ldots X_{i_{m_{1}}}^{1}, \ldots X_{i_{1}}^{t}, \ldots, X_{i_{m_{t}}}^{t}\right) .
$$

Características e propriedades de estatísticas $U$ generalizadas, tais como variância, decomposição $H$, distribuição assintótica, etc. seguem o mesmo padrão previamente apresentado; para detalhes, veja Serfling (1980, cap.5), Sen (1981, cap.3) e Lee (1990, cap.2), por exemplo.

Recentemente, muitas aplicações fazem uso desta teoria, bem como de decomposições de estatísticas $U$ para propor testes em modelos complexos. Podemos citar, por exemplo, Kowalski (2001), Kowalski et al. (2002), Tzeng et al. (2003), Pinheiro et al. (2005), Schaid et al. (2005), Bertail e Tressou (2006), Sen (2006) e Pinheiro et al. (2007). Para mais aplicações, veja por exemplo, Lee (1990, Cap.6).

Nobre, Juvêncio S. 


\section{Capítulo 3}

\section{Testes para componentes de variância}

\subsection{Introdução}

Neste capítulo obtemos testes assintóticos para componentes de variância em modelos lineares mistos baseados em decomposições de estatísticas $U$. Na Seção 3.2 apresentamos o teste obtido para modelos de ANOVA com um único fator aleatório; na Seção 3.3 estendemos o teste para modelos em que a média da variável resposta é modelada por covariáveis, e na Seção 3.4 consideramos um modelo de retas com coeficientes aleatórios e apresentamos um teste para a hipótese de que a componente de variância da inclinação aleatória é nula na presença do intercepto aleatório.

Em modelos com dois ou mais efeitos aleatórios, as estatísticas de teste apresentados nas Seções 3.2 e 3.3 também podem ser utilizadas para hipóteses de que as variâncias dos efeitos aleatórios são simultaneamente nulas. Testes exatos para verificar a inclusão simultânea de todos os candidatos a efeitos aleatórios existem apenas sob a hipótese de normalidade e independência dos vetores de erros condicionais e dos efeitos aleatórios. Os testes propostos aqui prescindem da suposição de normalidade dos componentes aleatórios do modelo. O teste apresentado na Seção 3.4 também pode ser utilizado na situação em que temos interesse em verificar se duas ou mais componentes de variância são simultaneamente nulas, dado que já foram incluídos $q$ efeitos aleatórios no modelo.

\subsection{Modelo de ANOVA com um único efeito aleatório}

Consideremos o modelo

$$
Y_{i j}=\mu+b_{i}+e_{i j}, \quad i=1, \ldots, k, \quad j=1, \ldots, n_{i}
$$

em que para $i=1, \ldots, k, j=1, \ldots, n_{i}$

$$
b_{i} \stackrel{\mathrm{iid}}{\sim}\left(0, \sigma_{b}^{2}\right)
$$




$$
\begin{aligned}
& e_{i j} \stackrel{\text { iid }}{\sim}\left(0, \sigma_{e}^{2}\right) \text { com } \mathbb{E}\left[e_{i j}^{4}\right]=\mu_{4 e}<\infty \\
& b_{i} \text { e } e_{i j} \text { independentes. }
\end{aligned}
$$

Vamos supor que o interesse seja verificar a necessidade de inclusão do efeito aleatório, ou seja, testar

$$
\mathcal{H}_{0}: \sigma_{b}^{2}=0 \text { versus } \mathcal{H}_{1}: \sigma_{b}^{2}>0
$$

Nesta seção, apresentamos um teste assintótico para (3.5) sob o modelo (3.1). É válido ressaltar, que o procedimento desenvolvido para testar a inexistência dos efeitos aleatórios, também é válido na situação em que as variâncias dos efeitos aleatórios são distintas, i.e., quando $b_{i} \stackrel{\text { ind }}{\sim}\left(0, \sigma_{i}^{2}\right)$.

A teoria assintótica geralmente é aplicada a casos em que o número total de observações é "grande". Em modelos de medidas repetidas do tipo (3.1), é natural considerar que o número de unidades amostrais $(k)$ cresce mas o número de observações por unidades amostrais $\left(n_{i}\right)$ se mantém limitado, como consideram, por exemplo, Self e Liang (1987). Quando se tem interesse em verificar a significância de uma única componente de variância, Crainiceanu e Ruppert (2004) mostram que, sob suposição de normalidade, a distribuição assintótica nula da estatística $T_{R V G}$, e conseqüentemente da estatística $T_{S}$, não é adequada em situações em que se tem poucas unidades amostrais e muitas observações por unidade amostral. Eles obtêm a distribuição assintótica nula de $T_{R V G}$ e $T_{R V R G}$ nessas situações, além de apresentar um algoritmo para simular a sua distribuição nula para amostras de tamanho moderado. Todavia, a complexidade de sua proposta cresce com o número de unidades amostrais, dificultando assim sua implementação.

Aqui, consideramos o caso em que $k$ é "grande" e $n_{i}$ é "limitado" (fixado), mas o teste proposto também é válido nas situações em que $k$ é "limitado" e $n_{i}$ é "grande" (veja Apêndice B). Akritas e Arnold (2000) obtêm a distribuição assintótica (quando $k \rightarrow \infty$ ) de um teste do tipo $F$ para testar (3.5) nesta situação. O teste é obtido sob certas condições de regularidade que incluem a existência de mais do que os quatro primeiros momentos da distribuição do erro condicional, e exigem que o terceiro momento de $e_{i j}$ seja nulo, etc. Além disso, o procedimento desenvolvido para obter a distribuição da estatística de teste é diferente para estudos balanceados e desbalanceados.

Definindo $g\left(Y_{i j}, Y_{i j^{\prime}}\right)=\left(Y_{i j}-Y_{i j^{\prime}}\right)^{2} / 2$, temos $\mathbb{E}\left[g\left(Y_{i j}, Y_{i j^{\prime}}\right)\right]=\mathbb{E}\left[\left(e_{i j}-e_{i j^{\prime}}\right)^{2}\right] / 2=\sigma_{e}^{2}$. Logo, um estimador não viesado de $\sigma_{e}^{2}$, baseado somente nas $n_{i}$ observações da $i$-ésima unidade 
amostral, é dado pela seguinte estatística $U$

$$
\begin{aligned}
U_{i} & =\left(\begin{array}{c}
n_{i} \\
2
\end{array}\right)^{-1} \sum_{1 \leq j<j^{\prime} \leq n_{i}} g\left(Y_{i j}, Y_{i j^{\prime}}\right)=\left(\begin{array}{c}
n_{i} \\
2
\end{array}\right)^{-1} \sum_{1 \leq j<j^{\prime} \leq n_{i}}\left(Y_{i j}-Y_{i j^{\prime}}\right)^{2} / 2 \\
& =\left(n_{i}-1\right)^{-1} \sum_{j=1}^{n_{i}}\left(Y_{i j}-\bar{Y}_{i .}\right)^{2}=S_{i}^{2}, \quad i=1, \ldots, k .
\end{aligned}
$$

Por outro lado, como

$$
\left(Y_{i j}-Y_{i^{\prime} j^{\prime}}\right)^{2}=\left\{\left(b_{i}-b_{i^{\prime}}\right)^{2}+\left(e_{i j}-e_{i^{\prime} j^{\prime}}\right)^{2}+2\left(b_{i}-b_{i^{\prime}}\right)\left(e_{i j}-e_{i^{\prime} j^{\prime}}\right)\right\}
$$

e $\mathbb{E}\left[\left(b_{i}-b_{i^{\prime}}\right)\left(e_{i j}-e_{i^{\prime} j^{\prime}}\right)\right]=0$, concluímos que $\mathbb{E}\left[g\left(Y_{i j}, Y_{i^{\prime} j^{\prime}}\right)\right]=\left\{2 \sigma_{b}^{2}+2 \sigma_{e}^{2}\right\} / 2=\sigma_{b}^{2}+\sigma_{e}^{2}$. Portanto um estimador não viesado de $\sigma_{b}^{2}+\sigma_{e}^{2}$, baseado apenas nas observações referentes às unidades amostrais $i$ e $i^{\prime}$ é dado pela seguinte estatística $U$ generalizada de ordem $(1,1)$

$$
U_{i i^{\prime}}=\left(n_{i} n_{i^{\prime}}\right)^{-1} \sum_{j=1}^{n_{i}} \sum_{j^{\prime}=1}^{n_{i^{\prime}}} \frac{\left(Y_{i j}-Y_{i^{\prime} j^{\prime}}\right)^{2}}{2}, \quad 1 \leq i<i^{\prime} \leq k .
$$

Denotando o número total de observações por $n=\sum_{i=1}^{k} n_{i}$, podemos ordenar as observações lexicograficamente

$$
Y_{11}, \ldots, Y_{1 n_{1}}, Y_{21}, \ldots, Y_{2 n_{2}}, \ldots, Y_{k 1}, \ldots, Y_{k n_{k}}
$$

e fazer $Y_{1}=Y_{11}, Y_{2}=Y_{12}, \ldots, Y_{n_{1}}=Y_{1 n_{1}}, Y_{n_{1}+1}=Y_{21}, \ldots, Y_{n}=Y_{k n_{k}}$, de forma que (3.8) possa ser representado por

$$
Y_{1}, \ldots, Y_{n_{1}}, Y_{n_{1}+1}, \ldots, Y_{n_{1}+n_{2}}, \ldots, Y_{n_{1}+\ldots+n_{k-1}+1}, \ldots, Y_{n}
$$

com as $n_{1}$ primeiras observações correspondendo àquelas da unidade amostral 1, as próximas $n_{2}$ observações correspondendo àquelas da unidade amostral 2, e assim por diante. O ENVVUM da variância das observações é dado pela seguinte estatística $U$

$$
U_{n}^{0}=\left(\begin{array}{l}
n \\
2
\end{array}\right)^{-1} \sum_{1 \leq r<s \leq n} \frac{1}{2}\left(Y_{r}-Y_{s}\right)^{2}
$$

Nossa proposta é utilizar uma idéia similar àquela usada em ANOVA, ou seja, decompor $U_{n}^{0}$ de uma maneira conveniente, de forma a obter uma estatística de teste para a hipótese de interesse. Como

$$
\begin{aligned}
\sum_{1 \leq r<s \leq n_{1}} \frac{1}{2}\left(Y_{r}-Y_{s}\right)^{2} & =\left(\begin{array}{c}
n_{1} \\
2
\end{array}\right) U_{1} \\
\sum_{\substack{r \in\left\{1, \ldots, n_{1}\right\} \\
s \in\left\{n_{1}+1, \ldots, n_{1}+n_{2}\right\}}} \frac{1}{2}\left(Y_{r}-Y_{s}\right)^{2} & =n_{1} n_{2} U_{12}
\end{aligned}
$$

Nobre, Juvêncio S. 
e seguindo a mesma linha, podemos reescrever a estatística $U_{n}^{0}$ como

$$
\begin{aligned}
U_{n}^{0} & =\left(\begin{array}{c}
n \\
2
\end{array}\right)^{-1}\left\{\sum_{i=1}^{k}\left(\begin{array}{c}
n_{i} \\
2
\end{array}\right) U_{i}+\sum_{1 \leq i<i^{\prime} \leq k} n_{i} n_{i^{\prime}} U_{i i^{\prime}}\right\} \\
& =\sum_{i=1}^{k} \frac{n_{i}\left(n_{i}-1\right)}{n(n-1)} U_{i}+2 \sum_{1 \leq i<i^{\prime} \leq k} \frac{n_{i} n_{i^{\prime}}}{n(n-1)} U_{i i^{\prime}}
\end{aligned}
$$

que é uma combinação linear de estatísticas $U$. O primeiro e o segundo termos de (3.9) correspondem aos componentes intra e inter unidades amostrais, respectivamente. Dado que

$$
\frac{n_{i}\left(n_{i}-1\right)}{n(n-1)}=\frac{n_{i}}{n}-\frac{n_{i}\left(n-n_{i}\right)}{n(n-1)}
$$

o primeiro termo de (3.9) pode ser reescrito como

$$
\sum_{i=1}^{k} \frac{n_{i}\left(n_{i}-1\right)}{n(n-1)} U_{i}=\sum_{i=1}^{k} \frac{n_{i}}{n} U_{i}-\sum_{i=1}^{k} \frac{n_{i}\left(n-n_{i}\right)}{n(n-1)} U_{i}
$$

e lembrando que $\sum_{i^{\prime} \neq i} n_{i^{\prime}}=n-n_{i}$, obtemos

$$
\begin{aligned}
\sum_{i=1}^{k} \frac{n_{i}\left(n_{i}-1\right)}{n(n-1)} U_{i} & =\sum_{i=1}^{k} \frac{n_{i}}{n} U_{i}-\sum_{i=1}^{k} \sum_{i^{\prime} \neq i} \frac{n_{i} n_{i^{\prime}}}{n(n-1)} U_{i} \\
& =\sum_{i=1}^{k} \frac{n_{i}}{n} U_{i}-\sum_{1 \leq i<i^{\prime} \leq k} \frac{n_{i} n_{i^{\prime}}}{n(n-1)} U_{i}-\sum_{1 \leq i<i^{\prime} \leq k} \frac{n_{i} n_{i^{\prime}}}{n(n-1)} U_{i^{\prime}},
\end{aligned}
$$

sendo esta última identidade obtida através de um argumento de simetria. Por conseguinte, podemos reescrever (3.9) como

$$
\begin{aligned}
U_{n}^{0} & =\sum_{i=1}^{k} \frac{n_{i}}{n} U_{i}-\sum_{1 \leq i<i^{\prime} \leq k} \frac{n_{i} n_{i^{\prime}}}{n(n-1)} U_{i}-\sum_{1 \leq i<i^{\prime} \leq k} \frac{n_{i} n_{i^{\prime}}}{n(n-1)} U_{i^{\prime}}+2 \sum_{1 \leq i<i^{\prime} \leq k} \frac{n_{i} n_{i^{\prime}}}{n(n-1)} U_{i i^{\prime}} \\
& =\sum_{i=1}^{k} \frac{n_{i}}{n} U_{i}+\sum_{1 \leq i<i^{\prime} \leq k} \frac{n_{i} n_{i^{\prime}}}{n(n-1)}\left\{2 U_{i i^{\prime}}-U_{i}-U_{i^{\prime}}\right\} \\
& =W_{n}+B_{n},
\end{aligned}
$$

em que $W_{n}=\sum_{i=1}^{k} \frac{n_{i}}{n} U_{i}$ e $B_{n}=\sum_{1 \leq i<i^{\prime} \leq k} \frac{n_{i} n_{i^{\prime}}}{n(n-1)}\left\{2 U_{i i^{\prime}}-U_{i}-U_{i^{\prime}}\right\}$. Esse tipo de decomposição foi utilizada em Sen $(1999,2006)$ e Pinheiro et al. $(2005,2007)$ com outros propósitos. 
Agora, notemos que

$$
\begin{aligned}
\mathbb{E}\left[B_{n}\right] & =\sum_{1 \leq i<i^{\prime} \leq k} \frac{n_{i} n_{i^{\prime}}}{n(n-1)}\left\{2\left(\sigma_{b}^{2}+\sigma_{e}^{2}\right)-2 \sigma_{e}^{2}\right\} \\
& =2 \sigma_{b}^{2} \sum_{1 \leq i<i^{\prime} \leq k} \frac{n_{i} n_{i^{\prime}}}{n(n-1)}=\sigma_{b}^{2} \sum_{i=1}^{k} \sum_{i^{\prime} \neq i} \frac{n_{i} n_{i^{\prime}}}{n(n-1)}=\sigma_{b}^{2} \sum_{i=1}^{k} \frac{n_{i}\left(n-n_{i}\right)}{n(n-1)} \\
& =\sigma_{b}^{2}\left(\frac{n^{2}-\sum_{i=1}^{k} n_{i}^{2}}{n(n-1)}\right),
\end{aligned}
$$

implicando que $\mathbb{E}\left[B_{n}\right]>0$ se e somente se $\sigma_{b}^{2}>0$ e $\mathbb{E}\left[B_{n}\right]=0$ se e somente se $\sigma_{b}^{2}=0$. Este fato sugere que a estatística $B_{n}$ (devidamente padronizada) pode ser utilizada para definir um teste para a hipótese de que $\sigma_{b}^{2}=0$, como veremos a seguir.

Primeiramente, usando os procedimentos descritos no capítulo anterior, concluímos que a decomposição $H$ de $U_{i}(i=1, \ldots, k)$ é dada por

$$
U_{i}=\sigma_{e}^{2}+\left\{\frac{1}{n_{i}} \sum_{j=1}^{n_{i}}\left(Y_{i j}-\mu\right)^{2}-\sigma_{e}^{2}\right\}-\left(\begin{array}{c}
n_{i} \\
2
\end{array}\right)^{-1} \sum_{1 \leq j<j^{\prime} \leq n_{i}}\left(Y_{i j}-\mu\right)\left(Y_{i j^{\prime}}-\mu\right) .
$$

Por outro lado, a decomposição $H$ da estatística generalizada $U_{i i^{\prime}}$ (para detalhes, veja por exemplo, Lee, 1990, p.40) é dada por

$$
U_{i i^{\prime}}=\left(\sigma_{e}^{2}+\sigma_{b}^{2}\right)+H_{i i^{\prime}}^{(1,0)}+H_{i i^{\prime}}^{(0,1)}+H_{i i^{\prime}}^{(1,1)},
$$

em que

$$
\begin{aligned}
& H_{i i^{\prime}}^{(1,0)}=n_{i}^{-1} \sum_{j=1}^{n_{i}} h^{(1,0)}\left(Y_{i j}\right), \\
& H_{i i^{\prime}}^{(0,1)}=n_{i^{\prime}}^{-1} \sum_{j^{\prime}=1}^{n_{i^{\prime}}} h^{(0,1)}\left(Y_{i^{\prime} j^{\prime}}\right), \\
& H_{i i^{\prime}}^{(1,1)}=\left(n_{i} n_{i^{\prime}}\right)^{-1} \sum_{j=1}^{n_{i}} \sum_{j^{\prime}=1}^{n_{i^{\prime}}} h^{(1,1)}\left(Y_{i j}, Y_{i^{\prime} j^{\prime}}\right)
\end{aligned}
$$

com

$$
\begin{aligned}
h^{(1,0)}\left(Y_{i j}\right) & =\mathbb{E}\left[g\left(Y_{i j}, Y_{i^{\prime} j^{\prime}}\right) \mid Y_{i j}\right]-\mathbb{E}\left[g\left(Y_{i j}, Y_{i^{\prime} j^{\prime}}\right)\right]=\left\{\left(Y_{i j}-\mu\right)^{2}-\left(\sigma_{e}^{2}+\sigma_{b}^{2}\right)\right\} / 2, \\
h^{(0,1)}\left(Y_{i^{\prime} j^{\prime}}\right) & =\left\{\left(Y_{i^{\prime} j^{\prime}}-\mu\right)^{2}-\left(\sigma_{e}^{2}+\sigma_{b}^{2}\right)\right\} / 2, \\
h^{(1,1)}\left(Y_{i j}, Y_{i^{\prime} j^{\prime}}\right) & =\mathbb{E}\left[g\left(Y_{i j}, Y_{i^{\prime} j^{\prime}}\right) \mid Y_{i j}, Y_{i^{\prime} j^{\prime}}\right]-h^{(1,0)}\left(Y_{i j}\right)-h^{(0,1)}\left(Y_{i^{\prime} j^{\prime}}\right)+\mathbb{E}\left[g\left(Y_{i j}, Y_{i^{\prime} j^{\prime}}\right)\right] \\
& =\left\{\left(Y_{i j}-Y_{i^{\prime} j^{\prime}}\right)^{2}-\left(Y_{i j}-\mu\right)^{2}-\left(Y_{i^{\prime} j^{\prime}}-\mu\right)^{2}\right\} / 2 \\
& =-\left(Y_{i j}-\mu\right)\left(Y_{i^{\prime} j^{\prime}}-\mu\right) .
\end{aligned}
$$

Nobre, Juvêncio S. 
Então, mais explicitamente, a decomposição (3.12) pode ser escrita como

$$
\begin{aligned}
U_{i i^{\prime}} & =\left(\sigma_{e}^{2}+\sigma_{b}^{2}\right)+\frac{1}{2}\left\{\frac{1}{n_{i}} \sum_{j=1}^{n_{i}}\left(Y_{i j}-\mu\right)^{2}-\left(\sigma_{e}^{2}+\sigma_{b}^{2}\right)\right\} \\
& +\frac{1}{2}\left\{\frac{1}{n_{i^{\prime}}} \sum_{j^{\prime}=1}^{n_{i^{\prime}}}\left(Y_{i^{\prime} j^{\prime}}-\mu\right)^{2}-\left(\sigma_{e}^{2}+\sigma_{b}^{2}\right)\right\} \\
& -\frac{1}{n_{i} n_{i^{\prime}}} \sum_{j=1}^{n_{i}} \sum_{j^{\prime}=1}^{n_{i^{\prime}}}\left(Y_{i j}-\mu\right)\left(Y_{i^{\prime} j^{\prime}}-\mu\right) .
\end{aligned}
$$

Lembrando que $\mathbf{Y}_{i}=\left(Y_{i 1}, \ldots, Y_{i n_{i}}\right)^{\top}, i=1, \ldots, k$, então por (3.11) e (3.13) temos

$$
\begin{aligned}
\phi^{*}\left(\mathbf{Y}_{i}, \mathbf{Y}_{i^{\prime}}\right) & =2 U_{i i^{\prime}}-U_{i}-U_{i^{\prime}}=2\left(\sigma_{e}^{2}+\sigma_{b}^{2}\right)+\left\{\frac{1}{n_{i}} \sum_{j=1}^{n_{i}}\left(Y_{i j}-\mu\right)^{2}-\left(\sigma_{e}^{2}+\sigma_{b}^{2}\right)\right\} \\
& +\left\{\frac{1}{n_{i^{\prime}}} \sum_{j^{\prime}=1}^{n_{i^{\prime}}}\left(Y_{i^{\prime} j^{\prime}}-\mu\right)^{2}-\left(\sigma_{e}^{2}+\sigma_{b}^{2}\right)\right\}-\frac{2}{n_{i} n_{i^{\prime}}} \sum_{j=1}^{n_{i}} \sum_{j^{\prime}=1}^{n_{i^{\prime}}}\left(Y_{i j}-\mu\right)\left(Y_{i^{\prime} j^{\prime}}-\mu\right) \\
& -\sigma_{e}^{2}-\left\{\frac{1}{n_{i}} \sum_{j=1}^{n_{i}}\left(Y_{i j}-\mu\right)^{2}-\sigma_{e}^{2}\right\}+\left(\begin{array}{c}
n_{i} \\
2
\end{array}\right)^{-1} \sum_{1 \leq j<j^{\prime} \leq n_{i}}\left(Y_{i j}-\mu\right)\left(Y_{i j^{\prime}}-\mu\right) \\
& -\sigma_{e}^{2}-\left\{\frac{1}{n_{i^{\prime}}} \sum_{j^{\prime}=1}^{n_{i^{\prime}}}\left(Y_{i^{\prime} j^{\prime}}-\mu\right)^{2}-\sigma_{e}^{2}\right\}+\left(\begin{array}{c}
n_{i^{\prime}} \\
2
\end{array}\right)^{-1} \sum_{1 \leq k<k^{\prime} \leq n_{i^{\prime}}}\left(Y_{i^{\prime} k}-\mu\right)\left(Y_{i^{\prime} k^{\prime}}-\mu\right) \\
& =\phi_{0}+\psi_{2}\left(\mathbf{Y}_{i}, \mathbf{Y}_{i^{\prime}}\right),
\end{aligned}
$$

em que $\phi_{0}=\mathbb{E}\left[\phi^{*}\left(\mathbf{Y}_{i}, \mathbf{Y}_{i^{\prime}}\right)\right]=2 \sigma_{b}^{2}$, e

$$
\begin{aligned}
\psi_{2}\left(\mathbf{Y}_{i}, \mathbf{Y}_{i^{\prime}}\right) & =\left(\begin{array}{c}
n_{i} \\
2
\end{array}\right)^{-1} \sum_{1 \leq j<j^{\prime} \leq n_{i}}\left(Y_{i j}-\mu\right)\left(Y_{i j^{\prime}}-\mu\right) \\
& +\left(\begin{array}{c}
n_{i^{\prime}} \\
2
\end{array}\right)^{-1} \sum_{1 \leq k<k^{\prime} \leq n_{i^{\prime}}}\left(Y_{i^{\prime} k}-\mu\right)\left(Y_{i^{\prime} k^{\prime}}-\mu\right) \\
& -\frac{2}{n_{i} n_{i^{\prime}}} \sum_{j=1}^{n_{i}} \sum_{j^{\prime}=1}^{n_{i^{\prime}}}\left(Y_{i j}-\mu\right)\left(Y_{i^{\prime} j^{\prime}}-\mu\right)-2 \sigma_{b}^{2},
\end{aligned}
$$

o que implica que $2 U_{i i^{\prime}}-U_{i}-U_{i^{\prime}}$ é uma estatística degenerada de primeira ordem (no sentido utilizado em Pinheiro et al., 2007) ${ }^{1}$. Sob a hipótese $\mathcal{H}_{0}: \sigma_{b}^{2}=0$, temos $\phi_{0}=0$ e

$$
\begin{aligned}
\psi_{2}\left(\mathbf{Y}_{i}, \mathbf{Y}_{i^{\prime}}\right) & =\left(\begin{array}{c}
n_{i} \\
2
\end{array}\right)^{-1} \sum_{1 \leq j<j^{\prime} \leq n_{i}} \psi\left(Y_{i j}, Y_{i j^{\prime}}\right)+\left(\begin{array}{c}
n_{i^{\prime}} \\
2
\end{array}\right)^{-1} \sum_{1 \leq k<k^{\prime} \leq n_{i^{\prime}}} \psi\left(Y_{i^{\prime} k}, Y_{i^{\prime} k^{\prime}}\right) \\
& -\frac{2}{n_{i} n_{i^{\prime}}} \sum_{j=1}^{n_{i}} \sum_{j^{\prime}=1}^{n_{i^{\prime}}} \psi\left(Y_{i j}, Y_{i^{\prime} j^{\prime}}\right),
\end{aligned}
$$

${ }^{1}$ Ou seja as funções de grau 1 de $\mathbf{Y}_{i}$ e $\mathbf{Y}_{i^{\prime}}$ separadamente são variáveis aleatórias nulas com probabilidade 1.

Nobre, Juvêncio S.

IME-USP 
com $\psi\left(x_{1}, x_{2}\right)=\left(x_{1}-\mu\right)\left(x_{2}-\mu\right)$. Além disso, é possível mostrar que a estatística $B_{n}$ definida em (3.10) pode ser reescrita como (veja Apêndice B)

$$
B_{n}=\left(\begin{array}{l}
n \\
2
\end{array}\right)^{-1} \sum_{1 \leq r<s \leq n} \eta_{n r s} \psi\left(Y_{r}, Y_{s}\right)
$$

em que

$$
\eta_{n r s}= \begin{cases}\frac{n-n_{i}}{n_{i}-1} & , \text { se } Y_{r} \text { e } Y_{s} \text { são observações da } i \text {-ésima unidade amostral, } \\ -1 & , \text { em caso contrário. }\end{cases}
$$

Além do mais, é possível mostrar que os coeficientes $\eta$ 's são tais que (veja Apêndice B)

$$
\sum_{1 \leq r<s \leq n} \eta_{n r s}=0
$$

$\mathrm{e}$

$$
\sum_{1 \leq r<s \leq n} \eta_{n r s}^{2}=\left(\begin{array}{l}
n \\
2
\end{array}\right)(k-1)\left\{1+\frac{1}{n} \sum_{i=1}^{k} \frac{n-n_{i}}{\left(n_{i}-1\right)(k-1)}\right\} .
$$

Considerando $k \rightarrow \infty$ e assumindo que os $n_{i}$ 's são limitados, temos $k=O(n)$ o que implica por (3.18), que $M_{n}=\sum_{1 \leq r<s \leq n} \eta_{n r s}^{2}=O\left(n^{3}\right)$.

Sob $\mathcal{H}_{0}: \sigma_{b}^{2}=0$, as variáveis aleatórias $Y_{1}, \ldots, Y_{n}$ são iid e a função núcleo $\psi(x, y)$ é estacionária de primeira ordem formando um sistema ortogonal (no sentido adotado em Pinheiro et al., 2007), isto é,

$$
\begin{aligned}
\psi_{1}\left(Y_{s}\right) & =\mathbb{E}\left[\psi\left(Y_{r}, Y_{s}\right) \mid Y_{s}\right] \\
& =\mathbb{E}\left[\left(Y_{r}-\mu\right)\left(Y_{s}-\mu\right) \mid Y_{s}\right]=0 \text { quase certamente (q.c.) } \\
\mathbb{E}\left[\psi\left(Y_{1}, Y_{2}\right) \psi\left(Y_{1}, Y_{3}\right)\right] & =0
\end{aligned}
$$

Além disso, pela suposição de independência, temos que $\mathbb{E}\left[\psi^{2}\left(Y_{r}, Y_{s}\right)\right]=\operatorname{Var}\left[\psi\left(Y_{r}, Y_{s}\right)\right]=$ $\mathbb{E}\left[\left(Y_{r}-\mu\right)^{2}\left(Y_{s}-\mu\right)^{2}\right]=\operatorname{Var}\left[Y_{r}\right] \operatorname{Var}\left[Y_{s}\right]=\sigma_{e}^{4}<\infty$. Adicionalmente, consideremos a seguinte estatística

$$
J_{n}=\frac{\left(\begin{array}{c}
n \\
2
\end{array}\right) B_{n}}{W_{n} \sqrt{M_{n}}}
$$

Nosso resultado fundamental é baseado no seguinte teorema:

Teorema 3.1. Consideremos o modelo (3.1) e a estatística $J_{n}$ definida em (3.21). Sob as suposições (3.2)-(3.4) e admitamos adicionalmente que $\left\{n_{i}\right\}_{i=1, \ldots, k}$ é limitada. Então, sob $\mathcal{H}_{0}: \sigma_{b}^{2}=0$,

$$
J_{n} \stackrel{\mathcal{D}}{\longrightarrow} \mathcal{N}(0,1),
$$

quando $k \rightarrow \infty(\Rightarrow n \rightarrow \infty)$. 
Detalhes da demonstração são apresentados no Apêndice B. Podemos utilizar $J_{n}$ como estatística de teste para $\mathcal{H}_{0}: \sigma_{b}^{2}=0$ versus $\mathcal{H}_{1}: \sigma_{b}^{2}>0$. Como, sob $\mathcal{H}_{1}$, a esperança assintótica da estatística $J_{n}$ é positiva, a hipótese nula $\mathcal{H}_{0}$ é rejeitada, com nível de significância $\alpha \in(0,1)$ se $J_{n} \geq z_{\alpha}$, com $z_{\alpha}$ representando o quantil de ordem $(1-\alpha)$ da distribuição normal padrão.

Como $\lim _{n \rightarrow \infty} P\left[J_{n} \geq z_{\alpha} \mid \sigma_{b}^{2}>0\right]=1$, ou seja, como o poder do teste para hipóteses fixas sempre converge para 1 , vamos restringir o estudo a hipóteses locais de Pitman do tipo

$$
\mathcal{H}_{1 n}: \sigma_{b}^{2}=\delta^{2} / \sqrt{n}
$$

com $\delta$ representando uma constante. Lembrando que $M_{n}=O\left(n^{3}\right)$ e admitindo a existência do quarto momento do efeito aleatório, podemos encontrar a distribuição assintótica da estatística $J_{n}$ para a seqüência de hipóteses locais de Pitman (3.22), por intermédio do seguinte teorema:

Teorema 3.2. Consideremos o modelo (3.1) sob a seqüência $\mathcal{H}_{1 n}: \sigma_{b}^{2}=\delta^{2} / \sqrt{n}$ e a estatística $J_{n}$ definida em (3.21). Denotando $\lim _{n \rightarrow \infty} M_{n} / n^{3}=\lambda$, admitindo que $\mathbb{E}\left[b_{i}^{4}\right]<\infty$ e considerando as hipóteses do Teorema 3.1, temos que

$$
J_{n} \stackrel{\mathcal{D}}{\longrightarrow} \mathcal{N}\left(\frac{\delta^{2}}{2 \sigma_{e}^{2} \sqrt{\lambda}}, 1\right)
$$

quando $k \rightarrow \infty(\Rightarrow n \rightarrow \infty)$

O resultado acima indica que o poder do teste é uma função monótona estritamente crescente do coeficiente de correlação intra-classe $\rho=\delta^{2} /\left(\delta^{2}+\sigma_{e}^{2}\right)$, como era de se esperar. É importante salientar que como conseqüência da contigüidade da seqüência (3.22) e de $\mathbb{E}\left[b_{i}^{4}\right]<\infty$, obtemos que $\mathbb{E}\left[b_{i}^{4}\right]=o(1)$ sob $\mathcal{H}_{1 n}$.

\subsubsection{Estudos de Simulação}

Para avaliar o comportamento do teste proposto em casos com amostras pequenas e de tamanho moderado, simulamos 10.000 réplicas de Monte-Carlo sob o modelo (3.1), com $\mu=2$ e $\sigma_{e}^{2}=1$ para diferentes números de unidades amostrais $(k=10,30,100)$ em estudos balanceados $\left(n_{i}=m, i=1, \ldots, k\right)$ considerando diferentes números de observações por unidade amostral $(m=2,4,5,10)$.

Assumimos inicialmente que $e_{i j} \sim \mathcal{N}(0,1)$ e $b_{i} \sim t_{3} \times \sqrt{\sigma_{b}^{2} / 3}$, em que $t_{v}$ representa uma distribuição $t$ de Student com $v$ graus de liberdade, implicando que $\operatorname{Var}\left[b_{i}\right]=\sigma_{b}^{2}$. Para $\sigma_{b}^{2}$, consideramos os valores $0,0.2,0.5$ e 1 . O poder do teste para cada configuração foi estimado 
empiricamente (i.e., como a proporção de vezes em que se rejeitou a hipótese nula), para o nível de significância em questão (1\%, 5\% e 10\%). Para ilustrar a robustez do teste, quando se considera uma distribuição com caudas mais pesadas que a da normal para os erros condicionais, repetimos o estudo de simulação supracitado com $e_{i j} \sim t_{5} \times \sqrt{3 / 5}$, implicando que $\operatorname{Var}\left[e_{i j}\right]=1$. Os resultados estão dispostos na Tabela 3.1.

Tabela 3.1 Estimativas do poder do teste (\%) de $\mathcal{H}_{0}: \sigma_{b}^{2}=0$ sob o modelo (3.1).

\begin{tabular}{|c|c|c|c|c|c|c|c|c|c|c|c|c|c|}
\hline \multirow[b]{2}{*}{$\sigma_{b}^{2}$} & \multirow[b]{2}{*}{$\alpha$} & \multicolumn{4}{|c|}{$k=10$} & \multicolumn{4}{|c|}{$k=30$} & \multicolumn{4}{|c|}{$k=100$} \\
\hline & & $m=2$ & $m=4$ & $m=5$ & $m=10$ & $m=2$ & $m=4$ & $m=5$ & $m=10$ & $m=2$ & $m=4$ & $m=5$ & $m=10$ \\
\hline \multicolumn{14}{|c|}{$e_{i j} \sim \mathcal{N}(0,1)$} \\
\hline \multirow{3}{*}{0} & $1 \%$ & 8,5 & 3,7 & 3,0 & 1,6 & 4,2 & 2,8 & 2,3 & 1,7 & 2,9 & 1,8 & 1,5 & 1,2 \\
\hline & $5 \%$ & 14,0 & 8,8 & 7,6 & 6,2 & 8,3 & 7,1 & 6,3 & 6,1 & 7,2 & 6,1 & 5,9 & 5,4 \\
\hline & $10 \%$ & 18,7 & 13,5 & 12,7 & 11,3 & 13,6 & 12,1 & 11,7 & 11,0 & 12,7 & 11,0 & 10,9 & 10,4 \\
\hline 0.2 & $10 \%$ & 32,1 & 43,1 & 49,0 & 70,2 & 40,8 & 66,8 & 75,8 & 95,3 & 63,6 & 94,9 & 98,4 & 100,0 \\
\hline \multirow{3}{*}{0.5} & $1 \%$ & 30,9 & 50,7 & 58,5 & 83,5 & 48,0 & 84,1 & 91,3 & 99,6 & 83,8 & 99,8 & 100,0 & 100,0 \\
\hline & $5 \%$ & 40,6 & 61,0 & 68,8 & 89,2 & 61,3 & 91,4 & 95,6 & 99,8 & 92,6 & 99,9 & 100,0 & 100,0 \\
\hline & $10 \%$ & 47,2 & 68,3 & 75,0 & 90,8 & 69,4 & 93,9 & 97,0 & 99,9 & 94,9 & 99,9 & 100,0 & 100,0 \\
\hline \multicolumn{14}{|c|}{$e_{i j} \sim t_{5} \times(3 / 5)^{1 / 2}$} \\
\hline \multirow{3}{*}{0} & $1 \%$ & 8,0 & 3,6 & 3,2 & 2,1 & 4,1 & 2,1 & 2,1 & 1,9 & 2,9 & 1,7 & 1,5 & 1,2 \\
\hline & $5 \%$ & 12,7 & 8,9 & 7,8 & 6,3 & 8,4 & 6,8 & 6,3 & 6,1 & 7,3 & 5,9 & 5,5 & 5,3 \\
\hline & $10 \%$ & 17,2 & 13,0 & 12,6 & 11,0 & 13,8 & 11,4 & 11,0 & 10,7 & 12,7 & 11,1 & 11,0 & 10,3 \\
\hline \multirow{3}{*}{0.2} & $1 \%$ & 18,5 & 27,9 & 32,0 & 53,1 & 21,0 & 45,8 & 56,2 & 87,6 & 37,5 & 82,9 & 93,1 & 99,9 \\
\hline & $5 \%$ & 26,4 & 36,9 & 42,2 & 64,0 & 33,7 & 61,1 & 68,8 & 92,9 & 54,1 & 91,8 & 97,5 & 100,0 \\
\hline & $10 \%$ & 32,3 & 44,2 & 49,5 & 69,9 & 41,4 & 68,2 & 77,1 & 95,4 & 64,1 & 94,9 & 98,3 & 100,0 \\
\hline \multirow{3}{*}{0.5} & $1 \%$ & 32,6 & 52,1 & 61,7 & 83,0 & 48,8 & 84,7 & 91,3 & 99,5 & 83,8 & 99,8 & 100,0 & 100,0 \\
\hline & $5 \%$ & 41,2 & 62,5 & 70,9 & 88,7 & 62,5 & 90,9 & 95,4 & 99,9 & 91,8 & 100,0 & 100,0 & 100,0 \\
\hline & $10 \%$ & 48,2 & 69,4 & 75,8 & 91,5 & 69,7 & 92,9 & 97,1 & 99,9 & 95,2 & 100,0 & 100,0 & 100,0 \\
\hline
\end{tabular}

Os resultados da parte superior da Tabela 3.1, obtidos sob normalidade do erro condicional, indicam que o teste tende a ser liberal (seu tamanho é maior que o nível de significância), principalmente quando se tem poucas observações para cada unidade amostral $(m \leq 4)$. Este viés diminui rapidamente à medida que aumenta o número de unidades amostrais e/ou o número de observações por unidade amostral. Quando se tem ao menos 5 observações por unidade amostral e pelo menos 30 unidades amostrais, a diferença (absoluta) entre o tamanho do teste e o nível de significância é menor que $2 \%$. Analisando a tabela, temos uma idéia a respeito do aumento do poder em função de $\sigma_{b}^{2}, m$ e $k$. Quando simulamos a distribuição do erro condicional através de uma distribuição $t$ (parte inferior da Tabela 3.1), podemos 
notar um comportamento similar ao da situação anterior; nesse caso, o tamanho do teste fica mais próximo do nível de significância na maioria das situações.

\subsubsection{Comparação entre os testes $F$ e $J_{n}$}

O objetivo desta subseção é comparar o desempenho do teste proposto com o do teste exato $F$ (veja por exemplo, Khuri et al.,1998 e Demidenko,2004), para testar $\sigma_{b}^{2}=0$ sob o modelo (3.1). O teste $F$ é obtido sob as suposições de independência e normalidade por parte dos efeitos aleatórios e do erro condicional. A estatítica do teste é

$$
F_{1}=\frac{\mathrm{SQ}(b) /(k-1)}{\mathrm{SQ}(e) /(n-k)}
$$

em que $\mathrm{SQ}(b)=\sum_{i=1}^{k} n_{i}\left(\bar{Y}_{i .}-\bar{Y}_{. .}\right)^{2}$ e $\mathrm{SQ}(e)=\sum_{i=1}^{k} \sum_{j=1}^{n_{i}} Y_{i j}^{2}-\sum_{i=1}^{k} n_{i} \bar{Y}_{i .}^{2}$, correspondendo respectivamente à soma de quadrados devida aos efeitos aleatórios e à soma de quadrados do resíduo. Esse teste é obtido usando o mesmo princípio dos testes usuais de ANOVA sob o modelo (3.1). Khuri et al. (1998) mostram que, sob $\mathcal{H}_{0}: \sigma_{b}^{2}=0$, a estatística $F_{1}$ possui uma distribuição exata $F(k-1, n-k)$. Portanto, com base em alguma amostra gerada sob o modelo (3.1), rejeitamos a hipótese de inexistência do efeito aleatório, se $F_{1}>F_{1-\alpha}(k-1, n-k)$, com $F_{1-\alpha}(k-1, n-k)$ representando o quantil de ordem (1 $\alpha)$ da distribuição $F(k-1, n-k)$. Se o estudo for balanceado, o referido teste é o teste invariante uniformemente mais poderoso (IUMP) (uniformly most powerful invariant test) ${ }^{2}$. Se o estudo não for balanceado, não existe teste IUMP. Khuri et al. (1998) comentam sobre o efeito do desbalanceamento na diminuição do poder do teste em questão. Para uma análise deste efeito, veja por exemplo, Donner e Koval (1989) e Lee (2003). Demidenko (2004, p. 137) propõe um teste exato para verificar a significância conjunta de todos os candidatos a efeitos aleatórios sob o modelo geral (1.2). Tal teste será discutido com mais detalhes na Seção 3.3. Para o caso específico em questão, a estatística do teste proposto se reduz à estatística $F_{1}$ supracitada.

Para comparar os dois testes, repetimos o processo de simulação descrito na seção anterior, utilizando os mesmos valores para $\mu$ e $\sigma_{e}^{2}$, em um estudo balanceado, com 5 observações por unidade amostral. Em um primeiro instante, os efeitos aleatórios e o vetor de erros condicionais foram, respectivamente gerados da seguinte forma: $b_{i} \sim \mathcal{N}\left(0, \sigma_{b}^{2}\right)$ e $e_{i j} \sim \mathcal{N}(0,1)$. Os resultados, apresentados na Tabela 3.2, indicam que o teste baseado na estatística $J_{n}$ tem uma característica liberal, mais evidente quando se tem poucas unidades amostrais,

${ }^{2}$ Com relação ao grupo de transformações $\mathbf{Y} \rightarrow c\left(\mathbf{Y}+a \mathbf{1}_{n}\right)$, com $c>0, a \in \mathbb{R}$ e $\mathbf{1}_{n}$ denotando um vetor de dimensão $n \times 1$ com todos elementos iguais a 1. Para maiores detalhes, veja Khuri et al. (1998, p.97).

Nobre, Juvêncio S. 
sendo a diferença (absoluta) entre o tamanho do teste e o nível de significância na ordem de $2 \%$. Quando aumentamos o número de unidades amostrais e/ou observações por unidade amostral, tanto o tamanho quanto o poder do teste ficam próximos daqueles do teste $F$.

Tabela 3.2 Estimativas do poder do teste (\%) de $\mathcal{H}_{0}: \sigma_{b}^{2}=0$ sob o modelo (3.1) para um estudo balanceado, com 5 observações por unidade amostral.

\begin{tabular}{|c|c|c|c|c|c|c|c|c|c|c|c|}
\hline \multirow[b]{2}{*}{$\sigma_{b}^{2}$} & \multirow[b]{2}{*}{$\alpha$} & \multicolumn{2}{|c|}{$k=10$} & \multicolumn{2}{|c|}{$k=20$} & \multicolumn{2}{|c|}{$k=30$} & \multicolumn{2}{|c|}{$k=50$} & \multicolumn{2}{|c|}{$k=100$} \\
\hline & & $F$ & $J_{n}$ & $F$ & $J_{n}$ & $F$ & $J_{n}$ & $F$ & $J_{n}$ & $F$ & $J_{n}$ \\
\hline \multirow{3}{*}{0} & $1 \%$ & 0,9 & 3,1 & 1,0 & 2,8 & 0,8 & 2,2 & 1,1 & 2,0 & 1,0 & 1,5 \\
\hline & $5 \%$ & 4,9 & 7,6 & 4,7 & 7,2 & 5,3 & 7,0 & 4,8 & 5,9 & 4,9 & 5,6 \\
\hline & $10 \%$ & 9,7 & 12,3 & 9,9 & 11,9 & 10,5 & 11,4 & 10,2 & 10,9 & 9,6 & 10,3 \\
\hline \multirow{3}{*}{0.2} & $1 \%$ & 20,1 & 38,2 & 39,2 & 55,9 & 57,0 & 69,8 & 80,0 & 86,9 & 98,1 & 99,0 \\
\hline & $5 \%$ & 41,1 & 50,9 & 63,0 & 70,8 & 77,6 & 82,4 & 92,3 & 94,0 & 99,5 & 99,7 \\
\hline & $10 \%$ & 54,5 & 59,2 & 74,2 & 77,1 & 85,8 & 88,1 & 96,1 & 96,4 & 99,9 & 99,9 \\
\hline \multirow{3}{*}{0.5} & $1 \%$ & 58,9 & 77,1 & 89,0 & 94,2 & 97,4 & 98,9 & 99,9 & 100,0 & 100,0 & 100,0 \\
\hline & $5 \%$ & 78,3 & 84,3 & 95,5 & 97,3 & 99,3 & 99,5 & 100,0 & 100,0 & 100,0 & 100,0 \\
\hline & $10 \%$ & 85,6 & 88,2 & 98,0 & 98,3 & 99,7 & 99,8 & 100,0 & 100,0 & 100,0 & 100,0 \\
\hline \multirow{3}{*}{1} & $1 \%$ & 87,5 & 94,3 & 99,3 & 99,8 & 99,8 & 100,0 & 100,0 & 100,0 & 100,0 & 100,0 \\
\hline & $5 \%$ & 95,1 & 96,7 & 99,8 & 99,9 & 100,0 & 100,0 & 100,0 & 100,0 & 100,0 & 100,0 \\
\hline & $10 \%$ & 97,2 & 97,8 & 99,9 & 99,9 & 100,0 & 100,0 & 100,0 & 100,0 & 100,0 & 100,0 \\
\hline
\end{tabular}

Para ilustrar o efeito que o desbalanceamento pode causar nos resultados, repetimos o processo de simulação, sendo que para cada unidade amostral selecionamos equiprovavelmente o número de observações no conjunto $\{5,6,7,8,9,10\}$. Além disso, para avaliar o possível efeito da não-normalidade dos efeitos aleatórios e do erro condicional no comportamento do teste exato $F$, simulamos as distribuições de $b_{i}$ e $e_{i j}$ com caudas mais pesadas que a da distribuição normal padrão, a saber, $b_{i} \sim t_{4,1} \times \sqrt{\sigma_{b}^{2}(2,1 / 4,1)}$ e $e_{i j} \sim t_{4,1} \times \sqrt{(2,1 / 4,1)}$, de tal forma que $\operatorname{Var}\left[b_{i}\right]=\sigma_{b}^{2}$ e $\operatorname{Var}\left[e_{i j}\right]=1$.

Para medir o grau de desbalanceamento seguimos as sugestões de Donner e Koval (1989) e Khuri et al. (1998) e usamos o parâmetro

$$
\kappa=\frac{1}{1+\vartheta^{2}}
$$

em que $\vartheta$ representa o coeficiente de variação associado à amostra $n_{1}, \ldots, n_{k}, \operatorname{com} n_{i}(i=$ $1, \ldots, k)$ representando o número de observações da $i$-ésima unidade amostral. Pela definição, $0<\kappa \leq 1$, sendo $\kappa=1$ somente em estudos balanceados. Quanto menor é o valor de $\kappa$, maior é grau de desbalanceamento. Para essa situação, geramos os números de observações 
para cada cada unidade amostral de forma que o grau de desbalanceamento associado à distribuição de probabilidades de $n_{1}, \ldots, n_{k}$ fosse pequeno, especificamente $\kappa \cong 0,95$.

Tabela 3.3 Estimativas do poder do teste (\%) de $\mathcal{H}_{0}: \sigma_{b}^{2}=0$ sob o modelo (3.1) para um estudo desbalanceado, com o número de observações por unidade amostral sendo selecionado de forma equiprovável no conjunto $\{5,6,7,8,9,10\}$.

\begin{tabular}{rccrrrrrrrrrr}
\hline \hline & \multicolumn{1}{c}{$k=10$} & \multicolumn{3}{c}{$k=20$} & \multicolumn{3}{c}{$k=30$} & \multicolumn{3}{c}{$k=50$} & \multicolumn{2}{c}{$k=100$} \\
$\sigma_{b}^{2}$ & $\alpha$ & $F$ & $J_{n}$ & $F$ & $J_{n}$ & $F$ & $J_{n}$ & $F$ & $J_{n}$ & $F$ & $J_{n}$ \\
\hline \hline & $1 \%$ & 0,6 & 2,1 & 0,7 & 1,8 & 0,8 & 1,6 & 1,2 & 1,3 & 1,7 & 1,2 \\
0 & $5 \%$ & 3,5 & 5,9 & 3,9 & 5,5 & 4,4 & 5,4 & 4,7 & 5,3 & 5,3 & 5,1 \\
& $10 \%$ & 8,1 & 11,4 & 8,1 & 10,4 & 9,0 & 10,3 & 9,4 & 10,2 & 10,6 & 9,8 \\
\hline \hline & $1 \%$ & 32,3 & 46,0 & 46,5 & 62,0 & 59,2 & 71,2 & 73,4 & 82,7 & 87,2 & 93,5 \\
0.2 & $5 \%$ & 46,7 & 54,7 & 61,4 & 71,6 & 70,6 & 79,8 & 82,4 & 89,0 & 92,5 & 95,4 \\
& $10 \%$ & 55,1 & 62,1 & 70,4 & 76,9 & 77,6 & 84,4 & 88,0 & 92,0 & 94,6 & 96,9 \\
\hline \hline & $1 \%$ & 57,2 & 71,2 & 77,4 & 86,1 & 84,9 & 91,6 & 92,3 & 96,0 & 96,8 & 98,3 \\
0.5 & $5 \%$ & 68,0 & 79,3 & 85,4 & 90,3 & 91,2 & 94,4 & 95,7 & 97,7 & 98,0 & 98,8 \\
& $10 \%$ & 76,9 & 82,8 & 89,1 & 92,3 & 93,0 & 95,7 & 96,8 & 97,8 & 98,3 & 99,2 \\
\hline \hline & $1 \%$ & 78,8 & 85,2 & 90,8 & 95,0 & 94,1 & 97,5 & 97,3 & 98,6 & 98,7 & 99,3 \\
1 & $5 \%$ & 83,8 & 90,1 & 93,9 & 96,8 & 96,9 & 98,0 & 98,4 & 99,1 & 99,1 & 99,5 \\
& $10 \%$ & 88,0 & 91,7 & 96,1 & 97,2 & 97,4 & 98,7 & 98,6 & 99,3 & 99,3 & 99,6 \\
\hline \hline
\end{tabular}

Pela Tabela 3.3, percebemos que o tamanho do teste baseado em $J_{n}$ está bem próximo do nível de significância, mesmo quando se tem poucas unidades amostrais. O teste $F$ se mostra mais viesado. Em todas as configurações, o teste baseado na estatística $J_{n}$ se mostra mais poderoso, sendo maior o seu poder, comparativamente ao do teste $F$, em pequenas amostras. Para essa situação, percebemos um maior efeito do desbalanceamento bem como da não-normalidade, principalmente no poder do teste $F$.

Para ilustrar apenas o efeito do desbalanceamento no teste $F$, repetimos o processo de simulação descrito anteriormente, sendo que agora, para cada unidade amostral selecionamos o número de observações segundo uma distribuição geométrica com deslocamento de 2 unidades, ou seja, a distribuição de uma variável $Y=2+X$, com $X \sim \mathrm{G}(0,15)$ representando uma distribuição geométrica (com suporte em $\{0,1 \ldots\}$ ) com parâmetro 0,15 , implicando que o grau de desbalanceamento associado à distribuição de probabilidades geradora de $n_{1}, \ldots, n_{k}$ é $\kappa \cong 0,61$ e evidenciando um grau de desbalanceamento moderado. Os efeitos aleatórios e o vetor de erros condicionais foram gerados da seguinte forma: $b_{i} \sim \mathcal{N}\left(0, \sigma_{b}^{2}\right)$ e $e_{i j} \sim \mathcal{N}(0,1)$.

Na Tabela 3.4, observamos que apesar do moderado grau de desbalanceamento, o tamanho 
Tabela 3.4 Estimativas do poder do teste (\%) de $\mathcal{H}_{0}: \sigma_{b}^{2}=0$ sob o modelo (3.1) para um estudo desbalanceado, com o número de observações por unidade amostral sendo selecionado segundo uma distribuição $2+G(0,15)$.

\begin{tabular}{rrrrrrrrrrrrr}
\hline \hline & \multicolumn{1}{c}{$k=10$} & \multicolumn{2}{c}{$k=20$} & \multicolumn{2}{c}{$k=30$} & \multicolumn{2}{c}{$k=50$} & \multicolumn{2}{c}{$k=100$} \\
$\sigma_{b}^{2}$ & $\alpha$ & $F$ & $J_{n}$ & $F$ & $J_{n}$ & $F$ & $J_{n}$ & $F$ & $J_{n}$ & $F$ & $J_{n}$ \\
\hline \hline & $1 \%$ & 0,9 & 2,7 & 1,2 & 2,4 & 1,1 & 2,0 & 1,0 & 1,5 & 0,9 & 1,4 \\
0 & $5 \%$ & 5,2 & 7,2 & 5,0 & 6,7 & 5,1 & 6,2 & 4,9 & 6,0 & 4,9 & 5,7 \\
& $10 \%$ & 10,0 & 11,8 & 9,5 & 11,1 & 10,1 & 11,0 & 9,8 & 10,8 & 9,9 & 10,3 \\
\hline \hline & $1 \%$ & 42,1 & 53,1 & 65,8 & 81,6 & 83,5 & 95,0 & 96,6 & 98,2 & 99,4 & 100,0 \\
0.2 & $5 \%$ & 50,9 & 70,3 & 73,9 & 87,4 & 89,5 & 96,0 & 98,3 & 99,7 & 100,0 & 100,0 \\
& $10 \%$ & 56,5 & 77,5 & 86,9 & 91,7 & 95,3 & 98,5 & 98,9 & 99,7 & 100,0 & 100,0 \\
\hline \hline & \multirow{2}{*}{$1 \%$} & 85,3 & 97,4 & 99,8 & 99,7 & 100,0 & 100,0 & 100,0 & 100,0 & 100,0 & 100,0 \\
0.5 & $5 \%$ & 96,0 & 99,0 & 99,8 & 99,9 & 100,0 & 100,0 & 100,0 & 100,0 & 100,0 & 100,0 \\
& $10 \%$ & 97,8 & 99,6 & 100,0 & 100,0 & 100,0 & 100,0 & 100,0 & 100,0 & 100,0 & 100,0 \\
\hline \hline & \multirow{2}{*}{$1 \%$} & 98,4 & 99,4 & 100,0 & 100,0 & 100,0 & 100,0 & 100,0 & 100,0 & 100,0 & 100,0 \\
1 & $5 \%$ & 99,5 & 99,7 & 100,0 & 100,0 & 100,0 & 100,0 & 100,0 & 100,0 & 100,0 & 100,0 \\
& $10 \%$ & 99,6 & 99,9 & 100,0 & 100,0 & 100,0 & 100,0 & 100,0 & 100,0 & 100,0 & 100,0 \\
\hline \hline
\end{tabular}

do teste $F$ está bem próximo do nível nominal. O teste baseado na estatística $J_{n}$ mostra-se menos liberal do que nas duas situações apresentadas anteriormente, sendo seu viés menor à medida em que o número de unidades amostrais aumenta. Além disso, ele se mostra mais poderoso do que o teste $F$ na maioria das configurações.

Por fim, para ilustrar o efeito da não-normalidade bem como de assimetria da distribuição do erro condicional e do efeito aleatório no comportamento dos testes em um estudo balanceado com 5 observações por unidade amostral, simulamos $e_{i j}$ e $b_{i}$ utilizando distribuições assimétricas com caudas mais pesadas que a distribuição normal padrão, a saber, $b_{i} \sim$ $\left(\left(Y_{1}-\mathbb{E}\left[Y_{1}\right]\right) / \sqrt{\operatorname{Var}\left[Y_{1}\right]}\right) \times \sigma_{b}$, implicando que $\operatorname{Var}\left[b_{i}\right]=\sigma_{b}^{2}$, e $e_{i j} \sim\left(Y_{2}-\mathbb{E}\left[Y_{2}\right]\right) / \sqrt{\operatorname{Var}\left[Y_{2}\right]}$, com $Y_{1}, Y_{2}$ representando variáveis aleatórias iid com distribuição $t$ de Student assimétrica com parâmetro de localização 0, parâmetro de dispersão 1, com 4,1 graus de liberdade e parâmetro de assimetria $\lambda=1$, correspondendo a um coeficiente de assimetria aproximadamente igual a 1,77 (para detalhes sobre a distribuição $t$ assimétrica, veja Azzalini e Capitanio (2003) e Godoi (2007), por exemplo). Os resultados estão apresentados na Tabela 3.5.

O teste $F$ apresenta um comportamento conservador, principalmente quando o nível de significância é $10 \%$. Por outro lado, o tamanho do teste baseado na estatística $J_{n}$ está bem próximo do nível nominal, mesmo para amostras de tamanho moderado. Ele também se 
Tabela 3.5 Estimativas do poder do teste (\%) de $\mathcal{H}_{0}: \sigma_{b}^{2}=0$ sob o modelo (3.1) para um estudo balanceado, com 5 obervaçôes por unidade amostral, considerando distribuições assimétricas com caudas pesadas para o efeito aleatório e erro condicional.

\begin{tabular}{|c|c|c|c|c|c|c|c|c|c|c|c|}
\hline \multirow[b]{2}{*}{$\sigma_{b}^{2}$} & \multirow[b]{2}{*}{$\alpha$} & \multicolumn{2}{|c|}{$k=10$} & \multicolumn{2}{|c|}{$k=20$} & \multicolumn{2}{|c|}{$k=30$} & \multicolumn{2}{|c|}{$k=50$} & \multicolumn{2}{|c|}{$k=100$} \\
\hline & & $F$ & $J_{n}$ & $F$ & $J_{n}$ & $F$ & $J_{n}$ & $F$ & $J_{n}$ & $F$ & $J_{n}$ \\
\hline \multirow{3}{*}{0} & $1 \%$ & 0,8 & 1,4 & 1,1 & 1,3 & 1,5 & 1,3 & 1,7 & 1,3 & 1,7 & 1,2 \\
\hline & $5 \%$ & 4,4 & 5,7 & 4,4 & 5,6 & 4,3 & 5,4 & 4,4 & 5,3 & 4,6 & 5,2 \\
\hline & $10 \%$ & 7,2 & 11,1 & 7,0 & 10,8 & 7,5 & 10,6 & 7,9 & 10,5 & 8,3 & 10,3 \\
\hline \multirow{3}{*}{0.2} & $1 \%$ & 19,1 & 35,5 & 37,0 & 50,9 & 50,7 & 63,3 & 71,0 & 80,0 & 93,1 & 95,7 \\
\hline & $5 \%$ & 37,2 & 45,3 & 57,3 & 63,6 & 70,0 & 76,1 & 86,0 & 88,9 & 97,6 & 98,0 \\
\hline & $10 \%$ & 49,0 & 54,3 & 68,1 & 71,2 & 79,3 & 82,0 & 91,2 & 92,5 & 98,6 & 98,8 \\
\hline \multirow{3}{*}{0.5} & $1 \%$ & 52,5 & 68,0 & 78,4 & 85,4 & 90,1 & 93,8 & 97,7 & 98,7 & 99,9 & 100,0 \\
\hline & $5 \%$ & 69,9 & 76,5 & 89,1 & 91,0 & 95,8 & 96,7 & 99,1 & 99,4 & 100,0 & 100,0 \\
\hline & $10 \%$ & 78,3 & 81,2 & 93,0 & 93,3 & 97,5 & 97,8 & 99,5 & 99,7 & 100,0 & 100,0 \\
\hline \multirow{3}{*}{1} & $1 \%$ & 76,7 & 86,8 & 94,5 & 96,9 & 98,6 & 99,3 & 99,9 & 99,9 & 100,0 & 100,0 \\
\hline & $5 \%$ & 87,3 & 91,0 & 97,6 & 98,3 & 99,5 & 99,6 & 99,9 & 99,9 & 100,0 & 100,0 \\
\hline & $10 \%$ & 91,5 & 92,9 & 98,6 & 98,9 & 99,6 & 99,8 & 99,9 & 100,0 & 100,0 & 100,0 \\
\hline
\end{tabular}

mostra mais poderoso em todas as configurações, de forma similar às situações previamente analisadas.

A análise das tabelas 3.3-3.5 mostra indícios de que o teste $F$ é mais afetado pela falta de normalidade por parte dos efeitos aleatórios e erros condicionais do que pelo desbalanceamento. Além disso, o teste baseado na estatística $J_{n}$ se mostra mais poderoso, comparativamente com o teste $F$, em todas as situações consideradas, além de se mostrar menos sensivel ao desbalanceamento. Tais conclusões devem levar em consideração o aspecto liberal do teste proposto, principalmente para pequenas amostras. Para contornar este problema, podemos considerar métodos de reamostragem, como o bootstrap por exemplo, para obter a distribuição empírica da estatística $J_{n}$ sob $\mathcal{H}_{0}$ e utilizar o fato de que a hipótese deve ser rejeitada quando $J_{n}>c$, de forma a obter um teste "exato" para amostras de tamanho moderado. Esse é um assunto de interesse para futuras pesquisas. Detalhes a respeito do uso do método bootstrap para construir intervalos de confiança e testes de hipóteses, podem ser encontrados em Shao e Tu (1995), Davison e Hinkley (1997) e Lehmann e Romano (2005, Cap. 15), por exemplo. 


\subsection{Modelos com intercepto aleatório}

Nesta seção consideramos uma extensão do teste obtido anteriormente para modelos em que a média da variável resposta é modelada por variáveis explicativas, ou seja, modelos da forma

$$
Y_{i j}=\mathbf{x}_{i j}^{\top} \boldsymbol{\beta}+b_{i}+e_{i j}, \quad i=1, \ldots, k, \quad j=1, \ldots, n_{i}
$$

em que $\mathbf{x}_{i j}$ é um vetor de dimensão $p \times 1$ e $\boldsymbol{\beta}$ é um vetor de parâmetros $p \times 1$. Assim como na seção anterior, consideramos válidas as suposições (3.2)-(3.4) e que as observações e as linhas da matriz $\mathbf{X}$ estão ordenadas lexicograficamente. Nosso interesse é verificar se o intercepto aleatório deve ser incluído no modelo, ou seja, testar

$$
\mathcal{H}_{0}: \sigma_{b}^{2}=0 \text { versus } \mathcal{H}_{1}: \sigma_{b}^{2}>0
$$

É válido ressaltar, assim como na seção anterior, que o teste continua válido mesmo nas situações em que as variâncias dos efeitos aleatórios são diferentes.

Admitamos inicialmente que o vetor de parâmetros $\boldsymbol{\beta}$ e $\sigma_{e}^{2}$ são conhecidos, e definamos

$$
T_{n \beta}=T_{n}(\boldsymbol{\beta})=\sum_{1 \leq r<s \leq n} \eta_{n r s} \psi\left(Y_{r}, Y_{s}, \boldsymbol{\beta}\right)
$$

em que $\psi\left(Y_{r}, Y_{s}, \boldsymbol{\beta}\right)=\left(Y_{r}-\mathbf{x}_{r}^{\top} \boldsymbol{\beta}\right)\left(Y_{s}-\mathbf{x}_{s}^{\top} \boldsymbol{\beta}\right)$, com $\mathbf{x}_{s}^{\top}$ representando a s-ésima linha da matriz de especificação $\mathbf{X}$, correspondente ao modelo (3.23) expresso na forma geral (1.2), com os pesos $\eta_{n r s}$ definidos em (3.16). Pelo Teorema 3.1, temos que, sob $\mathcal{H}_{0}: \sigma_{b}^{2}=0$,

$$
J_{n \beta}=\frac{T_{n \beta}}{\sigma_{e}^{2} \sqrt{M_{n}}} \stackrel{\mathcal{D}}{\longrightarrow} \mathcal{N}(0,1)
$$

quando $k \rightarrow \infty$.

Nossa proposta consiste em mostrar que a convergência acima continua válida se substituirmos $\boldsymbol{\beta}$ e $\sigma_{e}^{2}$ por estimadores consistentes, nomeadamente, $\widehat{\beta}_{n}$ e $S_{n}$.

A seguir explicitamos algumas condições de regularidade que garantem a existência de um estimador consistente para o vetor de parâmetros $\boldsymbol{\beta}$. Para detalhes, sugerimos que o leitor consulte Sen e Singer (1993, Cap.7), por exemplo. Daqui em diante, adicionaremos um índice $n$ a todos os vetores e matrizes, de forma a evidenciar a dependência do tamanho da amostra.

Consideramos válidas as condições generalizadas de Noether:

i) $\max _{1 \leq k \leq n} \mathbf{x}_{n k}^{\top}\left(\mathbf{X}_{n}^{\top} \mathbf{X}_{n}\right)^{-1} \mathbf{x}_{n k} \rightarrow 0$, quando $n \rightarrow \infty$; 
ii) $\lim _{n \rightarrow \infty} n^{-1}\left(\mathbf{X}_{n}^{\top} \mathbf{X}_{n}\right)=\mathbf{A}$, sendo $\mathbf{A}$ uma matriz finita e positiva definida.

Utilizando o Teorema Limite Central de Hájek-Šidak (veja, por exemplo, Sen e Singer, 1993, p.119) podemos mostrar que o estimador de mínimos quadrados ordinários $\widehat{\boldsymbol{\beta}}_{n}=$ $\left(\mathbf{X}_{n}^{\top} \mathbf{X}_{n}\right)^{-1} \mathbf{X}_{n}^{\top} \mathbf{Y}_{n}$ é tal que

$$
\sqrt{n}\left(\widehat{\boldsymbol{\beta}}_{n}-\boldsymbol{\beta}\right) \stackrel{\mathcal{D}}{\longrightarrow} \mathcal{N}_{p}\left(0, \sigma_{e}^{2} \mathbf{A}^{-1}\right)
$$

ou equivalentemente, que

$$
\left(\mathbf{X}_{n}^{\top} \mathbf{X}_{n}\right)^{1 / 2}\left(\widehat{\boldsymbol{\beta}}_{n}-\boldsymbol{\beta}\right) \stackrel{\mathcal{D}}{\longrightarrow} \mathcal{N}_{p}\left(0, \sigma_{e}^{2} \mathbf{I}_{p}\right) .
$$

Além disso, é possível mostrar também que o estimador não viesado quadrático de norma mínima (MINQUE) (minimum norm quadratic unbiased estimator) de $\sigma_{e}^{2}$, a saber, $S_{n}=\mathbf{Y}_{n}^{\top}\left(\mathbf{I}_{n}-\mathbf{W}_{n}\left(\mathbf{W}_{n}^{\top} \mathbf{W}_{n}\right)^{-} \mathbf{W}_{n}^{\top}\right) \mathbf{Y}_{n} /\left(n-p^{*}\right) \stackrel{\mathbb{P}}{\longrightarrow} \sigma_{e}^{2}$, com $\mathbf{W}_{n}=\left(\mathbf{X}_{n}, \mathbf{Z}_{n}\right), \mathbf{M}^{-}$representando uma inversa generalizada da matriz $\mathbf{M}$ e $p^{*}=\operatorname{posto}\left(\mathbf{W}_{n}\right)$. Para detalhes, veja Apêndice B seção B.5. Utilizando o Teorema de Slutsky (veja, por exemplo, Sen e Singer, 1993, Teorema 3.4.2, p.127), podemos concluir que

$$
\frac{\sqrt{n}\left(\widehat{\boldsymbol{\beta}}_{n}-\boldsymbol{\beta}\right)}{\sqrt{S_{n}}} \stackrel{\mathcal{D}}{\longrightarrow} \mathcal{N}_{p}\left(0, \mathbf{A}^{-1}\right),
$$

ou equivalentemente, que

$$
\frac{\left(\mathbf{X}_{n}^{\top} \mathbf{X}_{n}\right)^{1 / 2}\left(\widehat{\boldsymbol{\beta}}_{n}-\boldsymbol{\beta}\right)}{\sqrt{S_{n}}} \stackrel{\mathcal{D}}{\longrightarrow} \mathcal{N}_{p}\left(0, \mathbf{I}_{p}\right)
$$

Consideremos agora a seguinte estatística

$$
T_{n \hat{\beta}}=\sum_{1 \leq r<s \leq n} \eta_{n r s} \psi\left(Y_{r}, Y_{s}, \widehat{\boldsymbol{\beta}}_{n}\right)
$$

que corresponde à estatística (3.25) com $\boldsymbol{\beta}$ substituído por $\widehat{\boldsymbol{\beta}}_{n}$ e seja

$$
J_{n \hat{\beta}}=\frac{T_{n \hat{\beta}}}{S_{n} \sqrt{M_{n}}} .
$$

O resultado a seguir, cuja demonstração está apresentada na Seção B.6 no Apêndice B, serve como base para definirmos um teste assintótico para a hipótese $\mathcal{H}_{0}: \sigma_{b}^{2}=0$ em modelos do tipo (3.23).

Teorema 3.3. Consideremos o modelo (3.23), a estatística $J_{n \hat{\beta}}$ definida em (3.28) e denotemos por $\mathbf{x}_{s}^{\top}$ a s-ésima linha da matriz X. Sob as suposições (3.2)-(3.4), admitindo que a seqüência $\left\{n_{i}\right\}_{i=1, \ldots, k}$ é limitada e que existe uma constante $\gamma<\infty$ tal que

$$
\sup _{1 \leq s \leq n}\left\|\mathbf{x}_{s}\right\| \leq \gamma
$$

Nobre, Juvêncio S. 
então sob $\mathcal{H}_{0}: \sigma_{b}^{2}=0$, temos

$$
J_{n \hat{\beta}} \stackrel{\mathcal{D}}{\longrightarrow} \mathcal{N}(0,1)
$$

quando $k \rightarrow \infty(\Rightarrow n \rightarrow \infty)$

Como, sob $\mathcal{H}_{1}, \mathbb{E}\left[J_{n \beta}\right]>0$ (para detalhes, veja Apêndice B Seção B.9) e usando o fato de que $J_{n \beta}-J_{n \hat{\beta}} \stackrel{\mathbb{P}}{\longrightarrow} 0$, então rejeitamos a hipótese nula ao nível de significância $\alpha \in(0,1)$ se $J_{n \hat{\beta}} \geq z_{\alpha}$, com $z_{\alpha}$ representando o quantil de ordem $(1-\alpha)$ da distribuição normal padrão.

Admitindo a existência do quarto momento da distribuição dos efeitos aleatórios, o teorema seguinte (que constitui uma extensão do Teorema 3.2 e cuja demonstração é apresentada no Apêndice B Seção B.7) fornece a distribuição assintótica da estatística de teste $J_{n \hat{\beta}}$ para uma seqüência de hipóteses locais de Pitman.

Teorema 3.4. Consideremos o modelo (3.23) sob a seqüência $\mathcal{H}_{1 n}: \sigma_{b}^{2}=\delta^{2} / \sqrt{n}$ e a estatística $J_{n \hat{\beta}}$ definida em (3.28). Denotando $\lim _{n \rightarrow \infty} M_{n} / n^{3}=\lambda$ e admitindo que $\mathbb{E}\left[b_{i}^{4}\right]<$ $\infty$, então, sob as hipóteses do Teorema 3.3 temos

$$
J_{n \hat{\beta}} \stackrel{\mathcal{D}}{\longrightarrow} \mathcal{N}\left(\frac{\delta^{2}}{2 \sigma_{e}^{2} \sqrt{\lambda}}, 1\right)
$$

quando $k \rightarrow \infty(\Rightarrow n \rightarrow \infty)$.

Esse resultado, indica que o poder do teste é uma função monótona estritamente crescente do coeficiente de correlação intra-classe $\rho=\delta^{2} /\left(\delta^{2}+\sigma_{e}^{2}\right)$. Comparando os Teoremas 3.1 e 3.2, com os Teoremas 3.3 e 3.4, respectivamente, concluímos que a inclusão de variáveis explicativas na parte funcional fixa do modelo não altera a distribuição assintótica da estatística de teste.

\subsubsection{Estudos de Simulação}

Para ilustrar o comportamento do teste em amostras pequenas e de tamanho moderado, repetimos o processo de simulação descrito na Subseção 3.2.1 sob o modelo

$$
Y_{i j}=1+4 x_{i j}+b_{i}+e_{i j}, \quad i=1, \ldots, k, \quad j=1, \ldots, n_{i},
$$

com as variáveis explicativas geradas (de forma independente) através de uma distribuição $U(0,2)$.

Em um primeiro momento, consideramos um estudo balanceado, geramos o erro condicional através de uma distribuição normal padrão e variamos apenas a distribuição do efeito aleatório; em particular, consideramos três situações: 
i) $b_{i} \sim \mathcal{N}\left(0, \sigma_{b}^{2}\right)$

ii) $b_{i} \sim t_{3} \times \sqrt{\sigma_{b}^{2} / 3}$;

iii) $b_{i} \sim\left(\left(Y_{1}-\mathbb{E}\left[Y_{1}\right]\right) / \sqrt{\operatorname{Var}\left[Y_{1}\right]}\right) \times \sigma_{b}$, em que $Y_{1}$ representa uma variável aleatória com distribuição $t$ assimétrica com parâmetro de localização 0 , parâmetro de dispersão 1 , com 4, 1 graus de liberdade e parâmetro de assimetria $\lambda=1$.

Os resultados estão dispostos na Tabela 3.6. Em todas as situações, utilizamos o estimador quadrático não viesado de norma mínima (veja Seção B.5 para detalhes) de $\sigma_{e}^{2}$ no denominador de (3.28). O poder empírico é praticamente o mesmo obtido quando utilizamos o estimador de máxima verossimilhança restrita de $\sigma_{e}^{2}$, derivado sob normalidade. Por esta razão, não apresentamos os resultados do estudo de simulação correspondente. Tal comportamento confirma empiricamente os resultados obtidos em Butler e Louis (1992) e Verbeke e Lesaffre (1996, 1997).

Com o objetivo de avaliar o comportamento do teste em situações em que o erro condicional não possui distribuição normal, repetimos o processo de simulação anterior, gerando a distribuição do erro condicional como: $e_{i j} \sim t_{5} \times \sqrt{3 / 5}$. Os resultados estão dispostos na Tabela 3.7.

Assim como nos casos previamente estudados, percebemos que o teste proposto apresenta uma característica liberal; entretanto, em amostras de tamanho moderado (com pelo menos 30 unidades amostrais e 5 observações por unidade amostral) o tamanho do teste é próximo do nível nominal. Quando simulamos a distribuição do erro condicional através de uma distribuição $t$ o teste proposto se mostra menos liberal comparativamente à situação em que simulamos da distribuição normal padrão. Em ambos os estudos, o poder empírico do teste tem um comportamento similar para as três distribuições adotadas para o efeito aleatório.

\subsubsection{Comparação entre os testes $F_{D}$ e $J_{n \widehat{\beta}}$}

Demidenko (2004) propõe um teste exato para verificar a significância conjunta de todos os candidatos a efeitos aleatórios no modelo (1.2)

$$
\mathbf{Y}=\mathbf{X} \boldsymbol{\beta}+\mathbf{Z b}+\mathbf{e}
$$

sob suposição de normalidade. A estatística do teste é

$$
F_{D}=\frac{\left(S_{\mathrm{MQO}}-S_{\min }\right) /\left(p^{*}-p\right)}{S_{\min } /\left(n-p^{*}\right)},
$$

Nobre, Juvêncio S. 
Tabela 3.6 Estimativas do poder do teste (\%) de $\mathcal{H}_{0}: \sigma_{b}^{2}=0$ sob o modelo (3.29) em estudos balanceados, sob normalidade do erro condicional.

\begin{tabular}{|c|c|c|c|c|c|c|c|c|c|c|c|c|c|}
\hline \multirow[b]{2}{*}{$\sigma_{b}^{2}$} & \multirow[b]{2}{*}{$\alpha$} & \multicolumn{4}{|c|}{$k=10$} & \multicolumn{4}{|c|}{$k=30$} & \multicolumn{4}{|c|}{$k=100$} \\
\hline & & $m=2$ & $m=4$ & $m=5$ & $m=10$ & $m=2$ & $m=4$ & $m=5$ & $m=10$ & $m=2$ & $m=4$ & $m=5$ & $m=10$ \\
\hline \multirow{3}{*}{0} & $1 \%$ & 6,9 & 4,9 & 4,2 & 2,6 & 3,7 & 3,0 & 2,7 & 1,8 & 2,2 & 2,0 & 1,4 & 1,1 \\
\hline & $5 \%$ & 11,3 & 8,3 & 8,1 & 6,8 & 8,6 & 7,6 & 7,5 & 6,1 & 7,0 & 6,1 & 5,4 & 5,2 \\
\hline & $10 \%$ & 16,3 & 13,7 & 12,7 & 11,6 & 13,6 & 12,1 & 11,7 & 10,5 & 12,5 & 11,1 & 10,6 & 10,4 \\
\hline \multicolumn{14}{|c|}{$b_{i} \sim \mathcal{N}\left(0, \sigma_{b}^{2}\right)$} \\
\hline \multirow{3}{*}{0.2} & $1 \%$ & 21,7 & 31,0 & 36,5 & 69,4 & 21,7 & 56,1 & 71,1 & 97,7 & 41,0 & 94,8 & 98,7 & 100,0 \\
\hline & $5 \%$ & 29,3 & 41,8 & 49,6 & 78,5 & 33,6 & 70,3 & 83,2 & 99,4 & 58,9 & 98,3 & 99,7 & 100,0 \\
\hline & $10 \%$ & 35,9 & 49,1 & 56,8 & 82,2 & 41,0 & 78,7 & 88,7 & 99,8 & 70,0 & 99,0 & 100,0 & 100,0 \\
\hline \multirow{3}{*}{0.5} & $1 \%$ & 37,2 & 66,9 & 75,0 & 93,9 & 58,6 & 96,8 & 98,0 & 100,0 & 94,2 & 100,0 & 100,0 & 100,0 \\
\hline & $5 \%$ & 48,5 & 75,9 & 82,9 & 96,0 & 72,7 & 98,4 & 99,6 & 100,0 & 98,4 & 100,0 & 100,0 & 100,0 \\
\hline & $0 \%$ & 54,0 & 80,5 & 87,1 & 97,3 & 79,9 & 99,1 & 100,0 & 100,0 & 99,1 & 100,0 & 100,0 & 100,0 \\
\hline \multirow{3}{*}{1.0} & $1 \%$ & 57,4 & 89,4 & 94,8 & 99,6 & 89,8 & 100,0 & 100,0 & 100,0 & 100,0 & 100,0 & 100,0 & 100,0 \\
\hline & $5 \%$ & 66,4 & 92,6 & 96,9 & 99,7 & 95,8 & 100,0 & 100,0 & 100,0 & 100,0 & 100,0 & 100,0 & 100,0 \\
\hline & $10 \%$ & 72,4 & 94,5 & 97,5 & 99,8 & 97,5 & 100,0 & 100,0 & 100,0 & 100,0 & 100,0 & 100,0 & 100,0 \\
\hline \multicolumn{14}{|c|}{$b_{i} \sim t_{3} \times\left(\sigma_{b}^{2} / 3\right)^{1 / 2}$} \\
\hline \multirow{3}{*}{0.2} & $1 \%$ & 20,3 & 29,3 & 35,0 & 59,0 & 21,5 & 43,8 & 56,1 & 85,4 & 38,9 & 83,7 & 93,6 & 99,9 \\
\hline & $5 \%$ & 27,1 & 40,3 & 47,4 & 69,7 & 33,5 & 56,7 & 70,0 & 91,7 & 57,1 & 92,2 & 97,7 & 100,0 \\
\hline & $10 \%$ & 33,2 & 47,1 & 55,1 & 76,1 & 41,4 & 66,2 & 76,2 & 93,9 & 67,4 & 95,3 & 98,9 & 100,0 \\
\hline \multirow{3}{*}{0.5} & $1 \%$ & 34,3 & 51,4 & 59,5 & 84,8 & 50,7 & 86,3 & 93,3 & 99,9 & 86,5 & 99,7 & 100,0 & 100,0 \\
\hline & $5 \%$ & 42,4 & 60,6 & 69,8 & 89,0 & 63,2 & 92,1 & 96,9 & 99,9 & 93,4 & 100,0 & 100,0 & 100,0 \\
\hline & $10 \%$ & 47,2 & 66,5 & 75,5 & 91,3 & 70,5 & 94,4 & 98,1 & 100,0 & 96,7 & 100,0 & 100,0 & 100,0 \\
\hline \multirow{3}{*}{1.0} & $1 \%$ & 51,1 & 74,7 & 85,1 & 96,4 & 76,4 & 97,8 & 99,5 & 100,0 & 99,4 & 100,0 & 100,0 & 100,0 \\
\hline & $5 \%$ & 61,0 & 82,2 & 89,7 & 97,8 & 84,8 & 98,6 & 99,9 & 100,0 & 99,8 & 100,0 & 100,0 & 100,0 \\
\hline & $10 \%$ & 68,0 & 86,7 & 92,3 & 98,5 & 89,4 & 99,4 & 100,0 & 100,0 & 100,0 & 100,0 & 100,0 & 100,0 \\
\hline \multicolumn{14}{|c|}{$b_{i} \sim t$-assimétrica } \\
\hline \multirow{3}{*}{0.2} & $1 \%$ & 20,8 & 32,5 & 38,6 & 56,7 & 24,2 & 52,3 & 61,8 & 89,7 & 40,3 & 88,9 & 95,8 & 100,0 \\
\hline & $5 \%$ & 28,9 & 42,4 & 49,6 & 66,7 & 36,5 & 64,9 & 74,7 & 94,2 & 59,7 & 94,1 & 98,2 & 100,0 \\
\hline & $10 \%$ & 35,2 & 49,1 & 56,4 & 72,3 & 46,2 & 72,0 & 80,0 & 95,4 & 69,5 & 96,8 & 99,1 & 100,0 \\
\hline \multirow{3}{*}{0.5} & $1 \%$ & 38,5 & 61,6 & 69,2 & 84,2 & 58,2 & 88,4 & 93,1 & 99,9 & 89,7 & 99,7 & 100,0 & 100,0 \\
\hline & $5 \%$ & 47,8 & 70,9 & 76,9 & 89,7 & 72,3 & 93,2 & 96,9 & 100,0 & 95,1 & 100,0 & 100,0 & 100,0 \\
\hline & $10 \%$ & 53,9 & 76,9 & 81,3 & 92,5 & 79,3 & 95,5 & 98,0 & 100,0 & 96,7 & 100,0 & 100,0 & 100,0 \\
\hline \multirow{3}{*}{1.0} & $1 \%$ & 56,9 & 86,2 & 86,2 & 97,5 & 85,9 & 98,3 & 99,0 & 100,0 & 98,9 & 100,0 & 100,0 & 100,0 \\
\hline & $5 \%$ & 68,3 & 89,3 & 89,4 & 97,6 & 91,1 & 99,3 & 100,0 & 100,0 & 100,0 & 100,0 & 100,0 & 100,0 \\
\hline & $10 \%$ & 72,7 & 92,6 & 92,6 & 98,6 & 93,4 & 99,4 & 100,0 & 100,0 & 100,0 & 100,0 & 100,0 & 100,0 \\
\hline
\end{tabular}

com

$$
S_{\mathrm{MQO}}=\|\mathbf{Y}-\mathbf{X} \widehat{\boldsymbol{\beta}}\|^{2}=\widehat{\mathbf{e}}^{\top} \widehat{\mathbf{e}}=\mathbf{Y}^{\top}\left(\mathbf{I}_{n}-\mathbf{P}_{\mathbf{X}}\right) \mathbf{Y}
$$

em que $\widehat{\boldsymbol{\beta}}=\left(\mathbf{X}^{\top} \mathbf{X}\right)^{-1} \mathbf{X}^{\top} \mathbf{Y}$ é o estimador de mínimos quadrados ordinários (MQO), $\widehat{\mathbf{e}}=$ $\mathbf{Y}-\mathbf{X} \widehat{\boldsymbol{\beta}}$ é o vetor de resíduos marginais e $\mathbf{P}_{\mathbf{X}}=\mathbf{X}\left(\mathbf{X}^{\top} \mathbf{X}\right)^{-1} \mathbf{X}^{\top}$ é a matriz de projeção de $\mathbf{Y}$ no espaço gerado pelas colunas de $\mathbf{X}$ e

$$
S_{\min }=\min _{\boldsymbol{\beta}^{*}}\left\|\mathbf{Y}-\mathbf{W} \boldsymbol{\beta}^{*}\right\|^{2}=\mathbf{Y}^{\top}\left(\mathbf{I}_{n}-\mathbf{P}_{\mathbf{W}}\right) \mathbf{Y}
$$

com $\mathbf{W}=(\mathbf{X}, \mathbf{Z})$, de dimensão $n \times(p+k q), \boldsymbol{\beta}^{*}=(\boldsymbol{\beta}, \mathbf{b})^{\top}$ e $\mathbf{P}_{\mathbf{W}}=\mathbf{W}\left(\mathbf{W}^{\top} \mathbf{W}\right)^{-} \mathbf{W}^{\top}$. Os 
Tabela 3.7 Estimativas do poder do teste (\%) de $\mathcal{H}_{0}: \sigma_{b}^{2}=0$ sob o modelo (3.29) em estudos balanceados, com $e_{i j} \sim t_{5} \times \sqrt{3 / 5}$.

\begin{tabular}{|c|c|c|c|c|c|c|c|c|c|c|c|c|c|}
\hline \multirow[b]{2}{*}{$\sigma_{b}^{2}$} & \multirow[b]{2}{*}{$\alpha$} & \multicolumn{4}{|c|}{$k=10$} & \multicolumn{4}{|c|}{$k=30$} & \multicolumn{4}{|c|}{$k=100$} \\
\hline & & $m=2$ & $m=4$ & $m=5$ & $m=10$ & $m=2$ & $m=4$ & $m=5$ & $m=10$ & $m=2$ & $m=4$ & $m=5$ & $m=10$ \\
\hline \multirow{2}{*}{0} & $1 \%$ & 5,8 & 3,6 & 3,5 & 2,6 & 3,2 & 2,8 & 2,2 & 1,8 & 2,0 & 1,4 & 1,1 & 1,1 \\
\hline & $10 \%$ & 15,8 & 12,4 & 11,2 & 10,9 & 12,9 & 11,5 & 11,1 & 10,4 & 10,7 & 10,6 & 10,5 & 10,3 \\
\hline \multicolumn{14}{|c|}{$b_{i} \sim \mathcal{N}\left(0, \sigma_{b}^{2}\right)$} \\
\hline \multirow[t]{2}{*}{0.2} & $5 \%$ & 28,6 & 41,2 & 53,0 & 78,1 & 36,5 & 73,1 & 81,7 & 98,9 & 60,1 & 96,7 & 99,6 & 100,0 \\
\hline & $10 \%$ & 35,6 & 49,5 & 60,3 & 83,6 & 44,8 & 79,3 & 86,9 & 98,9 & 69,9 & 98,5 & 99,8 & 100,0 \\
\hline \multirow{3}{*}{0.5} & $1 \%$ & 38,9 & 68,0 & 76,2 & 95,4 & 56,4 & 94,9 & 98,4 & 100,0 & 94,6 & 100,0 & 100,0 & 100,0 \\
\hline & $5 \%$ & 50,2 & 77,7 & 83,7 & 97,4 & 70,2 & 97,7 & 99,3 & 100,0 & 97,9 & 100,0 & 100,0 & 100,0 \\
\hline & $10 \%$ & 56,9 & 81,1 & 87,6 & 98,7 & 78,6 & 98,5 & 99,8 & 100,0 & 98,8 & 100,0 & 100,0 & 100,0 \\
\hline \multicolumn{14}{|c|}{$b_{i} \sim t_{3} \times\left(\sigma_{b}^{2} / 3\right)^{1 / 2}$} \\
\hline \multirow{3}{*}{0.2} & $1 \%$ & 15,8 & 27,6 & 34,2 & 54,7 & 23,4 & 46,5 & 58,2 & 89,6 & 40,0 & 85,4 & 94,7 & 99,9 \\
\hline & $5 \%$ & 23,8 & 36,1 & 44,6 & 65,1 & 36,4 & 59,7 & 70,4 & 94,3 & 57,3 & 92,7 & 98,0 & 100,0 \\
\hline & $10 \%$ & 29,8 & 41,7 & 51,4 & 72,4 & 45,2 & 67,2 & 76,6 & 96,4 & 66,9 & 95,8 & 98,9 & 100,0 \\
\hline \multirow{3}{*}{0.5} & $1 \%$ & 34,3 & 52,2 & 63,1 & 84,4 & 51,7 & 82,5 & 91,8 & 99,9 & 86,6 & 100,0 & 100,0 & 100,0 \\
\hline & $5 \%$ & 43,8 & 61,3 & 72,2 & 89,0 & 64,5 & 89,5 & 95,0 & 100,0 & 93,8 & 100,0 & 100,0 & 100,0 \\
\hline & $10 \%$ & 49,6 & 67,4 & 76,9 & 91,7 & 71,0 & 92,8 & 96,9 & 100,0 & 96,6 & 100,0 & 100,0 & 100,0 \\
\hline \multirow{3}{*}{1.0} & $1 \%$ & 50,4 & 77,1 & 82,0 & 96,7 & 77,4 & 98,1 & 99,3 & 100,0 & 99,0 & 100,0 & 100,0 & 100,0 \\
\hline & $5 \%$ & 61,9 & 84,7 & 87,0 & 98,0 & 86,2 & 99,5 & 99,7 & 100,0 & 99,7 & 100,0 & 100,0 & 100,0 \\
\hline & $10 \%$ & 67,0 & 87,9 & 90,6 & 98,6 & 90,3 & 99,8 & 99,9 & 100,0 & 99,9 & 100,0 & 100,0 & 100,0 \\
\hline 0.5 & $10 \%$ & 56,3 & 87,9 & 94,8 & 99,8 & 81,3 & 99,5 & 99,9 & 100,0 & 99,3 & 100,0 & 100,0 & 100,0 \\
\hline \multirow{3}{*}{1.0} & $1 \%$ & 69,0 & 96,4 & 98,9 & 100,0 & 92,9 & 100,0 & 100,0 & 100,0 & 100,0 & 100,0 & 100,0 & 100,0 \\
\hline & $5 \%$ & 78,6 & 98,0 & 99,9 & 100,0 & 97,3 & 100,0 & 100,0 & 100,0 & 100,0 & 100,0 & 100,0 & 100,0 \\
\hline & $10 \%$ & 83,0 & 98,7 & 100,0 & 100,0 & 98,1 & 100,0 & 100,0 & 100,0 & 100,0 & 100,0 & 100,0 & 100,0 \\
\hline
\end{tabular}

termos $S_{\mathrm{MQO}}$ e $S_{\min }$ representam, respectivamente, a soma de quadrados dos resíduos do modelo sem efeitos aleatórios e do modelo em que se considerem todos os efeitos como fixos. Por (3.31) e (3.32), podemos reescrever a estatística de teste (3.30) como

$$
F_{D}=\frac{\mathbf{Y}^{\top}\left(\mathbf{P}_{\mathbf{W}}-\mathbf{P}_{\mathbf{X}}\right) \mathbf{Y} /\left(p^{*}-p\right)}{\mathbf{Y}^{\top}\left(\mathbf{I}_{n}-\mathbf{P}_{\mathbf{X}}\right) \mathbf{Y} /\left(n-p^{*}\right)}
$$

Podemos mostrar (veja, Demidenko, 2004, p. 137), que sob $\mathcal{H}_{0}: \operatorname{Cov}(\mathbf{b})=\boldsymbol{\Omega}=\mathbf{0}, F_{D} \sim$ $F\left(p^{*}-p, n-p^{*}\right), \operatorname{com} p^{*}=\operatorname{posto}(\mathbf{W})$. Dada uma amostra oriunda de uma população para a qual o modelo (1.2) é aceitável, rejeita-se a hipótese de inexistência conjunta dos efeitos 
aleatórios se $F_{D}>F_{1-\alpha}\left(p^{*}-p, n-p^{*}\right)$, com $F_{1-\alpha}\left(p^{*}-p, n-p^{*}\right)$ representando o quantil de ordem $(1-\alpha)$ da distribuição $F\left(p^{*}-p, n-p^{*}\right)$.

Para comparar os dois testes em questão, repetimos o processo de simulação descrito na Subseção 3.2.2 sob o modelo modelo (3.29). Os resultados estão dispostos, respectivamente, nas Tabelas 3.8-3.11.

Tabela 3.8 Estimativas do poder do teste (\%) de $\mathcal{H}_{0}: \sigma_{b}^{2}=0$ no modelo (3.29) para um estudo balanceado, com 5 observações por unidade amostral, sob normalidade.

\begin{tabular}{rrrrrrrrrrrr}
\hline \hline & \multicolumn{1}{c}{$k=10$} & \multicolumn{2}{c}{$k=20$} & \multicolumn{2}{c}{$k=30$} & \multicolumn{2}{c}{$k=50$} & \multicolumn{2}{c}{$k=100$} \\
$\sigma_{b}^{2}$ & $\alpha$ & $F_{D}$ & $J_{n \hat{\beta}}$ & $F_{D}$ & $J_{n \hat{\beta}}$ & $F_{D}$ & $J_{n \hat{\beta}}$ & $F_{D}$ & $J_{n \hat{\beta}}$ & $F_{D}$ & $J_{n \hat{\beta}}$ \\
\hline \hline & $1 \%$ & 1,1 & 4,1 & 1,1 & 2,7 & 1,0 & 2,5 & 1,0 & 1,4 & 1,0 & 1,3 \\
0 & $5 \%$ & 5,3 & 8,1 & 4,8 & 7,5 & 4,9 & 7,3 & 5,0 & 5,8 & 4,7 & 5,3 \\
& $10 \%$ & 10,3 & 12,7 & 9,7 & 12,0 & 9,5 & 11,6 & 10,1 & 11,0 & 9,8 & 10,5 \\
\hline \hline & $1 \%$ & 20,0 & 38,0 & 38,6 & 57,9 & 55,8 & 69,3 & 79,4 & 86,9 & 97,9 & 99,1 \\
0.2 & $5 \%$ & 40,7 & 49,3 & 62,8 & 72,8 & 76,9 & 82,4 & 91,4 & 95,0 & 99,5 & 99,7 \\
& $10 \%$ & 53,5 & 59,0 & 74,6 & 80,3 & 84,3 & 88,8 & 94,1 & 97,6 & 99,9 & 100,0 \\
\hline \hline & $1 \%$ & 58,8 & 76,3 & 88,2 & 94,1 & 96,9 & 98,6 & 100,0 & 100,0 & 100,0 & 100,0 \\
0.5 & $5 \%$ & 77,9 & 84,0 & 95,5 & 97,4 & 99,2 & 99,6 & 100,0 & 100,0 & 100,0 & 100,0 \\
& $10 \%$ & 83,4 & 86,7 & 97,7 & 98,1 & 99,6 & 99,9 & 100,0 & 100,0 & 100,0 & 100,0 \\
\hline \hline & $1 \%$ & 88,2 & 93,7 & 99,3 & 99,7 & 100,0 & 100,0 & 100,0 & 100,0 & 100,0 & 100,0 \\
1 & $5 \%$ & 95,0 & 96,4 & 99,8 & 99,8 & 100,0 & 100,0 & 100,0 & 100,0 & 100,0 & 100,0 \\
& $10 \%$ & 96,0 & 97,4 & 99,9 & 100,0 & 100,0 & 100,0 & 100,0 & 100,0 & 100,0 & 100,0 \\
\hline \hline
\end{tabular}

O teste proposto se mostra levemente mais liberal comparativamente ao teste obtido no modelo de ANOVA com um único efeito aleatório, provavelmente pela necessidade de se obter um estimador consistente para $\boldsymbol{\beta}$. Uma possível justificativa é o fato de que o estimador de $\boldsymbol{\beta}$ pode apresentar um viés elevado em pequenas amostras. Nas quatro situações consideradas observamos um comportamento similar àquele detectado no estudo de simulação descrito na subseção 3.2.2; O teste $F_{D}$ (3.30) parece ser mais afetado pela falta de normalidade por parte dos efeitos aleatórios e/ou erros condicionais do que pelo desbalanceamento. Além disso, o teste baseado em $J_{n \widehat{\beta}}$ se mostra liberal, sendo este viés relativamente pequeno (a diferença absoluta entre o tamanho do teste e o nível de significância menor ou igual a 2,3\%) em amostras com 30 (ou mais) unidades amostrais, cada uma com um número não muito pequeno (maior ou igual a 5, por exemplo) de observações. O teste baseado na estatística $J_{n \widehat{\beta}}$ é mais poderoso do que o teste $F_{D}$, em todas as situações consideradas. Tais conclusões devem levar em consideração o aspecto liberal do teste proposto, principalmente para pequenas amostras. 
Tabela 3.9 Estimativas do poder do teste (\%) de $\mathcal{H}_{0}: \sigma_{b}^{2}=0$ no modelo (3.29) para um estudo desbalanceado, com o número de observações por unidade amostral sendo selecionado de forma equiprovável no conjunto $\{5,6,7,8,9,10\}$, com distribuições com caudas pesadas para o efeito aleatório e o erro condicional.

\begin{tabular}{|c|c|c|c|c|c|c|c|c|c|c|c|}
\hline \multirow[b]{2}{*}{$\sigma_{b}^{2}$} & \multirow[b]{2}{*}{$\alpha$} & \multicolumn{2}{|c|}{$k=10$} & \multicolumn{2}{|c|}{$k=20$} & \multicolumn{2}{|c|}{$k=30$} & \multicolumn{2}{|c|}{$k=50$} & \multicolumn{2}{|c|}{$k=100$} \\
\hline & & $F_{D}$ & $J_{n \hat{\beta}}$ & $F_{D}$ & $J_{n \hat{\beta}}$ & $F_{D}$ & $J_{n \hat{\beta}}$ & $F_{D}$ & $J_{n \hat{\beta}}$ & $F_{D}$ & $J_{n \hat{\beta}}$ \\
\hline \multirow{3}{*}{0} & $1 \%$ & 0,9 & 2,3 & 0,9 & 2,0 & 1,4 & 1,8 & 1,2 & 1,5 & 1,8 & 1,4 \\
\hline & $5 \%$ & 4,5 & 6,8 & 4,5 & 6,1 & 4,7 & 5,9 & 5,9 & 5,6 & 6,1 & 5,4 \\
\hline & $10 \%$ & 8,8 & 11,5 & 8,9 & 11,3 & 9,3 & 11,0 & 10,4 & 10,8 & 11,0 & 10,3 \\
\hline \multirow{3}{*}{0.2} & $1 \%$ & 32,6 & 48,6 & 48,8 & 66,8 & 62,5 & 77,1 & 79,8 & 86,6 & 90,6 & 99,7 \\
\hline & $5 \%$ & 51,1 & 59,5 & 68,4 & 78,0 & 78,5 & 83,3 & 89,1 & 95,2 & 95,1 & 99,9 \\
\hline & $10 \%$ & 61,7 & 67,2 & 77,7 & 82,5 & 84,9 & 89,6 & 93,1 & 98,3 & 97,4 & 100,0 \\
\hline \multirow{3}{*}{0.5} & $1 \%$ & 66,6 & 79,3 & 90,7 & 95,6 & 93,8 & 95,9 & 95,5 & 99,9 & 100,0 & 100,0 \\
\hline & $5 \%$ & 77,1 & 83,6 & 96,0 & 97,5 & 98,4 & 99,8 & 99,0 & 100,0 & 100,0 & 100,0 \\
\hline & $10 \%$ & 86,4 & 89,9 & 97,6 & 98,6 & 99,2 & 99,9 & 99,9 & 100,0 & 100,0 & 100,0 \\
\hline \multirow{3}{*}{1} & $1 \%$ & 82,7 & 91,5 & 91,4 & 96,0 & 94,8 & 96,2 & 99,3 & 100,0 & 100,0 & 100,0 \\
\hline & $5 \%$ & 87,7 & 92,4 & 96,4 & 97,9 & 99,9 & 100,0 & 100,0 & 100,0 & 100,0 & 100,0 \\
\hline & $10 \%$ & 89,5 & 95,5 & 96,7 & 99,0 & 100,0 & 100,0 & 100,0 & 100,0 & 100,0 & 100,0 \\
\hline
\end{tabular}

Tabela 3.10 Estimativas do poder do teste (\%) de $\mathcal{H}_{0}: \sigma_{b}^{2}=0$ no modelo (3.29) para um estudo desbalanceado, com o número de observações por unidade amostral sendo selecionado segundo uma distribuição $2+G(0.15)$, sob normalidade.

\begin{tabular}{|c|c|c|c|c|c|c|c|c|c|c|c|}
\hline \multirow[b]{2}{*}{$\sigma_{b}^{2}$} & \multirow[b]{2}{*}{$\alpha$} & \multicolumn{2}{|c|}{$k=10$} & \multicolumn{2}{|c|}{$k=20$} & \multicolumn{2}{|c|}{$k=30$} & \multicolumn{2}{|c|}{$k=50$} & \multicolumn{2}{|c|}{$k=100$} \\
\hline & & $F_{D}$ & $J_{n \hat{\beta}}$ & $F_{D}$ & $J_{n \hat{\beta}}$ & $F_{D}$ & $J_{n \hat{\beta}}$ & $F_{D}$ & $J_{n \hat{\beta}}$ & $F_{D}$ & $J_{n \hat{\beta}}$ \\
\hline \multirow{3}{*}{0} & $1 \%$ & 1,0 & 3,2 & 1,1 & 2,3 & 1,2 & 2,0 & 1,0 & 1,7 & 0,9 & 1,4 \\
\hline & $5 \%$ & 5,0 & 7,8 & 5,0 & 7,1 & 5,2 & 5,9 & 5,0 & 5,8 & 4,6 & 5,2 \\
\hline & $10 \%$ & 10,3 & 12,1 & 9,9 & 11,9 & 10,2 & 11,2 & 9,9 & 10,5 & 9,6 & 10,3 \\
\hline \multirow{3}{*}{0.2} & $1 \%$ & 22,4 & 50,2 & 50,1 & 69,2 & 82,3 & 89,2 & 92,7 & 98,0 & 99,4 & 100,0 \\
\hline & $5 \%$ & 42,9 & 62,3 & 71,1 & 81,1 & 89,1 & 95,2 & 97,8 & 99,2 & 100,0 & 100,0 \\
\hline & $10 \%$ & 55,6 & 69,6 & 80,1 & 86,0 & 95,1 & 97,1 & 99,0 & 99,5 & 100,0 & 100,0 \\
\hline \multirow{3}{*}{0.5} & $1 \%$ & 64,1 & 83,0 & 96,6 & 97,7 & 99,8 & 99,9 & 100,0 & 100,0 & 100,0 & 100,0 \\
\hline & $5 \%$ & 80,2 & 89,2 & 99,0 & 98,6 & 99,9 & 100,0 & 100,0 & 100,0 & 100,0 & 100,0 \\
\hline & $10 \%$ & 86,8 & 92,9 & 99,4 & 99,0 & 100,0 & 100,0 & 100,0 & 100,0 & 100,0 & 100,0 \\
\hline \multirow{3}{*}{1} & $1 \%$ & 93,9 & 96,8 & 99,7 & 100,0 & 100,0 & 100,0 & 100,0 & 100,0 & 100,0 & 100,0 \\
\hline & $5 \%$ & 97,5 & 97,7 & 99,9 & 100,0 & 100,0 & 100,0 & 100,0 & 100,0 & 100,0 & 100,0 \\
\hline & $10 \%$ & 98,4 & 98,6 & 99,9 & 100,0 & 100,0 & 100,0 & 100,0 & 100,0 & 100,0 & 100,0 \\
\hline
\end{tabular}

Nobre, Juvêncio S. 
Tabela 3.11 Estimativas do poder do teste (\%) de $\mathcal{H}_{0}: \sigma_{b}^{2}=0$ no modelo (3.1), para um estudo balanceado, com 5 obervações por unidade amostral, considerando distribuições assimétricas com caudas pesadas para o efeito aleatório e erro condicional.

\begin{tabular}{rrrrrrrrrrrr}
\hline \hline & \multicolumn{1}{c}{$k=10$} & \multicolumn{2}{c}{$k=20$} & \multicolumn{2}{c}{$k=30$} & \multicolumn{2}{c}{$k=50$} & \multicolumn{2}{c}{$k=100$} \\
$\sigma_{b}^{2}$ & $\alpha$ & $F_{D}$ & $J_{n \hat{\beta}}$ & $F_{D}$ & $J_{n \hat{\beta}}$ & $F_{D}$ & $J_{n \hat{\beta}}$ & $F_{D}$ & $J_{n \hat{\beta}}$ & $F_{D}$ & $J_{n \hat{\beta}}$ \\
\hline \hline & $1 \%$ & 0,8 & 3,1 & 1,1 & 2,3 & 1,3 & 1,8 & 1,5 & 1,6 & 1,7 & 1,3 \\
0 & $5 \%$ & 3,2 & 7,5 & 3,5 & 6,3 & 3,9 & 5,9 & 4,1 & 5,5 & 4,2 & 5,1 \\
& $10 \%$ & 7,7 & 12,6 & 8,0 & 11,1 & 7,9 & 11,1 & 8,1 & 10,9 & 8,0 & 10,4 \\
\hline \hline & $1 \%$ & 14,9 & 46,6 & 36,4 & 62,6 & 56,4 & 74,2 & 78,5 & 88,0 & 99,3 & 100,0 \\
0.2 & $5 \%$ & 37,4 & 60,0 & 64,7 & 76,9 & 78,7 & 86,1 & 92,6 & 96,1 & 100,0 & 100,0 \\
& $10 \%$ & 52,6 & 67,6 & 78,0 & 87,0 & 86,0 & 90,6 & 97,2 & 97,9 & 100,0 & 100,0 \\
\hline \hline & \multirow{2}{*}{$1 \%$} & 56,4 & 86,9 & 83,7 & 96,4 & 96,8 & 98,8 & 100,0 & 100,0 & 100,0 & 100,0 \\
0.5 & $5 \%$ & 80,7 & 92,8 & 90,6 & 97,8 & 99,0 & 99,5 & 100,0 & 100,0 & 100,0 & 100,0 \\
& $10 \%$ & 87,3 & 95,0 & 94,9 & 99,0 & 100,0 & 100,0 & 100,0 & 100,0 & 100,0 & 100,0 \\
\hline \hline & $1 \%$ & 87,3 & 98,4 & 96,3 & 99,6 & 100,0 & 100,0 & 100,0 & 100,0 & 100,0 & 100,0 \\
1 & $5 \%$ & 91,0 & 99,4 & 98,8 & 99,6 & 100,0 & 100,0 & 100,0 & 100,0 & 100,0 & 100,0 \\
& $10 \%$ & 93,5 & 99,7 & 100,0 & 100,0 & 100,0 & 100,0 & 100,0 & 100,0 & 100,0 & 100,0 \\
\hline \hline
\end{tabular}

\subsection{Modelos com intercepto e inclinação aleatórios}

Nesta seção desenvolvemos um teste assintótico para verificar a significância da componente de variância da inclinação aleatória na presença do intercepto aleatório, ou seja, para hipóteses da forma (1.7) versus (1.8) com $q=1$. Mesmo sob normalidade do vetor de erros condicionais e dos efeitos aleatórios, não existe teste exato, sendo necessário recorrer a algum teste assintótico, como o teste da razão de verossimilhanças generalizada ou o teste escore.

Consideremos o modelo

$$
Y_{i j}=\mathbf{x}_{i j}^{\top} \boldsymbol{\beta}+b_{i 0}+z_{i j} b_{i 1}+e_{i j}, \quad i=1, \ldots, k, \quad j=1, \ldots, n_{i},
$$

em que $\mathbf{x}_{i j}$ é um vetor de dimensão $p \times 1, \boldsymbol{\beta}$ é um vetor de parâmetros $p \times 1 \mathrm{e}$

$$
\begin{aligned}
& \mathbf{b}_{i}=\left(b_{i 0}, b_{i 1}\right)^{\top} \stackrel{\mathrm{iid}}{\sim}(\mathbf{0}, \boldsymbol{\Omega}), \\
& e_{i j} \stackrel{\mathrm{iid}}{\sim}\left(0, \sigma_{e}^{2}\right), \text { com } \mathbb{E}\left[e_{i j}^{4}\right]=\mu_{4 e}<\infty \\
& \mathbf{b}_{i} \text { e } e_{i j} \text { independentes }
\end{aligned}
$$

com

$$
\boldsymbol{\Omega}=\left[\begin{array}{cc}
\sigma_{0}^{2} & \sigma_{12} \\
\sigma_{12} & \sigma_{1}^{2}
\end{array}\right]
$$

Nobre, Juvêncio S. 
Como nas seções anteriores, consideramos que as observações e as linhas da matriz $\mathbf{X}$ estão ordenadas lexicograficamente. Suponhamos que o interesse é avaliar a necessidade de inclusão do efeito aleatório referente à inclinação, ou seja, testar

$$
\mathcal{H}_{0}: \sigma_{0}^{2}>0 \text { e } \sigma_{1}^{2}=0
$$

versus

$$
\mathcal{H}_{1}: \Omega \text { NND, com } \sigma_{0}^{2}>0 \text { e } \sigma_{1}^{2}>0
$$

Sob $\mathcal{H}_{0}$, o modelo (3.33) se reduz a

$$
\begin{aligned}
Y_{i j} & =\mathbf{x}_{i j}^{\top} \boldsymbol{\beta}+b_{i 0}+e_{i j} \\
& =\mathbf{x}_{i j}^{\top} \boldsymbol{\beta}+e_{i j}^{*},
\end{aligned}
$$

$\operatorname{com} e_{i j}^{*}=b_{i 0}+e_{i j}, i=1, \ldots, k, j=1, \ldots, n_{i}$ que pode ser reescrito como

$$
\mathbf{Y}=\mathbf{X} \boldsymbol{\beta}+\mathbf{e}^{*}
$$

em que $\mathbf{e}^{*} \sim(\mathbf{0}, \mathbf{V})$, com $\mathbf{V}=\bigoplus_{i=1}^{k} \mathbf{V}_{i}, \mathbf{V}_{i}=\sigma_{e}^{2} \mathbf{I}_{n_{i}}+\sigma_{0}^{2} \mathbf{J}_{n_{i}}$ e $\mathbf{J}_{n_{i}}=\mathbf{1}_{n_{i}} \mathbf{1}_{n_{i}}^{\top}$. Suponhamos que o vetor $\mathbf{e}^{*}$ seja uma transformação bijetiva de um vetor $\mathbf{u} \sim\left(\mathbf{0}, \mathbf{I}_{n}\right)$ cujas componentes são tais que $u_{1}, \ldots, u_{n} \stackrel{\text { iid }}{\sim}(0,1)$, ou seja, que o vetor $\mathbf{e}^{*}$ possa ser reescrito como

$$
\mathbf{e}^{*}=\mathbf{V}^{1 / 2} \mathbf{u}
$$

com $\mathbf{V}^{1 / 2}$ denotando uma raiz quadrada da matriz $\mathbf{V}$, ou seja, uma matriz tal que $\mathbf{V}=$ $\mathbf{V}^{1 / 2}\left(\mathbf{V}^{1 / 2}\right)^{\top}$. Como $\mathbf{V}$ é uma matriz simétrica positiva definida, é possível mostrar que $\mathbf{V}^{1 / 2}$, obtida via decomposição espectral, também é positiva definida e unicamente definida; para detalhes, veja Harville (1997, Cap.21), por exemplo. Pré-multiplicando (3.40) por $\mathbf{V}^{-1 / 2}$ obtemos

$$
\mathbf{Y}^{*}=\mathbf{X}^{*} \boldsymbol{\beta}+\mathbf{u}
$$

$\operatorname{com} \mathbf{Y}^{*}=\mathbf{V}^{-1 / 2} \mathbf{Y}$ e $\mathbf{X}^{*}=\mathbf{V}^{-1 / 2} \mathbf{X}$

Em um primeiro instante, consideremos $\mathbf{V}$ e $\boldsymbol{\beta}$ conhecidos e definamos

$$
T_{n \beta}^{*}=T_{n}^{*}(\boldsymbol{\beta})=\sum_{1 \leq r<s \leq n} \eta_{n r s} \psi\left(Y_{r}^{*}, Y_{s}^{*}, \boldsymbol{\beta}\right)
$$

em que $\psi\left(Y_{r}^{*}, Y_{s}^{*}, \boldsymbol{\beta}\right)=\left(Y_{r}^{*}-\mathbf{x}_{r}^{* \top} \boldsymbol{\beta}\right)\left(Y_{s}^{*}-\mathbf{x}_{s}^{* \top} \boldsymbol{\beta}\right)$, com $\mathbf{x}_{s}^{* \top}$ representando a $s$-ésima linha 
de $\mathbf{X}^{*}$ no modelo (3.41) e $\eta_{n r s}$ dado em (3.16). Então, pelo Teorema 3.1, temos que, sob $\mathcal{H}_{0}: \sigma_{0}^{2}>0$ e $\sigma_{1}^{2}=0$

$$
\begin{aligned}
J_{n \beta}^{*} & =\frac{T_{n \beta}^{*}}{\sqrt{M_{n}}} \eta_{n r s}\left(Y_{r}^{*}-\mathbf{x}_{r}^{* \top} \boldsymbol{\beta}\right)\left(Y_{s}^{*}-\mathbf{x}_{s}^{* \top} \boldsymbol{\beta}\right) \\
& =\frac{\sum_{1 \leq r<s \leq n} \eta_{n r s} u_{r} u_{s}}{\sqrt{M_{n}}} \\
& =\frac{1 \leq r<s \leq n}{\sqrt{M_{n}}} \stackrel{\mathcal{D}}{\longrightarrow} \mathcal{N}(0,1), \text { quando } k \rightarrow \infty .
\end{aligned}
$$

Notemos que se $\sigma_{0}^{2}=0$, então $\mathbf{V}=\sigma_{e}^{2} \mathbf{I}_{n}$ e a estatística (3.43) se reduz a (3.26). Daqui em diante, adicionaremos um índice $n$ a todos os vetores e matrizes, de forma a evidenciar a dependência do tamanho da amostra.

Como $\mathbf{V}_{n}=\mathbf{V}_{n}\left(\sigma_{e}^{2}, \sigma_{0}^{2}\right)$ e $\boldsymbol{\beta}$ não são conhecidos, vamos substituí-los por estimadores consistentes. Se $S_{n}$ e $S_{n}^{0}$ forem estimadores consistentes de $\sigma_{e}^{2}$ e $\sigma_{0}^{2}$, respectivamente, concluímos que $\widehat{\mathbf{V}}_{n}=\mathbf{V}_{n}\left(S_{n}, S_{n}^{0}\right)=S_{n} \mathbf{I}_{n}+S_{n}^{0} \bigoplus_{i=1}^{k} \mathbf{J}_{n_{i}}=\bigoplus_{i=1}^{k} \widehat{\mathbf{V}}_{i}$ é um estimador consistente de $\mathbf{V}_{n}$.

Consideremos agora o estimador de Aitken

$$
\widehat{\boldsymbol{\beta}}_{n}^{a}=\left(\mathbf{X}_{n}^{\top} \widehat{\mathbf{V}}_{n}^{-1} \mathbf{X}_{n}\right)^{-1} \mathbf{X}_{n}^{\top} \widehat{\mathbf{V}}_{n}^{-1} \mathbf{Y}_{n}
$$

e admitamos válidas as condições generalizadas de Noether

i) $\max _{1 \leq k \leq n} \mathbf{x}_{n k}^{* \top}\left(\mathbf{X}_{n}^{* \top} \mathbf{X}_{n}^{*}\right)^{-1} \mathbf{x}_{n k}^{*} \rightarrow 0$, quando $n \rightarrow \infty$;

ii) $\lim _{n \rightarrow \infty} n^{-1}\left(\mathbf{X}_{n}^{* \top} \mathbf{X}_{n}^{*}\right)=\mathbf{M}$, sendo $\mathbf{M}$ uma matriz finita e positiva definida.

Além disso, denotando os autovalores da matriz $\left(\mathbf{V}_{n} \widehat{\mathbf{V}}_{n}^{-1}-\mathbf{I}_{n}\right)$ por $\lambda_{1}, \ldots, \lambda_{n}$ e os da matriz $\left(\mathbf{X}_{n}^{\top} \mathbf{V}_{n} \mathbf{X}_{n}\left(\mathbf{X}_{n}^{\top} \widehat{\mathbf{V}}_{n} \mathbf{X}_{n}\right)^{-1}-\mathbf{I}_{n}\right)$ por $\lambda_{1}^{*}, \ldots, \lambda_{n}^{*}$, é possível provar (veja Apêndice B, Seção B.8) que

$$
\lambda_{i}=o_{p}(1) \quad \text { e } \quad \lambda_{i}^{*}=o_{p}(1) \text { para } i=1, \ldots, n .
$$

Então, pelas condições generalizadas de Noether e por (3.45) é possível provar (para detalhes, veja Sen e Singer, 1993, Cap.7) que

$$
\sqrt{n}\left(\widehat{\boldsymbol{\beta}}_{n}^{a}-\boldsymbol{\beta}\right) \stackrel{\mathcal{D}}{\longrightarrow} \mathcal{N}_{p}\left(\mathbf{0}, \mathbf{M}^{-1}\right) .
$$

Consideremos agora à estatística

$$
T_{n \hat{\beta}^{a}}^{*}=\sum_{1 \leq r<s \leq n} \eta_{n r s} \psi\left(\widetilde{Y}_{r}^{*}, \tilde{Y}_{s}^{*}, \widehat{\boldsymbol{\beta}}_{n}^{a}\right)
$$

Nobre, Juvêncio S. 
com $\widetilde{Y}_{r}^{*}$ representando a $r$-ésima observação do vetor $\widehat{\mathbf{V}}_{n}^{-1 / 2} \mathbf{Y}_{n}$. Assim, a estatística (3.46) corresponde à estatística (3.43), quando substituimos $\boldsymbol{\beta}$ por $\widehat{\boldsymbol{\beta}}_{n}^{a}$ e $\mathbf{V}_{n}$ por $\widehat{\mathbf{V}}_{n}$. Definamos também

$$
J_{n \hat{\beta}^{a}}^{*}=\frac{T_{n \hat{\beta}^{a}}^{*}}{\sqrt{M_{n}}} .
$$

O teorema a seguir, cuja demonstração está apresentada na Seção B.10 no Apêndice B, é útil para definir um teste assintótico para a inclusão da componente de variância da inclinação aleatória na presença do intercepto aleatório.

Teorema 3.5. Consideremos o modelo (3.41), a estatística $J_{n \hat{\beta}^{a}}^{*}$ definida em (3.47) e denotemos por $\mathbf{x}_{s}^{* \top}$ a s-ésima linha da matriz $\mathbf{X}^{*}$. Sob as suposições (3.34)-(3.36), admitindo que a seqüência $\left\{n_{i}\right\}_{i=1, \ldots, k}$ é limitada e que existe uma constante $\gamma<\infty$ tal que

$$
\sup _{1 \leq s \leq n}\left\|\mathbf{x}_{s}^{*}\right\| \leq \gamma
$$

então, sob $\mathcal{H}_{0}: \sigma_{0}^{2}>0$ e $\sigma_{1}^{2}=0$, temos

$$
J_{n \hat{\beta}^{a}}^{*} \stackrel{\mathcal{D}}{\longrightarrow} \mathcal{N}(0,1)
$$

quando $k \rightarrow \infty(\Rightarrow n \rightarrow \infty)$.

Podemos utilizar $J_{n \hat{\beta}^{a}}^{*}$ como estatística de teste para $\mathcal{H}_{0}: \sigma_{0}^{2}>0$ e $\sigma_{1}^{2}=0$ versus $\mathcal{H}_{1}$ dada em (3.39). Como $J_{n \beta}^{*}-J_{n \hat{\beta}^{a}}^{*} \stackrel{\mathbb{P}}{\longrightarrow} 0$, e sob $\mathcal{H}_{1}, \mathbb{E}\left[J_{n \beta}^{*}\right] \neq 0$ (para detalhes veja Apêndice B, Seção B.9). Então, rejeitamos a hipótese nula ao nível de significância $\alpha$, se $\left|J_{n \hat{\beta}^{a}}^{*}\right| \geq z_{\alpha / 2}$, com $z_{\alpha / 2}$ representando o quantil de ordem $(1-\alpha / 2)$ da distribuição normal padrão.

Se o interesse é testar a hipótese de inexistência simultânea de todos os candidatos a efeitos aleatórios, em modelos do tipo

$$
Y_{i j}=\mathbf{x}_{i j}^{\top} \boldsymbol{\beta}+b_{01}+\ldots+z_{q i j} b_{i q}+e_{i j}, \quad i=1, \ldots, k, \quad j=1, \ldots, n_{i},
$$

podemos usar a estatística $J_{n \hat{\beta}}$ dada em (3.28). Neste caso, a hipótese nula deve ser rejeitada quando $\left|J_{n \hat{\beta}}\right| \geq z_{\alpha / 2}$, dado que, sob a hipótese alternativa, assim como no caso discutido acima, $\mathbb{E}\left[J_{n \beta}\right]$ pode ser negativa.

O modelo (3.33) após a pré-multiplicação de ambos os membros pela matriz $\mathbf{V}_{n}^{-1 / 2}$ pode ser reescrito como

$$
Y_{i j}^{*}=\mathbf{x}_{i j}^{* \top} \boldsymbol{\beta}+z_{i j}^{*} b_{i 1}+u_{i j}, \quad i=1, \ldots, k, \quad j=1, \ldots, n_{i} .
$$

Nobre, Juvêncio S. 
Definamos

$$
K_{n}=\sum_{i=1}^{k}\left(\frac{n-n_{i}}{n_{i}-1}\right) \sum_{1 \leq j_{1}<j_{2} \leq n_{i}} z_{i j_{1}}^{*} z_{i j_{2}}^{*} \text { e } \kappa=\lim _{n \rightarrow \infty} \operatorname{Sinal}\left(K_{n}\right) \text {. }
$$

Assumindo que as variáveis explicativas $z_{i j}^{*}$ em (3.48) são limitadas, concluímos que $K_{n}=$ $O\left(n^{2}\right)$. Supondo a existência do quarto momento da distribuição dos efeitos aleatórios, o teorema seguinte (que constitui uma extensão dos Teoremas 3.2 e 3.4) estabelece a distribuição assintótica da estatística de teste $J_{n \hat{\beta}^{a}}^{*}$ para uma seqüência de hipóteses locais de Pitman, quando os efeitos aleatórios referentes ao intercepto e inclinação são considerados independentes. Esse teorema pode ser estendido para a situação em que os efeitos são dependentes, bastando impor condições de regularidade adicionais.

Teorema 3.6. Consideremos o modelo (3.41) sob a seqüência $\mathcal{H}_{1 n}: \Omega \quad N N D$, com $\sigma_{0}^{2}>$ 0 e $\sigma_{1}^{2}=\delta^{2} / \sqrt{n}$, a estatística $J_{n \hat{\beta}^{a}}^{*}$ definida em (3.47), $\mathbf{x}_{s}^{* \top}$ a s-ésima linha da matriz $\mathbf{X}^{*}, z_{s}^{*}$ como definida em (3.48) e $\lim _{n \rightarrow \infty} \sqrt{n M_{n}} /\left|K_{n}\right|=\sqrt{\lambda^{*}}$. Sob as hipóteses do Teorema 3.5 e supondo adicionalmente que $\mathbb{E}\left[b_{i}^{4}\right]<\infty$ e que existe uma constante $\gamma_{2}<\infty$ tal que

$$
\sup _{1 \leq s \leq n}\left\|z_{s}^{*}\right\| \leq \gamma_{2}
$$

então

$$
J_{n \hat{\beta}^{a}}^{*} \stackrel{\mathcal{D}}{\longrightarrow} \mathcal{N}\left(\kappa \frac{\delta^{2}}{\sqrt{\lambda^{*}}}, 1\right)
$$

quando $k \rightarrow \infty(\Rightarrow n \rightarrow \infty)$.

Se $\mathbf{V}=\sigma_{e}^{2} \mathbf{I}_{n}$ e $z_{i j}=1$ para quaisquer $i, j$, implicando que $\kappa=1$ e que $\sqrt{\lambda^{*}}=2 \sqrt{\lambda}$, com $\lambda=\lim _{n \rightarrow \infty} M_{n} / n^{3}$, então o Teorema 3.6 se reduz ao Teorema 3.4. A demonstração está apresentada na Seção B.11 no Apêndice B.

O teste em questão pode ser naturalmente estendido para hipóteses mais gerais, como (1.7) versus (1.8), desde que seja possível obter estimativas consistentes para as componentes de variância (matriz V). Por exemplo, o MINQUE, discutido na Seção B.5 é consistente para $\sigma_{e}^{2}$ sob o modelo em questão. Com relação a $\sigma_{0}^{2}$ (ou de uma forma mais geral, para os elementos da matriz de covariâncias dos efeitos aleatórios, $\boldsymbol{\Omega}$ ), existem algumas propostas para obtenção de estimadores consistentes. Uma alternativa é considerar o MINQUE iterativo (IMINQUE) de $\sigma_{0}^{2}$ (ou de uma forma mais geral, dos elementos de $\Omega$ ), que é consistente, conforme discutido, por exemplo, em Brown (1976) e em Jiang (2007); uma segunda alternativa é considerar o estimador obtido pelo método dos momentos simulados 
proposto por Jiang (1998). Demidenko (2004, Caps. 4 e 5) apresenta uma série de estimadores consistentes para a matriz de covariâncias dos efeitos aleatórios, $\Omega$. Outras propostas são apresentadas em Jiang $(1997,2007)$ e Vonesh et al. (2002), por exemplo. Alternativas baseadas em procedimentos robustos (Jurečková e Sen, 1996) para obtenção de estimadores para as componentes de variância estão disponíveis na literatura; veja por exemplo, Pendergast e Broffitt (1985), Fellner (1986), Huggins (1993), Richardson e Welsh (1995), Mehrotra (1997), Welsh e Richardson (1997), Jiang e Zhang (2001), Müller e Uhlig (2001), Demidenko e Stukel (2002), Yau e Kuk (2002), Sinha (2004) e Yang et al. (2005).

\subsubsection{Estudo de simulação}

Apresentamos um breve estudo de simulação para ilustrar o comportamento do teste baseado na estatística $J_{n \widehat{\beta}^{a}}^{*}$ definida em (3.47) em amostras pequenas e moderadas e também para comparar a sua eficiência com aquela do teste da razão de verossimilhanças generalizada (obtido sob a suposição de normalidade).

Repetimos o processo de simulação descrito nas subseções anteriores sob o modelo

$$
Y_{i j}=1+4 x_{i j}+b_{i 0}+z_{i j} b_{i 1}+e_{i j}, \quad i=1, \ldots, k, j=1, \ldots, m
$$

com $e_{i j} \sim \mathcal{N}(0,1)$ e $\sigma_{0}^{2}=1$. As variáveis $x_{i j}$ e $z_{i j}$ foram geradas através de distribuições $U(0,2)$ independentes.

Em um primeiro estudo, simulamos a distribuição dos efeitos aleatórios $\mathbf{b}_{i}=\left(b_{i 0}, b_{i 1}\right)^{\top}$ com distribuição normal bivariada com vetor de médias $\mathbf{0}$ e matriz de covariâncias

$$
\boldsymbol{\Omega}=\left[\begin{array}{cc}
1 & 0.5 \sigma_{1} \\
0.5 \sigma_{1} & \sigma_{1}^{2}
\end{array}\right]
$$

de tal forma que $\operatorname{Corr}\left(b_{i 0}, b_{i 1}\right)=0.5 \mathbb{1}\left\{\sigma_{1}^{2}>0\right\}$. Os resultados estão dispostos na Tabela $3.12^{3}$. Utilizamos os estimadores obtidos pelo método de máxima verossimilhança restrita para implementação do teste proposto. Ao contrário de todas as situações já estudadas, tanto o teste proposto quanto o teste da razão de verossimilhanças generalizada (RVG) se mostram conservadores. Comparativamente, o teste da RVG se mostra menos conservador em amostras de tamanho pequeno; por outro lado, em amostras de tamanho moderado ou grande (e.g., $k \geq 30$ ) o teste proposto se mostra menos viesado além de apresentar um

${ }^{3}$ Neste caso, a distribuição assintótica do teste da razão de verossimilhanças é $\frac{1}{2} \chi_{1}^{2}+\frac{1}{2} \chi_{2}^{2}$. Uma tabela com os quantis de misturas de distribuições qui-quadrado centrais pode ser encontrada, por exemplo, no sítio http://www.ebi.ac.uk/goldman/publs/chi2bar.table.html 
maior poder na maioria das situações. Uma possível justificativa é o fato dos estimadores de MVR tenderem a superestimar $\sigma_{e}^{2}$ e $\sigma_{0}^{2}$. O comportamento do teste da RVG é análogo àquele observado nas simulações apresentadas em Morrell (1998).

Tabela 3.12 Estimativas do poder do teste (\%) de $\mathcal{H}_{0}: \sigma_{1}^{2}=0$ e $\sigma_{0}^{2}>0$ no modelo (3.50) para um estudo balanceado, com 5 observações por unidade amostral, sob normalidade.

\begin{tabular}{llrrrrrrrrrr}
\hline \hline & \multicolumn{1}{c}{$k=10$} & \multicolumn{2}{c}{$k=20$} & \multicolumn{2}{c}{$k=30$} & \multicolumn{2}{c}{$k=50$} & \multicolumn{2}{c}{$k=100$} \\
$\sigma_{b}^{2}$ & $\alpha$ & $T_{R V G}$ & $J_{n \hat{\beta}^{a}}^{*}$ & $T_{R V G}$ & $J_{n \hat{\beta}^{a}}^{*}$ & $T_{R V G}$ & $J_{n \hat{\beta}^{a}}^{*}$ & $T_{R V G}$ & $J_{n \hat{\beta}^{a}}^{*}$ & $T_{R V G}$ & $J_{n \hat{\beta}^{a}}^{*}$ \\
\hline \hline & $1 \%$ & 0,6 & 0,4 & 0,6 & 0,5 & 0,6 & 0,7 & 0,8 & 0,8 & 0,9 & 1,2 \\
0 & $5 \%$ & 3,5 & 2,3 & 3,7 & 3,4 & 3,8 & 4,0 & 4,3 & 4,4 & 4,6 & 4,9 \\
& $10 \%$ & 7,0 & 5,3 & 7,4 & 7,2 & 7,6 & 8,0 & 7,8 & 8,9 & 9,0 & 9,7 \\
\hline \hline & \multirow{2}{*}{$1 \%$} & 3,5 & 5,3 & 5,6 & 6,5 & 6,9 & 8,2 & 12,4 & 16,8 & 22,4 & 29,9 \\
0.2 & $5 \%$ & 9,5 & 13,4 & 11,6 & 16,4 & 13,5 & 18,6 & 20,8 & 27,8 & 40,3 & 39,1 \\
& $10 \%$ & 13,8 & 18,9 & 16,5 & 22,1 & 21,6 & 26,7 & 29,8 & 34,2 & 52,2 & 54,5 \\
\hline \hline & $1 \%$ & 7,1 & 11,6 & 13,3 & 18,0 & 20,0 & 24,3 & 26,6 & 24,3 & 41,8 & 44,3 \\
0.5 & $5 \%$ & 19,9 & 23,5 & 31,5 & 34,5 & 37,6 & 38,4 & 48,7 & 45,9 & 66,7 & 70,4 \\
& $10 \%$ & 28,6 & 34,5 & 44,3 & 48,2 & 49,3 & 52,4 & 62,1 & 61,3 & 79,8 & 84,0 \\
\hline \hline & $1 \%$ & 18,1 & 19,0 & 26,8 & 29,2 & 32,1 & 32,1 & 36,7 & 43,4 & 68,1 & 71,3 \\
& \multirow{2}{*}{$1 \% \%$} & 37,7 & 38,7 & 47,1 & 50,1 & 54,5 & 53,4 & 61,7 & 65,3 & 86,3 & 88,3 \\
& $10 \%$ & 52,8 & 51,3 & 58,7 & 62,2 & 65,6 & 64,5 & 72,1 & 75,7 & 92,8 & 94,5 \\
\hline \hline
\end{tabular}

Para ilustrar a sensibilidade do teste da RVG (obtido sob normalidade) quando a distribuição dos efeitos aleatórios não é normal, repetimos o processo de simulação, considerando que $b_{i t}=B_{t} \sigma_{t}(t=0,1)$, com $B_{0}$ e $B_{1}$ representando variáveis aleatórias iid com distribuição $t$ assimétrica com 4,1 graus de liberdade com média zero, variância igual a 1 e coeficiente de assimetria aproximadamente igual a 0,95 (correspondendo ao parâmetro de assimetria $\lambda \approx 0,5)$. O teste da RVG foi obtido sob normalidade; utilizamos estimadores de máxima verossimilhança restrita na implementação do teste proposto (3.47) (que continuam consistentes). Os resultados estão dispostos na Tabela 3.13. ${ }^{4}$

Comparativamente à situação anterior, ambos os testes se mostram conservadores, sendo o teste da RVG mais afetado com relação à má especificação da distribuição dos efeitos aleatórios. O teste proposto é menos conservador que o teste da RVG em todas as situações, além de se mostrar mais poderoso na maioria delas. Este exemplo ilustra bem o que pode ocorrer (perda de eficiência e poder) quando utilizamos indiscriminadamente o teste implementado na maioria dos software padrão (SAS, S, R,por exemplo) obtido usando a teoria da

${ }^{4}$ A distribuição assintótica do teste da razão de verossimilhanças, nesta configuração, é $\frac{1}{2} \chi_{0}^{2}+\frac{1}{2} \chi_{1}^{2}$. 
Tabela 3.13 Estimativas do poder do teste (\%) de $\mathcal{H}_{0}: \sigma_{1}^{2}=0$ e $\sigma_{0}^{2}>0$ no modelo (3.50) para um estudo balanceado, com 5 observações por unidade amostral, considerando distribuições assimétricas com caudas pesadas independentes para os efeitos aleatórios.

\begin{tabular}{cccccccccccc}
\hline \hline & \multicolumn{1}{c}{$k=10$} & \multicolumn{2}{c}{$k=20$} & \multicolumn{2}{c}{$k=30$} & \multicolumn{2}{c}{$k=50$} & \multicolumn{2}{c}{$k=100$} \\
$\sigma_{b}^{2}$ & $\alpha$ & $T_{R V G}$ & $J_{n \hat{\beta}^{a}}^{*}$ & $T_{R V G}$ & $J_{n \hat{\beta}^{a}}^{*}$ & $T_{R V G}$ & $J_{n \hat{\beta}^{a}}^{*}$ & $T_{R V G}$ & $J_{n \hat{\beta}^{a}}^{*}$ & $T_{R V G}$ & $J_{n \hat{\beta}^{a}}^{*}$ \\
\hline \hline & $1 \%$ & 0,1 & 0,4 & 0,4 & 0,4 & 0,4 & 0,7 & 0,7 & 0,8 & 1,4 & 0,9 \\
0 & $5 \%$ & 2,1 & 2,2 & 2,3 & 2,7 & 3,1 & 3,8 & 3,5 & 4,0 & 4,0 & 4,7 \\
& $10 \%$ & 5,5 & 5,5 & 5,8 & 7,0 & 6,3 & 7,7 & 8,0 & 8,5 & 8,9 & 9,4 \\
\hline \hline & $1 \%$ & 0,6 & 2,2 & 3,4 & 5,0 & 5,6 & 7,2 & 12,0 & 13,9 & 15,5 & 24,4 \\
0.2 & $5 \%$ & 3,4 & 7,0 & 8,2 & 12,3 & 10,8 & 15,9 & 18,7 & 22,0 & 31,4 & 34,4 \\
& $10 \%$ & 6,9 & 11,1 & 12,2 & 16,7 & 15,8 & 19,4 & 26,7 & 32,4 & 40,6 & 47,5 \\
\hline \hline & $1 \%$ & 3,3 & 4,8 & 7,4 & 8,9 & 10,2 & 12,1 & 16,4 & 19,2 & 31,9 & 37,5 \\
0.5 & $5 \%$ & 6,7 & 12,7 & 11,2 & 16,8 & 18,9 & 21,4 & 27,2 & 33,1 & 45,1 & 57,5 \\
& $10 \%$ & 11,1 & 16,6 & 16,8 & 25,3 & 24,3 & 29,4 & 34,7 & 43,1 & 57,9 & 72,4 \\
\hline \hline & $1 \%$ & 8,8 & 10,9 & 16,2 & 18,4 & 22,1 & 22,7 & 40,2 & 40,3 & 52,1 & 59,0 \\
& $5 \%$ & 17,5 & 18,8 & 26,2 & 30,2 & 39,6 & 43,4 & 56,3 & 58,2 & 70,2 & 79,5 \\
& $10 \%$ & 24,1 & 29,7 & 35,2 & 41,6 & 48,1 & 53,4 & 65,7 & 71,5 & 79,4 & 87,7 \\
\hline \hline
\end{tabular}

verossimilhança sob a suposição de normalidade, sem nos preocupar com a plausibilidade desta suposição. Um estudo de simulação mais amplo seria interessante para verificar o comportamento dos dois testes em questão, bem como do teste escore, quando variamos sensível ou abruptamente as suposições de normalidade ou de esfericidade das fontes de variação do modelo. Este também um tema de interesse para pesquisas futuras. 


\section{Capítulo 4}

\section{Aplicações}

Aqui aplicamos os testes propostos na análise dos dos exemplos apresentados no Capítulo 1.

\subsection{Análise do Exemplo 1.1}

Considerando as características discutidas em Singer et al. (2004), que analisaram outro conjunto de dados referente ao estudo mencionado no Exemplo 1.1, adotamos o modelo

$$
\ln y_{i j d}=\alpha_{j d}+\beta_{j d} \ln x_{i j d}+b_{i}+e_{i j d}
$$

$i=1, \ldots, 32, d=1,2,3,4$ e $j=0,1$, em que $y_{i j d}\left(x_{i j d}\right)$ é o índice de placa bacteriana pós-tratamento (pré-tratamento) relativo à $i$-ésima criança avaliada na $d$-ésima sessão de avaliação com à $j$-ésima escova $(j=0$ para escova convencional e $j=1$ para escova monobloco), $\alpha_{j d}$ é o efeito (fixo) associado à $j$-ésima escova na $d$-ésima sessão de avaliação, $\beta_{j d}$ é um coeficiente de uniformidade da taxa de placa residual esperada, $b_{i} \sim \mathcal{N}\left(0, \tau^{2}\right)$ e $e_{i j d} \sim \mathcal{N}\left(0, \sigma^{2}\right)$ são variáveis aleatórias independentes, correspondendo, respectivamente, aos efeitos aleatórios de criança e aos erros aleatórios.

A estratégia de análise utilizada (veja detalhes em Nobre, 2004 e Nobre e Singer, 2007), para simplificar o modelo saturado (4.1) consistiu em

(i) testar a homogeneidade entre os coeficientes de uniformidade para as duas escovas nas quatro sessões de avaliação, ou seja, verificar se $\beta_{j d}=\beta, j=0,1, d=1, \ldots, 4$ ou, em outras palavras reduzir o modelo (4.1) para

$$
\ln y_{i j d}=\alpha_{j d}+\beta \ln x_{i j d}+b_{i}+e_{i j d}
$$

(ii) testar a significância da interação e dos efeitos principais dos tipos de escova com relação aos coeficientes de placa bacteriana residual, ou seja, testar

$$
\alpha_{01}-\alpha_{11}=\alpha_{02}-\alpha_{12}=\alpha_{03}-\alpha_{13}=\alpha_{04}-\alpha_{14}
$$


$\mathrm{e}$

$$
\alpha_{j d}=\alpha_{j}, \quad d=1,2,3,4, \quad j=0,1
$$

(iii) ajustar o modelo que incorpora as conclusões obtidas em (i) e (ii), ou seja, reduzir o modelo (4.2) para

$$
\ln y_{i j d}=\alpha_{j}+\beta \ln x_{i j d}+b_{i}+e_{i j d}
$$

Detalhes podem ser encontrados em Nobre e Singer (2007). Nobre (2004) e Nobre e Singer (2007) apresentam análises de diagnóstico para o ajuste do modelo em questão. Eles concluem que os dados referentes às crianças \#12 e \#29 são potencialmente influentes com relação aos parâmetros de covariância do modelo (4.3). Na Tabela 4.1 apresentamos o impacto percentual nas estimativas dos parâmetros do modelo (4.3) da eliminação dessas crianças no ajuste do modelo. Quando removemos a criança \#29 da análise, a estimativa de máxima verossimilhança restrita de $\tau^{2}$ é bem próxima do seu respectivo erro-padrão estimado, deixando-nos céticos com relação à eventual significância de $\tau^{2}$.

Na Tabela 4.2 apresentamos os testes ${ }^{1}$ para componentes de variância, baseados nas estatísticas $J_{n \hat{\beta}_{n}}$, dada em (3.28), no teste exato $F_{D}$ dado em (3.30) e no teste da razão de verossimilhanças generalizada $\left(T_{R V G}\right)^{2}$, no modelo (4.3), obtidos com e sem as crianças \#12 e \#29. Em todos os testes, com exceção da situação em que removemos a criança \# 29 da análise, a componente de variância é significativa com nível de significância 5\%. Todavia, como existem mudanças inferencias quando retiramos a criança \#29, o modelo (4.3) não é robusto com relação à presença/ausência da criança \#29. Esta análise, mais uma vez, evidencia o fato de que a criança \#29 apresenta um peso desproporcional no ajuste do modelo em questão.

Fixando o nível de significância em 5\%, as conclusões baseadas no teste exato $F$ obtido sob suposição de normalidade ou no teste da razão de verossimilhanças generalizada (RVG) ou no teste baseado na estatística (3.28) são equivalentes; indicando que apesar do número de unidades amostrais $(k=32)$ não ser grande e da aparente normalidade do vetor de erros condicionais, o teste baseado na estatística (3.28) se comporta bem, comparativamente, aos testes exato e da RVG. Adicionalmente, se considerarmos um nível de significância igual

${ }^{1}$ Como não existem indícios contra a suposição de normalidade do erro condicional, veja Nobre e Singer (2007), utilizamos a estimativa de MVR de $\sigma_{e}^{2}$ no quociente de (3.28).

2 Obtido sob suposição de normalidade, cuja respectiva distribuição assintótica, sob a hipótese nula, é dada por $1 / 2 \chi_{0}^{2}+1 / 2 \chi_{1}^{2}$. 
a 1\%, tanto quando utilizamos o teste baseado na estatística (3.28) quanto o da RVG, concluímos que a componente de variância também não é significativa quando retiramos as crianças \#29 e \#12.

Os testes baseados na estatística (3.28) e o da RVG fornecem o mesmo resultado, se considerarmos os níveis de significância de 1\%, $5 \%$ ou 10\%, sendo que o primeiro apresenta a vantagem de não ser obtido sob a suposição de uma distribuição específica para as fontes de variação do modelo.

Tabela 4.1 Estimativas ( \pm erros-padrão estimados) dos parâmetros e impacto percentual do modelo (4.3) removendo as crianças \#12 and \#29.

\begin{tabular}{llllll}
\hline \hline Parâmetros & $\alpha_{0}$ & $\alpha_{1}$ & $\beta$ & $\tau^{2}$ & $\sigma^{2}$ \\
\hline \hline Completo & $-0,32 \pm 0,03$ & $-0,21 \pm 0,03$ & $1,06 \pm 0,06$ & $0,00634 \pm 0,0028$ & $0,021 \pm 0,02$ \\
Criança \#12 removida & $-0,32 \pm 0,03$ & $-0,22 \pm 0,03$ & $1,06 \pm 0,06$ & $0,00691 \pm 0,0027$ & $0,015 \pm 0,02$ \\
& $(0,0 \%)$ & $(-4,8 \%)$ & $(0,0 \%)$ & $(-9,0 \%)$ & $(\mathbf{2 8 , 6} \%)$ \\
Criança \#29 removida & $-0,33 \pm 0,03$ & $-0,19 \pm 0,03$ & $1,07 \pm 0,05$ & $0,00148 \pm 0,0013$ & $0,017 \pm 0,02$ \\
& $(0,0 \%)$ & $(9,5 \%)$ & $(0,9 \%)$ & $(\mathbf{7 6 , 7} \%)$ & $(-19,1 \%)$ \\
Criança \#12 e \#29 & $-0,32 \pm 0,03$ & $-0,19 \pm 0,03$ & $1,07 \pm 0,05$ & $0,00299 \pm 0,0014$ & $0,012 \pm 0,01$ \\
removidas & $(0,0 \%)$ & $(9,5 \%)$ & $(0,9 \%)$ & $\mathbf{( 5 2 , 8} \%)$ & $(\mathbf{4 2 , 9} \%)$ \\
\hline \hline
\end{tabular}

Tabela 4.2 Testes para componentes de variância no modelo (4.3) removendo as crianças \#12 e \#29.

\begin{tabular}{lrrrrrr}
\hline \hline Dados & $J_{n \hat{\beta}}$ & valor $p$ & $F_{D}$ & valor $p$ & $T_{R V G}$ & valor $p$ \\
\hline \hline Completo & 2,04 & 0,0206 & 2,40 & 0,0007 & 8,03 & 0,0023 \\
Removendo \#12 & 2,54 & 0,0055 & 14,45 & $<0,0001$ & 15,34 & $<0,0001$ \\
Removendo \#29 & 1,09 & $\mathbf{0 , 1 3 7 9}$ & 1,41 & $\mathbf{0 , 1 1 2 1}$ & 0,83 & $\mathbf{0 , 1 8 1 9}$ \\
Removendo \#12 e \#29 & 1,74 & 0,0413 & 1,98 & 0,0083 & 4,77 & 0,0145 \\
\hline \hline
\end{tabular}




\subsection{Análise do Exemplo 1.2}

Consideremos aqui os dados do Eexemplo 1.2. O número total de observações é $n=149$, com $\kappa=0.7557$, evidenciando um moderado grau de desbalanceamento.

Com base nos perfis individuais e médio apresentados na Figura 1.2, nossa estratégia de análise consistiu em ajustar um polinômio de segundo grau para representar a variação da resposta média populacional com o tempo e definir o grau do polinômio a ser ajustado aos perfis individuais (i.e., testar a inclusão dos efeitos aleatórios de intercepto e inclinação), ou seja, consideramos modelos do tipo

$$
y_{i j}=\alpha+\sum_{w=1}^{2} \beta_{w} t_{i j}^{w *}+b_{0 i}+b_{1 i} t_{i j}^{*}+e_{i j}, \quad i=1,2, \ldots, 32,
$$

em que $y_{i j}$ é o diâmetro sistólico da aorta por unidade de peso $(\mathrm{mm} / \mathrm{kg})$ relativo ao $i$-ésimo recém-nascido pré-termo com peso pequeno para a idade gestacional na $j$-ésima semana após concepção $(j=27, \ldots, 40), t_{i j}^{*}$ representa o tempo (em semanas após-concepção) centrados na média (33 semanas), $b_{0 i}$ e $b_{1 i}$ denotam os efeitos aleatórios de intercepto e inclinação e $e_{i j}$ representa o erro aleatório associado à observação $y_{i j}$.

Inicialmente supomos que $e_{i j} \stackrel{\text { iid }}{\sim} \mathcal{N}\left(0, \sigma_{e}^{2}\right)$ e que $\mathbf{b}_{i}=\left(b_{i 0}, b_{i 1}\right)^{\top} \stackrel{\text { iid }}{\sim} \mathcal{N}_{2}(\mathbf{0}, \boldsymbol{\Omega})(i=1, \ldots, 32)$, com $\Omega$ representando uma matriz de covariâncias não-estruturada. Para verificar a plausibilidade da suposição de normalidade, ajustamos o modelo "completo" (4.4) por meio do método de máxima verossimilhança restrita e utilizamos os procedimentos gráficos sugeridos em Hilden-Minton (1995), Nobre (2004) e Nobre e Singer (2007). Nas Figuras 4.1 e 4.2 , respectivamente, apresentamos os histogramas dos BLUP empíricos e o gráfico de envelope simulado e histograma para os resíduos condicionais com confundimento mínimo. Pela Figura 4.1 temos indícios de que o afastamento da normalidade é mais "acentuado" para o intercepto aleatório, apresentando inclusive uma leve assimetria à direita. Pela Figura 4.2 temos indício de que a distribuição dos erros condicionais apresenta caudas mais leves que as da distribuição normal. Por conseguinte, a utilização de testes para componentes de variância obtidos sob normalidade (ou sob suposição de simetria) para as fontes de variação do modelo, como, o teste da razão de verossimilhanças ou o teste escore, podem não ser adequados. Para este fim, sugerimos utilizar os testes assintóticos desenvolvidos nesta tese, que prescindem da especificação da distribuição das fontes de variação, evitando assim a necessidade de se modelar o erro condicional com uma distribuição com cauda mais leve que da normal e os efeitos aleatórios com distribuições assimétricas, por exemplo.

Na Tabela 4.3, apresentamos os resultados dos testes para componentes de variância baseados nas estatísticas (3.28) e (3.47). Os valores $J_{1}$ e $J_{2}$ correspondem respectivamente, 
Figura 4.1 Histograma para EBLUP padronizado.
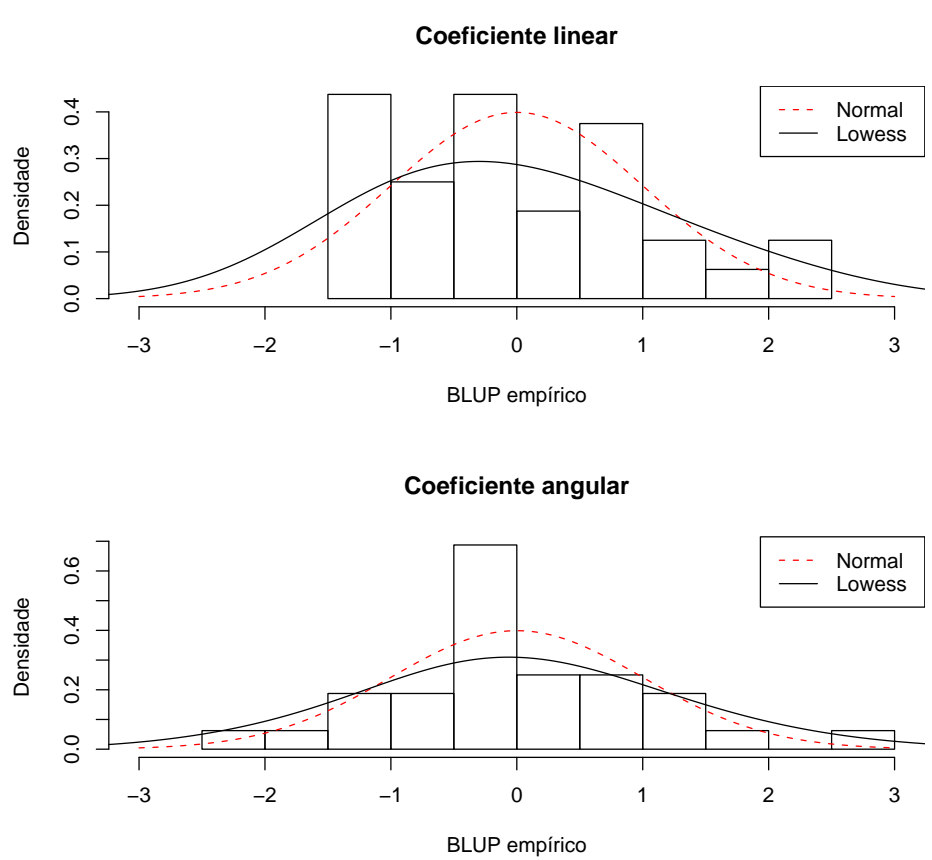

Figura 4.2 Envelope simulado com 95\% e histograma para o resíduo com confundimento mínimo padronizado.
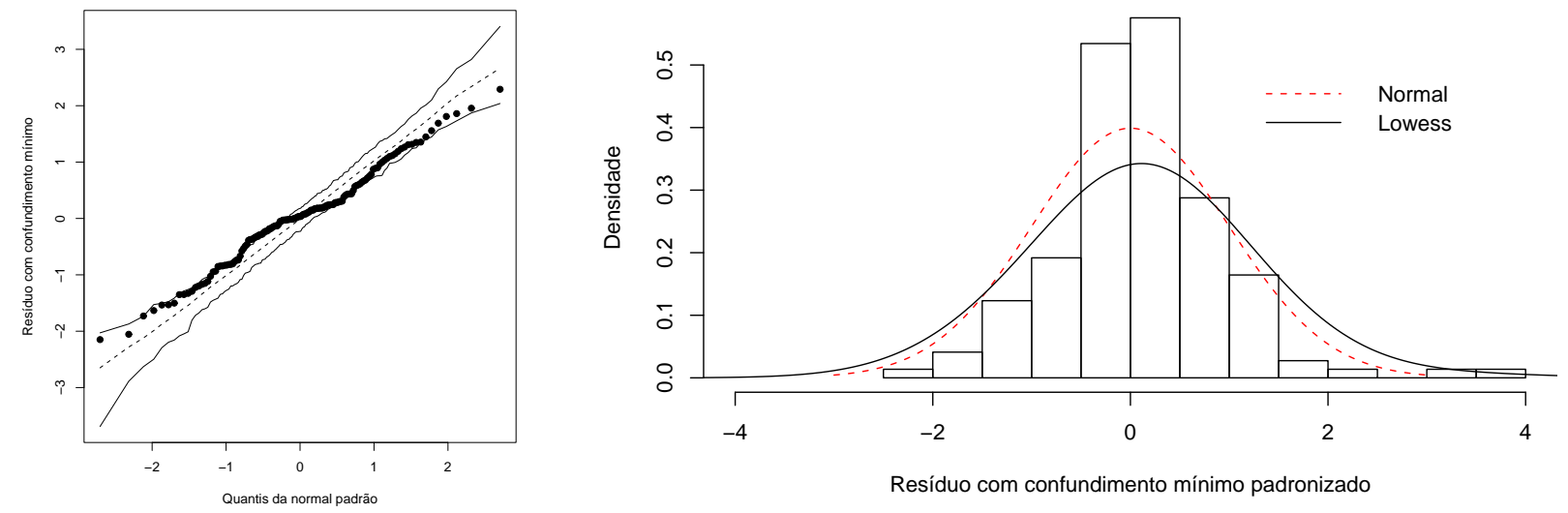

aos valores dos respectivos testes, quando utilizamos as estimativas das componentes de variâncias obtidas pelo método de máxima verossimilhança restrita (sob normalidade) e quando utilizamos o MINQUE de $\sigma_{e}^{2}$ e o estimador obtido pelo método dos momentos, (veja, e.g., Demidenko, 2004, Cap. 3) da matriz $\Omega$; neste caso não é necessário fazer suposições sobre a forma das distribuições, apenas sobre a existência dos momentos. 
Tabela 4.3 Testes para componentes de variância no modelo (4.4).

\begin{tabular}{llrrrr}
\hline \hline & Hipótese & $J_{1}$ & valor $p$ & $J_{2}$ & valor $p$ \\
\hline \hline (i) $\quad \mathcal{H}_{0}: \sigma_{0}^{2}=0$ & 27,26 & $<0,0001$ & 43,46 & $<0,0001$ \\
& $\mathcal{H}_{1}: \sigma_{0}^{2}>0$ & & & & \\
(ii) $\quad \mathcal{H}_{0}: \sigma_{0}^{2}>0$ e $\sigma_{1}^{2}=0$ & & & & & \\
& $\mathcal{H}_{1}: \Omega$ é NND, com $\sigma_{0}^{2}>0$ e $\sigma_{1}^{2}>0$ & & & & \\
&
\end{tabular}

As estimativas dos parâmetros do modelo, obtidas pelos dois métodos estão apresentadas na Tabela 4.4. Consideramos o estimador de Aitken para $\boldsymbol{\beta}$.

Tabela 4.4 Estimativas dos parâmetros do modelo (4.4).

\begin{tabular}{lrr}
\hline \hline Parâmetro & MVR & MINQUE e MM \\
\hline \hline$\alpha$ & 7,06 & 7,06 \\
$\beta_{1}$ & $-0,34$ & $-0,35$ \\
$\beta_{2}$ & $-0,02$ & $-0,01$ \\
$\sigma_{e}^{2}$ & 0,35 & 0,55 \\
$\sigma_{0}^{2}$ & 0,81 & 0,27 \\
$\sigma_{01}$ & $<0,01$ & 0,01 \\
$\sigma_{1}^{2}$ & 0,03 & 0,15 \\
\hline \hline
\end{tabular}

Por fim, concluímos que tanto o intercepto aleatório como a inclinação aleatória são altamente significativos. Rocha e Singer (2007) usando métodos descritivos apresentados em Rocha (2004), também selecionam intercepto e inclinação aleatórios. 


\section{Capítulo 5}

\section{Considerações finais}

\subsection{Conclusões}

Neste trabalho propomos testes para componentes de variância em modelos lineares mistos baseados em decomposições de estatísticas $U$. As principais vantagens apresentadas da nossa proposta são:

i) Não é necessário especificar completamente o modelo probabilístico em questão; em especial supomos apenas a existência do quarto momento do erro condicional e do segundo momento dos efeitos aleatórios. O resultado é aplicável a uma classe muito ampla de modelos lineares mistos.

ii) Adicionalmente, supondo a existência do quarto momento da distribuição dos efeitos aleatórios, encontramos a distribuição assintótica da estatística de teste sob seqüências de hipóteses alternativas locais.

iii) Não há necessidade de expansões, logo o teste não é afetado pela possível localização do parâmetro na fronteira do espaço paramétrico, ao contrário dos testes baseados na teoria da verossimilhança (razão de verossimilhanças e escore).

iv) O teste pode ser estendido para hipóteses bem gerais, como (1.7) versus (1.8), desde que seja possível obter estimativas consistentes das componentes de variância. A distribuição assintótica da estatística de teste é normal padrão, independentemente do número de componentes de variância que pretendemos testar, ao contrário dos testes da RVG e escore, cuja distribuição assintótica não é obtida de forma simples, quando o número de componentes de variância a serem testadas aumenta.

Comparamos a eficiência dos testes propostos com os testes exatos (obtidos sob normalidade) e com o teste da razão de verossimilhanças generalizada (obtido sob normalidade, na situação em que não existe teste exato) através de estudos de simulação. As principais características observadas na simulação seguem: 
i) Os testes propostos se comportaram bem em amostras de tamanho moderado em todas as situações consideradas (no que diz respeito ao seu tamanho estar próximo do nível nominal) além de se mostrarem mais poderosos que os testes exatos e o da razão de verossimilhanças em praticamente todas as situações.

ii) Tanto os testes exatos como o da RVG se apresentaram mais viesados do que o teste proposto, quando geramos as fontes de variação segundo distribuições diferentes da normal, mesmo em amostras de tamanho pequeno.

Pelos itens i) e ii) acima, concluímos que a utilização dos testes propostos se mostra mais adequada em situações em que a amostra é de tamanho moderado ou grande, independentemente da distribuição das fontes de variação ou em situações em que existem fortes afastamentos da normalidade.

Por fim, foi desenvolvida uma função em linguagem R (R Development Core Team, 2006) para calcular as estatísticas de testes (3.21), (3.28) e (3.47). A função supracitada, bem como os programas de simulação utilizados podem ser solicitados diretamente via email: juvencio@ime.usp.br.

\subsection{Pesquisas futuras}

Trabalhos futuros, oriundos da tese envolvem:

i) Desenvolvimento de um teste bootstrap, com o objetivo de diminuir o viés em amostras de tamanho pequeno.

ii) Um estudo de simulação mais amplo, com o objetivo de comparar os testes propostos com os testes da RVG e escore quando consideramos diferentes distribuições para o erro condicional e os efeitos aleatórios.

iii) Verificar a eficiência dos testes propostos quando utilizamos os diferentes estimadores consistentes propostos na literatura.

iv) Estender os testes para modelos não lineares mistos, modelos lineares generalizados mistos e modelos semi-paramétricos mistos.

vii) Estender os testes para modelos com erros nas variáveis (como considerado em Lin e Carrol, 1999). 


\section{Resultados básicos}

Definição A.1. Consideremos $\left\{X_{n}\right\}_{n \geq 1}$ uma seqüência de variáveis aleatórias. A seqüência é dita infinitesimal se $\forall \varepsilon>0$,

$$
\lim _{n \rightarrow \infty} \max _{1 \leq k \leq n} \mathbb{P}\left[\left|X_{n k}\right| \geq \varepsilon\right]=0
$$

Teorema A.1. (Teorema Limite Central para Martingais) Consideremos uma seqüência $\left\{X_{k}\right\}_{k \geq 1}$ de variáveis aleatórias tais que $\mathbb{E}\left[X_{k}\right]=0$ e $\mathbb{E}\left[X_{k}^{2}\right]=\sigma_{k}^{2}$ e $\mathbb{E}\left[X_{k} \mid X_{1}, \ldots, X_{k-1}\right]=0$ $\left(X_{0}=0\right)$. Seja $T_{n}=\sum_{k=1}^{n} X_{k}, S_{n}^{2}=\sum_{k=1}^{n} \sigma_{k}^{2}, v_{k}^{2}=\mathbb{E}\left[X_{k}^{2} \mid X_{1}, \ldots, X_{k-1}\right]$ e $w_{n}^{2}=\sum_{k=1}^{n} v_{k}^{2}$. Então,

i) $w_{n}^{2} / S_{n}^{2} \stackrel{\mathbb{P}}{\longrightarrow} 1$;

ii) $\forall \epsilon>0, \lim _{n \rightarrow \infty} S_{n}^{-2} \sum_{k=1}^{n} \mathbb{E}\left[X_{k}^{2} \mathbb{1}\left\{\left|X_{k}\right|>\epsilon S_{n}\right\}\right] \rightarrow 0$ (condição de Lindeberg);

a seqüência $\left\{X_{k}\right\}_{k \geq 1}$ é infinitesimal e

$$
Z_{n}=\frac{T_{n}}{S_{n}} \stackrel{\mathcal{D}}{\longrightarrow} \mathcal{N}(0,1)
$$

Para detalhes e exemplos, veja Dvoretzky (1972) e Sen e Singer (1993, p.120).

Definição A.2. (Método de Projeção) Considere $T_{n}$ uma estatística. A projeção de $T_{n}$ com relação às variáveis aleatórias $Y_{1}, \ldots, Y_{k_{n}}$ é dada por

$$
\check{T}_{n}=\mathbb{E}\left[T_{n}\right]+\sum_{i=1}^{k_{n}}\left(\mathbb{E}\left[T_{n} \mid Y_{i}\right]-\mathbb{E}\left[T_{n}\right]\right)
$$

Este método é também conhecido na literatura como projeção de Hájek. Para detalhes a respeito de outros métodos de projeção, veja Van der Vaart (1998, Cap.11). 


\author{
Apêndice B
}

\title{
Demonstrações
}

\section{B.1 Expressão (3.15)}

Em função de (3.10) e (3.14), e lembrando que $\sum_{i^{\prime} \neq i} n_{i^{\prime}}=n-n_{i}$, podemos reescrever a estatística $B_{n}$ como

$$
\begin{aligned}
\left(\begin{array}{c}
n \\
2
\end{array}\right) B_{n} & =\sum_{1 \leq i<i^{\prime} \leq k} \frac{n_{i} n_{i^{\prime}}}{2}\left\{2 U_{i i^{\prime}}-U_{i}-U_{i^{\prime}}\right\} \\
& =\left\{\sum_{1 \leq i<i^{\prime} \leq k} \frac{n_{i} n_{i^{\prime}}}{2}\left[\left(\begin{array}{c}
n_{i} \\
2
\end{array}\right) \sum_{1 \leq j<j^{\prime} \leq n_{i}} \psi\left(Y_{i j}, Y_{i j^{\prime}}\right)+\left(\begin{array}{c}
n_{i^{\prime}} \\
2
\end{array}\right)^{-1} \sum_{1 \leq k<k^{\prime} \leq n_{i^{\prime}}} \psi\left(Y_{i^{\prime} k}, Y_{i^{\prime} k^{\prime}}\right)\right]\right. \\
& \left.-\sum_{1 \leq i<i^{\prime} \leq k} \sum_{j=1}^{n_{i}} \sum_{j^{\prime}=1}^{n_{i^{\prime}}} \psi\left(Y_{i j}, Y_{i^{\prime} j^{\prime}}\right)\right\} \\
& =\left\{\sum_{i \neq i^{\prime}} \frac{n_{i} n_{i^{\prime}}}{2}\left(\begin{array}{c}
n_{i} \\
2
\end{array}\right)^{-1} \sum_{1 \leq j<j^{\prime} \leq n_{i}} \psi\left(Y_{i j}, Y_{i j^{\prime}}\right)-\sum_{1 \leq i<i^{\prime} \leq k} \sum_{j=1}^{n_{i}} \sum_{j^{\prime}=1}^{n_{i^{\prime}}} \psi\left(Y_{i j}, Y_{i^{\prime} j^{\prime}}\right)\right\} \\
& =\left\{\sum_{i \neq i^{\prime}} \frac{n_{i^{\prime}}}{n_{i}-1} \sum_{1 \leq j<j^{\prime} \leq n_{i}} \psi\left(Y_{i j}, Y_{i j^{\prime}}\right)-\sum_{1 \leq i<i^{\prime} \leq k} \sum_{j=1}^{n_{i^{\prime}}} \psi\left(Y_{j^{\prime}=1}, Y_{i j}, Y_{i^{\prime} j^{\prime}}\right)\right\} \\
= & \left\{\sum_{i=1}^{k} \frac{1}{n_{i}-1} \sum_{1 \leq j<j^{\prime} \leq n_{i}} \psi\left(Y_{i j}, Y_{i j^{\prime}}\right) \sum_{i^{\prime} \neq i} n_{i^{\prime}}-\sum_{1 \leq i<i^{\prime} \leq k} \sum_{j=1}^{n_{i^{\prime}}} \sum_{j^{\prime}=1} \psi\left(Y_{i j}, Y_{i^{\prime} j^{\prime}}\right)\right\} \\
= & \left\{\sum_{i=1}^{k}\left(\frac{n-n_{i}}{n_{i}-1} \sum_{1 \leq j<j^{\prime} \leq n_{i}} \psi\left(Y_{i j}, Y_{i j^{\prime}}\right)+\sum_{1 \leq i<i^{\prime} \leq k} \sum_{j=1}^{n_{i^{\prime}}}(-1) \psi\left(Y_{j^{\prime}=1}, Y_{i^{\prime} j^{\prime}}\right)\right\}\right. \\
& \sum_{1 \leq r<s \leq n} \eta_{n r s} \psi\left(Y_{r}, Y_{s}\right), \quad
\end{aligned}
$$

com $\eta_{\text {nrs }}$ definido em (3.16). 


\section{B.2 Identidades (3.17) e (3.18)}

Notemos que

$$
\begin{aligned}
\sum_{1 \leq r<s \leq n} \eta_{n r s} & =\sum_{i=1}^{k}\left(\frac{n-n_{i}}{n_{i}-1}\right)\left(\begin{array}{c}
n_{i} \\
2
\end{array}\right)+\sum_{1 \leq i<i^{\prime} \leq k}(-1) n_{i} n_{i^{\prime}} \\
& =\frac{1}{2} \sum_{i=1}^{k} n_{i}\left(n-n_{i}\right)-\sum_{1 \leq i<i^{\prime} \leq k} n_{i} n_{i^{\prime}} \\
& =\frac{1}{2} \sum_{i=1}^{k} n_{i} \sum_{i^{\prime} \neq i} n_{i^{\prime}}-\sum_{1 \leq i<i^{\prime} \leq k} n_{i} n_{i^{\prime}} \\
& =\frac{1}{2} \sum_{i \neq i^{\prime}} n_{i} n_{i^{\prime}}-\sum_{1 \leq i<i^{\prime} \leq k} n_{i} n_{i^{\prime}} \\
& =\sum_{1 \leq i<i^{\prime} \leq k} n_{i} n_{i^{\prime}}-\sum_{1 \leq i<i^{\prime} \leq k} n_{i} n_{i^{\prime}}=0
\end{aligned}
$$

o que prova (3.17). Por outro lado, também temos que

$$
\begin{aligned}
M_{n}=\sum_{1 \leq r<s \leq n} \eta_{n r s}^{2} & =\sum_{i=1}^{k}\left(\frac{n-n_{i}}{n_{i}-1}\right)^{2}\left(\begin{array}{c}
n_{i} \\
2
\end{array}\right)+\sum_{1 \leq i<i^{\prime} \leq k}(-1)^{2} n_{i} n_{i^{\prime}} \\
& =\frac{1}{2} \sum_{i=1}^{k} \frac{\left(n-n_{i}\right)^{2}}{\left(n_{i}-1\right)} n_{i}+\sum_{1 \leq i<i^{\prime} \leq k} n_{i} n_{i^{\prime}} ;
\end{aligned}
$$

Como $\frac{n_{i}}{n_{i}-1}=\left\{1+\frac{1}{n_{i}-1}\right\}$, temos

$$
\begin{aligned}
\sum_{i=1}^{k} \frac{\left(n-n_{i}\right)^{2}}{\left(n_{i}-1\right)} n_{i} & =\sum_{i=1}^{k}\left(n-n_{i}\right)\left\{1+\frac{1}{n_{i}-1}\right\}\left(n-n_{i}\right) \\
& =\sum_{i=1}^{k}\left(n-n_{i}\right)\left\{1+\frac{1}{n_{i}-1}\right\} \sum_{i^{\prime} \neq i} n_{i^{\prime}} \\
& =\sum_{i=1}^{k} \sum_{i^{\prime} \neq i}\left(n-n_{i}\right) n_{i^{\prime}}+\sum_{i=1}^{k} \sum_{i^{\prime} \neq i}\left(\frac{n-n_{i}}{n_{i}-1}\right) n_{i^{\prime}}
\end{aligned}
$$

Considerando (B.2) e o fato de que $2 \sum_{i<i^{\prime}} n_{i} n_{i^{\prime}}=\sum_{i \neq i^{\prime}} n_{i} n_{i^{\prime}}=\sum_{i=1}^{k} \sum_{i \neq i^{\prime}} n_{i} n_{i^{\prime}}$, podemos reescrever (B.1) como

$$
\begin{aligned}
M_{n}=\sum_{1 \leq r<s \leq n} \eta_{n r s}^{2} & =\frac{1}{2}\left\{\sum_{i=1}^{k} \sum_{i^{\prime} \neq i}\left(n-n_{i}\right) n_{i^{\prime}}+\sum_{i=1}^{k} \sum_{i^{\prime} \neq i}\left(\frac{n-n_{i}}{n_{i}-1}\right) n_{i^{\prime}}+\sum_{i \neq i^{\prime}} n_{i} n_{i^{\prime}}\right\} \\
& =\frac{1}{2}\left\{n \sum_{i=1}^{k} \sum_{i^{\prime} \neq i} n_{i^{\prime}}+n \sum_{i=1}^{k}\left(\frac{n-n_{i}}{n_{i}-1}\right)-\sum_{i=1}^{k} n_{i}\left(\frac{n-n_{i}}{n_{i}-1}\right)\right\} .
\end{aligned}
$$

Nobre, Juvêncio S. 
Finalmente, dado que $n \sum_{i=1}^{k} \sum_{i^{\prime} \neq i} n_{i^{\prime}}=n^{2}(k-1)$ e

$$
\begin{aligned}
\sum_{i=1}^{k} n_{i}\left(\frac{n-n_{i}}{n_{i}-1}\right) & =\sum_{i=1}^{k}\left(n-n_{i}\right)\left\{1+\frac{1}{n_{i}-1}\right\}=\sum_{i=1}^{k}\left(n-n_{i}\right)+\sum_{i=1}^{k}\left(\frac{n-n_{i}}{n_{i}-1}\right) \\
& =n(k-1)+\sum_{i=1}^{k}\left(\frac{n-n_{i}}{n_{i}-1}\right),
\end{aligned}
$$

temos

$$
\begin{aligned}
M_{n}=\sum_{1 \leq r<s \leq n} \eta_{n r s}^{2} & =\frac{1}{2}\left\{n^{2}(k-1)+n \sum_{i=1}^{k}\left(\frac{n-n_{i}}{n_{i}-1}\right)-n(k-1)-\sum_{i=1}^{k}\left(\frac{n-n_{i}}{n_{i}-1}\right)\right\} \\
& =\frac{1}{2}\left\{n(n-1)(k-1)+(n-1) \frac{n(k-1)}{n(k-1)} \sum_{i=1}^{k}\left(\frac{n-n_{i}}{n_{i}-1}\right)\right\} \\
& =\left(\begin{array}{l}
n \\
2
\end{array}\right)(k-1)\left\{1+\frac{1}{n} \sum_{i=1}^{k} \frac{n-n_{i}}{\left(n_{i}-1\right)(k-1)}\right\},
\end{aligned}
$$

provando (3.18).

\section{B.3 Teorema 3.1}

Primeiramente, notemos que $B_{n}=\left(\begin{array}{l}n \\ 2\end{array}\right)^{-1} \sum_{1 \leq r<s \leq n} \eta_{n r s} \psi\left(Y_{r}, Y_{s}\right)$ é uma soma de $\left(\begin{array}{l}n \\ 2\end{array}\right)$ termos, e que sob $\mathcal{H}_{0}: \sigma_{b}^{2}=0$, suas parcelas são não correlacionadas com $\mathbb{E}\left[\psi\left(Y_{r}, Y_{s}\right)\right]=0$ e $\operatorname{Var}\left[\psi\left(Y_{r}, Y_{s}\right)\right]=\sigma_{e}^{4}<\infty$. Desta forma, obtemos

$$
\begin{aligned}
\mathbb{E}\left[B_{n}\right] & =0 \\
\operatorname{Var}\left[B_{n}\right] & =\sigma_{e}^{4} M_{n}\left(\begin{array}{l}
n \\
2
\end{array}\right)^{-2} .
\end{aligned}
$$

Para obter a distribuição assintótica de $B_{n}$, podemos fazer uso de algumas versões do Teorema Limite Central (TLC) para variáveis dependentes; veja por exemplo, Dvoretzky (1972), Hall e Heyde (1980), Shiryayev (1983), Sen e Singer (1993), Lehmann (1998) e Chow e Teicher (2003). Aqui, decidimos por explorar a estrutura martingal da estatística de interesse $B_{n}$ para aplicar o TLC para martingais (Teorema A.1) como em Pinheiro et al. (2007). A demonstração a seguir é apresentada em Pinheiro et al. (2007) em um contexto mais geral, porém com $k$ fixo (na notação utilizada neste texto). Decidimos reproduzir os resultados e demonstrações de Pinheiro et al. (2007) com a pretensão de tornar a leitura deste texto mais 
auto-suficiente. Aqui, provamos que todas as condições de regularidade são válidas para o caso em que $k \rightarrow \infty$ e os $n_{i}$ 's são limitados (sendo também possível mostrar para o caso em que ambos $k \rightarrow \infty$ e $n_{i} \rightarrow \infty$, considerando condições de regularidade adicionais a respeito da seqüência $\left.\left\{n_{i}\right\}_{i=1, \ldots, k}\right)$.

Inicialmente, consideremos uma estatística da forma

$$
T_{n}=\sum_{1 \leq i<j \leq n} \eta_{n i j} \phi\left(X_{i}, X_{j}\right)
$$

com $\phi$ satisfazendo

$$
\begin{aligned}
& \phi_{1}\left(X_{1}\right)=\mathbb{E}\left[\phi\left(X_{1}, X_{2}\right) \mid X_{1}\right]=0 \text { q.c. (quase certamente), } \\
& \mathbb{E}\left[\phi\left(X_{1}, X_{2}\right) \phi\left(X_{1}, X_{3}\right)\right]=0 \\
& \mathbb{E}\left[\phi^{2}\left(X_{1}, X_{2}\right)\right]<\infty
\end{aligned}
$$

e com $X_{1}, \ldots, X_{n}$ representando uma seqüência de variáveis aleatórias independentes e identicamente distribuídas com função de distribuição $F$, com os coeficientes (pesos) $\eta_{n i j}$ sendo tais que

$$
\begin{aligned}
& \sum_{1 \leq i<j \leq n} \eta_{n i j}=0 \\
& \sum_{1 \leq i<j \leq n} \eta_{n i j}^{2}=M_{n}^{*}
\end{aligned}
$$

sendo $M_{n}^{*}$ uma função de $n$. Para os pesos definidos em (3.16), note que $M_{n}^{*}=M_{n}$ como definido em (3.18). Podemos considerar $T_{n}$ em (B.6) como função de vetores aleatórios iid, como em Pinheiro et al. (2007). A demonstração é análoga à apresentada a seguir; porém, por simplicidade de notação, consideraremos o caso univariado.

Definindo $Z_{n j}=\sum_{i=1}^{j-1} \eta_{n i j} \phi\left(X_{i}, X_{j}\right), \quad j=2, \ldots, n \mathrm{e}$

$$
T_{n u}=\sum_{l=2}^{u} Z_{n l}, \quad 2 \leq u \leq n,
$$

observamos que $T_{n n}=T_{n}$. Agora, consideremos a seqüência não-decrescente $(\mathrm{em} u)$ de $\sigma$-álgebras $\sigma_{n u}=\sigma\left(X_{i}, i \leq u\right)$ geradas pelos vetores aleatórios $\left(X_{1}, \ldots, X_{u}\right)^{\top}, 2 \leq u \leq n$.

Teorema B.1. (Pinheiro et al., 2007) Sob as condições (B.6)-(B.9), $\left\{T_{n u}, 2 \leq u \leq n\right\}$ é um processo martingal de média zero com respeito ao filtro $\left\{\sigma_{n u}, 2 \leq u \leq n\right\}$. 


\section{Prova}

Pelas definições de $\sigma_{n u}$ e (B.12) podemos concluir que $T_{n u}$ é $\sigma_{n u}$-mensurável. Além disso, dado (B.9), $T_{n u}$ é integrável. Agora, resta-nos mostrar que

$$
\mathbb{E}\left[T_{n} \mid \sigma_{n u}\right]=T_{n u} \text {, q.c. } 2 \leq u \leq n .
$$

Como $T_{n}=T_{n u}+\sum_{j=u+1}^{n} Z_{n j}$ para $u<n$, então é suficiente mostrar que $\forall u<j \leq n$

$$
\mathbb{E}\left[Z_{n j} \mid \sigma_{n u}\right]=0 \text { q.c. }
$$

Todavia, notemos que $\forall u<j \leq n$,

$$
\mathbb{E}\left[Z_{n j} \mid \sigma_{n u}\right]=\sum_{i=1}^{j-1} \eta_{n j} \mathbb{E}\left[\phi\left(X_{i}, X_{j}\right) \mid \sigma_{n u}\right]=0 \text { q.c., }
$$

pois se $i \leq u$ temos que $\mathbb{E}\left[\phi\left(X_{i}, X_{j}\right) \mid \sigma_{n u}\right]=\phi_{1}\left(X_{i}\right)=0$ q.c.; caso contrário, $\mathbb{E}\left[\phi\left(X_{i}, X_{j}\right) \mid \sigma_{n u}\right]$ $=\mathbb{E}\left[\phi\left(X_{i}, X_{j}\right)\right]=0$, demostrando assim o teorema.

Sejam $\tau^{2}=\mathbb{E}\left[\phi^{2}\left(X_{i}, X_{j}\right)\right]<\infty(i<j)$ e $D_{n}$ um estimador (fracamente) consistente de $\tau^{2}$. Por (B.8) e (B.9), temos

$$
\operatorname{Var}\left[T_{n}\right]=\mathbb{E}\left[T_{n}^{2}\right]=\tau^{2} M_{n}^{*}
$$

então, pelo Teorema de Slutsky

$$
\frac{D_{n} M_{n}^{*}}{\mathbb{E}\left[T_{n}^{2}\right]} \stackrel{\mathbb{P}}{\longrightarrow} 1
$$

A estatística $\left(\begin{array}{l}n \\ 2\end{array}\right) B_{n}$ tem a forma (B.6) em que $\phi(x, y)=(x-\mu)(y-\mu)$, com $\phi$ satisfazendo (B.7)-(B.9). Adicionalmente, temos $\tau^{2}=\mathbb{E}\left[\phi^{2}\left(X_{i}, X_{j}\right)\right]=\sigma_{e}^{4}<\infty$. Para o caso em questão, o lema abaixo, fornece um estimador consistente para $\tau^{2}$.

Lema B.1. Consideremos o modelo (3.1), a estatística $W_{n}=\sum_{i=1}^{k} \frac{n_{i}}{n} U_{i}=\sum_{i=1}^{k} w_{i} U_{i}$, com $U_{i}$ definida em (3.6). Sob a condições (3.3) $e$

$$
\max _{1 \leq i \leq k} \frac{n_{i}}{\sum_{i=1}^{k} n_{i}} \rightarrow 0
$$

então $W_{n} \stackrel{\mathbb{P}}{\longrightarrow} \sigma_{e}^{2}$.

Observemos que (B.14) é válido quando os $n_{i}$ 's são limitados (fixados) e $k \rightarrow \infty$. Além do 
mais, (B.14) também pode ser válido mesmo quando $n_{i} \rightarrow \infty$, por exemplo, em um estudo balanceado $\left(n_{i}=m, i=1, \ldots k\right)$.

\section{Prova}

Primeiramente, observemos que

$$
\mathbb{E}\left[W_{n}\right]=\sum_{i=1}^{k} \frac{n_{i}}{n} \mathbb{E}\left[U_{i}\right]=\sum_{i=1}^{k} \frac{n_{i}}{n} \sigma_{e}^{2}=\sigma_{e}^{2} .
$$

Por outro lado, $\forall \epsilon>0$, segue pela desigualdade de Chebychev que

$$
\begin{aligned}
\mathbb{P}\left[\left|W_{n}-\mathbb{E}\left[W_{n}\right]\right| \geq \epsilon\right] & \leq \frac{\operatorname{Var}\left[W_{n}\right]}{\epsilon^{2}} \\
& =(n \epsilon)^{-2} \sum_{i=1}^{k} n_{i}^{2} \operatorname{Var}\left[U_{i}\right]
\end{aligned}
$$

Todavia, como $n_{i} \geq 2$, então por (2.5) temos

$$
\begin{aligned}
n_{i}^{2} \operatorname{Var}\left[U_{i}\right] & =n_{i} \mu_{4 e}-\frac{n_{i}\left(n_{i}-3\right) \sigma_{e}^{4}}{n_{i}-1} \\
& \leq n_{i} \mu_{4 e}-\left(n_{i}-3\right) \sigma_{e}^{4} \\
& =n_{i}\left(\mu_{4 e}-\sigma_{e}^{4}\right)+3 \sigma_{e}^{4} \\
& \leq n_{i}\left(\mu_{4 e}+\sigma_{e}^{4}\right),
\end{aligned}
$$

o que implica

$$
\begin{aligned}
\mathbb{P}\left[\left|W_{n}-\mathbb{E}\left[W_{n}\right]\right| \geq \epsilon\right] & \leq \frac{\sum_{i=1}^{k} n_{i}^{2} \operatorname{Var}\left[U_{i}\right]}{n^{2} \epsilon^{2}} \\
& \leq \frac{\sum_{i=1}^{k} n_{i}\left(\mu_{4 e}+\sigma_{e}^{4}\right)}{n^{2} \epsilon^{2}} \\
& \leq \max _{1 \leq i \leq k} n_{i} \frac{\left(\mu_{4 e}+\sigma_{e}^{4}\right) k}{n^{2} \epsilon^{2}} \\
& <\left(\frac{\mu_{4 e}+\sigma_{e}^{4}}{\epsilon^{2}}\right) \max _{1 \leq i \leq k} \frac{n_{i}}{\sum_{i=1}^{k} n_{i}}
\end{aligned}
$$

dado que $k<n$. Portanto, sob a condição (B.14), $W_{n} \stackrel{\mathbb{P}}{\longrightarrow} \sigma_{e}^{2}$. Em algumas situações particulares (como no caso balanceado) pode-se mostrar este resultado (de convergência) sem a hipótese de existência de $\mathbb{E}\left[e_{i j}^{4}\right]$, usando a lei Fraca de Khintchine, por exemplo.

Antes de provar a validade do TLC para a estatística $\left(\begin{array}{l}n \\ 2\end{array}\right) B_{n}$, provaremos o seguinte lema: 
Lema B.2. Considere os pesos definidos em (3.16). Então, quando $k \rightarrow \infty$, temos

$$
\begin{aligned}
\sum_{1 \leq i \neq j<u \leq n} \eta_{n i u}^{2} \eta_{n j u}^{2} / M_{n}^{2} & \rightarrow 0, \\
\sum_{1 \leq i \neq j \leq n} \eta_{n i j}^{4} / M_{n}^{2} & \rightarrow 0 .
\end{aligned}
$$

\section{Prova}

Quando $k \rightarrow \infty$ e os $n_{i}$ 's são limitados, podemos concluir que $k=O(n)$. Então, por (3.18) obtemos que $M_{n}=\sum_{1 \leq r<s \leq n} \eta_{n r s}^{2}=O\left(n^{3}\right)$, implicando que $M_{n}^{2}=O\left(n^{6}\right)$. Por outro lado,

$$
\sum_{1 \leq i \neq j \leq n} \eta_{n i j}^{4}=\sum_{w=1}^{k}\left(\frac{n-n_{w}}{n_{w}-1}\right)^{4}\left(\begin{array}{c}
n_{w} \\
2
\end{array}\right)+\sum_{1 \leq w<w^{\prime} \leq k}(-1)^{4} n_{w} n_{w^{\prime}}=O\left(n^{5}\right),
$$

dado que a primeira soma tem $k$ termos, sendo cada um da mesma ordem de $n^{4}$, implicando que a primeira soma é $O\left(n^{5}\right)$; a segunda soma possui $\left(\begin{array}{l}k \\ 2\end{array}\right)$ termos, sendo todos os termos limitados, o que implica que a segunda soma é $O\left(n^{2}\right)$. Portanto,

$$
\sum_{1 \leq i \neq j \leq n} \eta_{n i j}^{4} / M_{n}^{2}=O\left(n^{-1}\right) \rightarrow 0
$$

quando $k \rightarrow \infty$, provando (B.16).

Como os $n_{i}$ 's são limitados

$$
\begin{aligned}
\sum_{1 \leq i \neq j<u \leq n} \eta_{n i u}^{2} \eta_{n j u}^{2} & \sim \sum_{w=1}^{k}\left(\frac{n-n_{w}}{n_{w}-1}\right)^{4}+\sum_{1 \leq w<w^{\prime} \leq k}\left(\frac{n-n_{w}}{n_{w}-1}\right)^{2}(-1)^{2}+\sum_{1 \leq w<w^{\prime}<w^{\prime \prime} \leq k}(-1)^{4} \\
& =O\left(n^{5}\right),
\end{aligned}
$$

dado que a primeira soma possui $k$ termos, sendo cada um $O\left(n^{4}\right)$; a segunda soma, possui $O\left(k^{2}\right)$ termos, sendo cada um da mesma ordem de $n^{2}$; por fim, a última parcela, possui $O\left(k^{3}\right)$ termos, sendo todos os termos limitados. Esses resultados implicam (B.15).

O teorema a seguir, constitui o teorema "chave" para a proposta.

Teorema B.2. (Pinheiro et al. 2007) Considere uma estatística da forma (B.6), com $\mathbb{E}\left[\phi^{4}\left(X_{1}, X_{2}\right)\right]<\infty$ e $D_{n}$ um estimador consistente de $\tau^{2}=\mathbb{E}\left[\phi^{2}\left(X_{1}, X_{2}\right)\right]$. Sob as hipóteses (B.7)-(B.9) e se os pesos $\eta_{\text {nij }}$ são tais que quando $n \rightarrow \infty$

$$
\begin{gathered}
\sum_{1 \leq i \neq j<k \leq n} \eta_{n i k}^{2} \eta_{n j k}^{2} /\left(M_{n}^{*}\right)^{2} \rightarrow 0 \\
\sum_{1 \leq i \neq j \leq n} \eta_{n i j}^{4} /\left(M_{n}^{*}\right)^{2} \rightarrow 0
\end{gathered}
$$

Nobre, Juvêncio S. 
então

$$
\left(M_{n}^{*} D_{n}\right)^{-1 / 2} T_{n} \stackrel{\mathcal{D}}{\longrightarrow} \mathcal{N}(0,1) .
$$

\section{Prova}

A demonstração é análoga à apresentada em Pinheiro et al. (2007) e será apresentada, como destacado anteriormente, com o objetivo de tornar a leitura auto-suficiente. Em particular, notemos que

$$
V_{n u}=\mathbb{E}\left[Z_{n u}^{2} \mid \sigma_{n(u-1)}\right], \quad 2 \leq u \leq n \text { e } V_{n}=\sum_{u=2}^{n} V_{n u},
$$

então segue, pelo Teorema B.1, que $\forall n \geq 2$,

$$
\begin{aligned}
\mathbb{E}\left[V_{n}\right] & =\sum_{u=2}^{n} \mathbb{E}\left[Z_{n u}^{2}\right]=\operatorname{Var}\left[\sum_{u=2}^{n} Z_{n u}\right] \\
& =\mathbb{E}\left[\left(\sum_{u=2}^{n} Z_{n u}\right)^{2}\right]=\mathbb{E}\left[T_{n}^{2}\right]
\end{aligned}
$$

Agora, observemos que

$$
\begin{aligned}
V_{n u}=\mathbb{E}\left[Z_{n u}^{2} \mid \sigma_{n(u-1)}\right] & =\mathbb{E}\left[\left(\sum_{i=1}^{u-1} \eta_{n i u} \phi\left(X_{i}, X_{u}\right)\right)^{2} \mid \sigma_{n(u-1)}\right] \\
& =\sum_{i<u} \eta_{n i u}^{2} \psi_{2}\left(X_{i}\right)+\sum_{1 \leq i \neq j<u} \eta_{n i u} \eta_{n j u} \psi_{3}\left(X_{i}, X_{j}\right),
\end{aligned}
$$

em que

$$
\begin{aligned}
\psi_{2}\left(X_{i}\right) & =\mathbb{E}\left[\phi^{2}\left(X_{i}, X_{u}\right) \mid X_{i}\right] \\
\psi_{3}\left(X_{i}, X_{j}\right) & =\mathbb{E}\left[\phi\left(X_{i}, X_{u}\right) \phi\left(X_{j}, X_{u}\right) \mid X_{i}, X_{j}\right](i \neq j),
\end{aligned}
$$

$\operatorname{com} \mathbb{E}\left[\psi_{2}\left(X_{i}\right)\right]=\tau^{2}$ e $\mathbb{E}\left[\psi_{3}\left(X_{i}, X_{j}\right)\right]=0(\forall i \neq j)$. Portanto,

$$
\mathbb{E}\left[V_{n u}\right]=\tau^{2} \sum_{i<u} \eta_{n i u}^{2} \forall u \leq n
$$

Notemos que

$$
\begin{aligned}
\frac{V_{n}}{\mathbb{E}\left[T_{n}^{2}\right]} & =\sum_{1 \leq i<j \leq n} \eta_{n i j}^{2} \psi_{2}\left(X_{i}\right) /\left(M_{n}^{*} \tau^{2}\right)+\sum_{1 \leq i \neq j<k \leq n} \eta_{n i k} \eta_{n j k} \psi_{3}\left(X_{i}, X_{j}\right) /\left(M_{n}^{*} \tau^{2}\right) \\
& =A_{1 n}+A_{2 n}
\end{aligned}
$$

Nobre, Juvêncio S. 
$\operatorname{com} A_{1 n}=\sum_{1 \leq i<j \leq n} \eta_{n i j}^{2} \psi_{2}\left(X_{i}\right) /\left(M_{n}^{*} \tau^{2}\right)$ e $A_{2 n}=\sum_{1 \leq i \neq j<k \leq n} \eta_{n i k} \eta_{n j k} \psi_{3}\left(X_{i}, X_{j}\right) /\left(M_{n}^{*} \tau^{2}\right)$. Por outro lado, $\mathbb{E}\left[A_{1 n}\right]=1$ e como, por hipótese, $\mathbb{E}\left[\phi^{4}\left(X_{1}, X_{2}\right)\right]<\infty$, temos

$$
\begin{gathered}
\operatorname{Var}\left[A_{1 n}\right]=\sum_{i<j} \eta_{n i j}^{4} \operatorname{Var}\left[\psi_{2}\left(X_{i}\right)\right] /\left(M_{n}^{*}\right)^{2} \tau^{4} \\
=\frac{\operatorname{Var}\left[\psi_{2}\left(X_{i}\right)\right]}{\tau^{4}} \sum_{1 \leq i \neq j \leq n} \eta_{n i j}^{4} /\left(M_{n}^{*}\right)^{2} \rightarrow 0
\end{gathered}
$$

implicando que $A_{1 n} \stackrel{\mathbb{P}}{\longrightarrow} 1$. Além disso, como $\mathbb{E}\left[A_{2 n}\right]=0$ e

$$
\operatorname{Var}\left[A_{2 n}\right]=\frac{\mathbb{E}\left[\psi_{3}^{2}\left(X_{1}, X_{2}\right)\right]}{\tau^{4}} \sum_{1 \leq i \neq j<k \leq n} \eta_{n i k}^{2} \eta_{n j k}^{2} /\left(M_{n}^{*}\right)^{2} \rightarrow 0
$$

pois $\mathbb{E}\left[\psi_{3}^{2}\left(X_{1}, X_{2}\right)\right] \leq \mathbb{E}\left[\phi^{2}\left(X_{i}, X_{u}\right) \phi^{2}\left(X_{j}, X_{u}\right)\right] \leq \mathbb{E}\left[\phi^{4}\left(X_{1}, X_{2}\right)\right]<\infty$, temos $A_{2 n} \stackrel{\mathbb{P}}{\longrightarrow} 0$. Portanto por (B.21) e pelo Teorema de Slutsky

$$
\frac{V_{n}}{\mathbb{E}\left[T_{n}^{2}\right]} \stackrel{\mathbb{P}}{\longrightarrow} 1
$$

Levando (B.22) em consideração, utilizaremos o TLC para arranjos martingais (veja Apêndice A.1) para provar (B.19), sendo para isso suficiente verificar a condição de Lindeberg, ou seja que

$$
\forall \epsilon>0, \quad \lim _{n \rightarrow \infty} \frac{1}{\mathbb{E}\left[T_{n}^{2}\right]} \sum_{u=2}^{n} \mathbb{E}\left[Z_{n u}^{2} \mathbb{1}\left\{\left|Z_{n u}\right|>\epsilon \sqrt{\mathbb{E}\left[T_{n}^{2}\right]}\right\}\right] \rightarrow 0
$$

Como a condição (B.23) é de difícil verificação, mostraremos que a condição de Liapunov (mais restritiva)

$$
\lim _{n \rightarrow \infty} \frac{1}{\mathbb{E}\left[T_{n}^{2}\right]^{2}} \sum_{u=2}^{n} \mathbb{E}\left[Z_{n u}^{4}\right] \rightarrow 0
$$

é válida, implicando a validade da condição de Lindeberg (B.23).

Por (B.8), (B.9) e (3.18), obtemos que $\operatorname{Var}\left[T_{n}\right]=\mathbb{E}\left[T_{n}^{2}\right]=\tau^{2} M_{n}=O\left(n^{3}\right)$, implicando que $\left(\mathbb{E}\left[T_{n}^{2}\right]\right)^{2}=O\left(n^{6}\right)$. Por outro lado, pelo teorema Multinomial e pela linearidade da integral 
de Lebesgue-Stieltjes temos que, para $2 \leq u \leq n$ :

$$
\begin{aligned}
\mathbb{E}\left[Z_{n u}^{4}\right] & =\mathbb{E}\left[\left(\sum_{i=1}^{u-1} \eta_{\text {niu }} \phi\left(X_{i}, X_{u}\right)\right)^{4}\right] \\
& =\sum_{i=1}^{u-1} \eta_{\text {niu }}^{4} \mathbb{E}\left[\phi^{4}\left(X_{i}, X_{u}\right)\right]+3 \sum_{1 \leq i \neq j<u} \eta_{n i u}^{2} \eta_{n j u}^{2} \mathbb{E}\left[\phi^{2}\left(X_{i}, X_{u}\right) \phi^{2}\left(X_{j}, X_{u}\right)\right] \\
& +4 \sum_{1 \leq i \neq j<u} \eta_{n i u}^{3} \eta_{n j u} \mathbb{E}\left[\phi^{3}\left(X_{i}, X_{u}\right) \phi\left(X_{j}, X_{u}\right)\right] \\
& +6 \sum_{1 \leq i \neq j \neq t<u} \eta_{n i u}^{2} \eta_{n j u} \eta_{n t u} \mathbb{E}\left[\phi^{2}\left(X_{i}, X_{u}\right) \phi\left(X_{j}, X_{u}\right) \phi\left(X_{t}, X_{u}\right)\right] \\
& +\sum_{1 \leq i \neq j \neq t \neq w<u} \eta_{n i u} \eta_{n j u} \eta_{n t u} \eta_{n w u} \mathbb{E}\left[\phi\left(X_{i}, X_{u}\right) \phi\left(X_{j}, X_{u}\right) \phi\left(X_{t}, X_{u}\right) \phi\left(X_{w}, X_{u}\right)\right] .
\end{aligned}
$$

Usando o fato de que $X_{1}, \ldots, X_{n}$ são iid e (B.7), temos que $\forall i \neq j<u$ :

$$
\begin{aligned}
\mathbb{E}\left[\phi^{3}\left(X_{i}, X_{u}\right) \phi\left(X_{j}, X_{u}\right)\right] & =\mathbb{E}\left[\mathbb{E}\left[\phi^{3}\left(X_{i}, X_{u}\right) \phi\left(X_{j}, X_{u}\right) \mid X_{u}\right]\right] \\
& =\mathbb{E}\left[\mathbb{E}\left[\phi^{3}\left(X_{i}, X_{u}\right) \mid X_{u}\right] \mathbb{E}\left[\phi\left(X_{j}, X_{u}\right) \mid X_{u}\right]\right]=0 .
\end{aligned}
$$

De forma análoga, podemos mostrar que

$$
\begin{aligned}
& \mathbb{E}\left[\phi^{2}\left(X_{i}, X_{u}\right) \phi\left(X_{j}, X_{u}\right) \phi\left(X_{t}, X_{u}\right)\right]=0 \forall i \neq j \neq t<u \\
& \mathbb{E}\left[\phi\left(X_{i}, X_{u}\right) \phi\left(X_{j}, X_{u}\right) \phi\left(X_{t}, X_{u}\right) \phi\left(X_{w}, X_{u}\right)\right]=0 \forall i \neq j \neq t \neq w<u,
\end{aligned}
$$

e conseqüentemente concluir

$$
\begin{aligned}
\mathbb{E}\left[Z_{n u}^{4}\right] & =\sum_{i=1}^{u-1} \eta_{n i u}^{4} \mathbb{E}\left[\phi^{4}\left(X_{i}, X_{u}\right)\right]+3 \sum_{1 \leq i \neq j<u} \eta_{n i u}^{2} \eta_{n j u}^{2} \mathbb{E}\left[\phi^{2}\left(X_{i}, X_{u}\right) \phi^{2}\left(X_{j}, X_{u}\right)\right] \\
& =\tau_{1} \sum_{i=1}^{u-1} \eta_{n i u}^{4}+\tau_{2} \sum_{1 \leq i \neq j<u} \eta_{n i u}^{2} \eta_{n j u}^{2},
\end{aligned}
$$

$\operatorname{com} \tau_{1}=\mathbb{E}\left[\phi^{4}\left(X_{i}, X_{u}\right)\right]<\infty$ e $\tau_{2}=3 \mathbb{E}\left[\phi^{2}\left(X_{i}, X_{u}\right) \phi^{2}\left(X_{j}, X_{u}\right)\right]<\infty, 1 \leq i \neq j<u$. Notemos que $\tau_{1}$ e $\tau_{2}$ independem de índices (constantes) dada a suposição de que $X_{1}, \ldots, X_{n}$ são iid.

Logo,

$$
\sum_{u=2}^{n} \mathbb{E}\left[Z_{n u}^{4}\right]=\tau_{1} \sum_{1 \leq i<k \leq n} \eta_{n i k}^{4}+\tau_{2} \sum_{1 \leq i \neq j<k \leq n} \eta_{n i k}^{2} \eta_{n j k}^{2}=O\left(n^{5}\right)
$$

como demonstrado anteriormente. Portanto, a condição de Liapunov (B.24) é válida, e conseqüentemente a de Lindeberg (B.23) também será, desde que

$$
\frac{1}{\mathbb{E}\left[T_{n}^{2}\right]^{2}} \sum_{u=2}^{n} \mathbb{E}\left[Z_{n u}^{4}\right]=O\left(n^{-1}\right)
$$

Nobre, Juvêncio S. 
Dessa forma, pelo Teorema A.1, podemos concluir que

$$
\left(M_{n}^{*} \tau^{2}\right)^{-1 / 2} T_{n} \stackrel{\mathcal{D}}{\longrightarrow} \mathcal{N}(0,1)
$$

Usando o fato de que $D_{n}$ é um estimador fracamente consistente para $\tau^{2}$ e fazendo uso do Teorema de Slutsky, provamos (B.19), o que conclui a demonstração.

Como a estatística $\left(\begin{array}{l}n \\ 2\end{array}\right) B_{n}$ tem a forma (B.6) $\operatorname{com} \phi(x, y)=(x-\mu)(y-\mu)$, em que $\phi$ satisfazendo (B.7)-(B.9) e $\mathbb{E}\left[\phi^{4}\left(X_{1}, X_{2}\right)\right]<\infty$ então o Teorema 3.1 pode ser provado a partir dos Lemas (B.1) e (B.2).

\section{B.4 Teorema 3.2}

O objetivo desta seção é obter a distribuição da estatística de teste $J_{n}$ definida em (3.21) para a seguinte seqüência de hipóteses locais de Pitman

$$
\mathcal{H}_{1 n}: \sigma_{b}^{2}=\delta^{2} / a_{n}
$$

quando $n \rightarrow \infty$, com $\left\{a_{n}\right\}_{n \geq 1}$ representando uma seqüência de números reais positivos, tal que $\lim _{n \rightarrow \infty} a_{n}=\infty$ e $\delta$ uma constante. A taxa de crescimento da seqüência $\left\{a_{n}\right\}_{n \geq 1}$ será discutida adiante.

Primeiramente observemos que

$$
U_{i}=\left(\begin{array}{c}
n_{i} \\
2
\end{array}\right)^{-1} \sum_{1 \leq j<j^{\prime} \leq n_{i}} \frac{\left(Y_{i j}-Y_{i j^{\prime}}\right)^{2}}{2}=\left(\begin{array}{c}
n_{i} \\
2
\end{array}\right)^{-1} \sum_{1 \leq j<j^{\prime} \leq n_{i}} \frac{\left(e_{i j}-e_{i j^{\prime}}\right)^{2}}{2}
$$

e

$$
W_{n}=\sum_{i=1}^{k} \frac{n_{i}}{n} U_{i}
$$

são funções apenas do vetor de erros condicionais e. Conseqüentemente, suas respectivas distribuições são invariantes sob $\mathcal{H}_{0}$ e $\mathcal{H}_{1 n}$. Por outro lado, temos

$$
\begin{aligned}
B_{n} & =\sum_{1 \leq i<i^{\prime} \leq k} \frac{n_{i} n_{i^{\prime}}}{n(n-1)}\left\{2 U_{i i^{\prime}}-U_{i}-U_{i^{\prime}}\right\} \\
& =\sum_{1 \leq i<i^{\prime} \leq k} \frac{n_{i} n_{i^{\prime}}}{n(n-1)}\left\{\frac{2}{n_{i} n_{i^{\prime}}} \sum_{j=1}^{n_{i}} \sum_{j^{\prime}=1}^{n_{i^{\prime}}} \frac{\left(Y_{i j}-Y_{i^{\prime} j^{\prime}}\right)^{2}}{2}-U_{i}-U_{i^{\prime}}\right\} \\
& =B_{n}^{0}+C_{n},
\end{aligned}
$$

Nobre, Juvêncio S. 
em que

$$
\begin{aligned}
B_{n}^{0} & =\sum_{1 \leq i<i^{\prime} \leq k} \frac{n_{i} n_{i^{\prime}}}{n(n-1)}\left\{\frac{2}{n_{i} n_{i^{\prime}}} \sum_{j=1}^{n_{i}} \sum_{j^{\prime}=1}^{n_{i^{\prime}}} \frac{\left(e_{i j}-e_{i^{\prime} j^{\prime}}\right)^{2}}{2}-U_{i}-U_{i^{\prime}}\right\}, \\
C_{n} & =\sum_{1 \leq i<i^{\prime} \leq k} \frac{n_{i} n_{i^{\prime}}}{n(n-1)}\left\{\left(b_{i}-b_{i^{\prime}}\right)^{2}+\frac{2\left(b_{i}-b_{i^{\prime}}\right)}{n_{i} n_{i^{\prime}}} \sum_{j=1}^{n_{i}} \sum_{j^{\prime}=1}^{n_{i^{\prime}}}\left(e_{i j}-e_{i^{\prime} j^{\prime}}\right)\right\},
\end{aligned}
$$

além disso, sob $\mathcal{H}_{0}, B_{n} \stackrel{\text { q.c. }}{=} B_{n}^{0}$, dado que $C_{n}=0$ com probabilidade 1. Portanto,

$$
J_{n}^{*}=\frac{\left(\begin{array}{c}
n \\
2
\end{array}\right) B_{n}}{\sigma_{e}^{2} M_{n}^{1 / 2}}=\frac{\left(\begin{array}{c}
n \\
2
\end{array}\right) B_{n}^{0}}{\sigma_{e}^{2} M_{n}^{1 / 2}}+\frac{\left(\begin{array}{c}
n \\
2
\end{array}\right) C_{n}}{\sigma_{e}^{2} M_{n}^{1 / 2}}=J_{n 0}+Q_{n},
$$

em que $Q_{n}=\left(\begin{array}{c}n \\ 2\end{array}\right) C_{n} /\left(\sigma_{e}^{2} M_{n}^{1 / 2}\right)$ e $J_{n 0}=\left(\begin{array}{c}n \\ 2\end{array}\right) B_{n}^{0} /\left(\sigma_{e}^{2} M_{n}^{1 / 2}\right) \stackrel{\mathcal{D}}{\longrightarrow} \mathcal{N}(0,1)$ quando $k \rightarrow \infty$ (ver Seção B.3). Consideremos a decomposição

$$
C_{n}=C_{n 1}+C_{n 2}
$$

em que

$$
\begin{aligned}
C_{n 1} & =\frac{1}{n(n-1)} \sum_{1 \leq i \leq i^{\prime} \leq k} n_{i} n_{i^{\prime}}\left(b_{i}-b_{i^{\prime}}\right)^{2}, \\
C_{n 2} & =\frac{2}{n(n-1)} \sum_{1 \leq i \leq i^{\prime} \leq k}\left(b_{i}-b_{i^{\prime}}\right) \sum_{j=1}^{n_{i}} \sum_{j^{\prime}=1}^{n_{i^{\prime}}}\left(e_{i j}-e_{i j^{\prime}}\right) .
\end{aligned}
$$

Como $\mathbb{E}\left[C_{n 2}\right]=0$, temos

$$
\begin{aligned}
\mathbb{E}\left[C_{n}\right] & =\mathbb{E}\left[C_{n 1}\right]=\frac{1}{n(n-1)} \sum_{1 \leq i \leq i^{\prime} \leq k} n_{i} n_{i^{\prime}} \mathbb{E}\left[\left(b_{i}-b_{i^{\prime}}\right)^{2}\right] \\
& =\frac{1}{n(n-1)} \sum_{1 \leq i \leq i^{\prime} \leq k} n_{i} n_{i^{\prime}} 2 \sigma_{b}^{2} \\
& =\sigma_{b}^{2}\left(\frac{n^{2}-\sum_{i=1}^{k} n_{i}^{2}}{n(n-1)}\right),
\end{aligned}
$$

de tal forma que sob $\mathcal{H}_{1 n}: \sigma_{b}^{2}=\delta^{2} / a_{n}$

$$
\mathbb{E}\left[C_{n}\right]=\frac{\delta^{2}\left(n^{2}-\sum_{i=1}^{k} n_{i}^{2}\right)}{a_{n} n(n-1)}=O\left(a_{n}^{-1}\right) .
$$

O resultado a seguir nos permite avaliar o comportamento assintótico de $Q_{n}$ para a seqüência $a_{n}=\sqrt{n}=O\left(\left(\begin{array}{c}n \\ 2\end{array}\right) / M_{n}^{1 / 2}\right)$ sob a suposição da existência do quarto momento da distribuição dos efeitos aleatórios. 
Lema B.3. Consideremos o modelo (3.1) sob a seqüência $\mathcal{H}_{1 n}: \sigma_{b}^{2}=\delta^{2} / \sqrt{n}$ e $\lim _{n \rightarrow \infty} M_{n} / n^{3}=$ $\lambda$. Sob as condições (3.2)-(3.4) e se $\mathbb{E}\left[b_{i}^{4}\right]<\infty$, temos

$$
\frac{\left(\begin{array}{l}
n \\
2
\end{array}\right) C_{n}}{\sqrt{M_{n}}} \stackrel{\mathbb{P}}{\longrightarrow} \frac{\delta^{2}}{2 \sqrt{\lambda}} .
$$

\section{Prova}

Dado que $\mathbb{E}\left[b_{i}^{4}\right]<\infty$, então pela contigüidade da seqüência de hipóteses locais $\mathcal{H}_{1 n}$ : $\sigma_{b}^{2}=\delta^{2} / \sqrt{n}$, temos que $\mathbb{E}\left[b_{i}^{4}\right]=o(1)$ sob $\mathcal{H}_{1 n}$. Definindo $b^{i j}=\left(b_{i}-b_{j}\right)^{2}$, e lembrando que $b_{1}, \ldots, b_{k}$ são iid e que os $n_{i}$ 's são limitados, temos

$$
\begin{aligned}
\operatorname{Var}\left[C_{n 1}\right] & =\frac{1}{n^{2}(n-1)^{2}}\left\{\sum_{1 \leq i<i^{\prime} \leq k} n_{i}^{2} n_{i^{\prime}}^{2} \operatorname{Var}\left[b^{i i^{\prime}}\right]+\sum_{\substack{1 \leq i<t \leq k \\
1 \leq i<w \leq k \\
t \neq w}} n_{i}^{2} n_{t} n_{w} \operatorname{Cov}\left[b^{i t}, b^{i w}\right]\right\} \\
& \leq \frac{\max \left\{\operatorname{Var}\left[b^{12}\right], \operatorname{Cov}\left[b^{12}, b^{13}\right]\right\}}{n^{2}(n-1)^{2}}\left\{\sum_{\substack{1 \leq i<i^{\prime} \leq k \\
n_{i}}} n_{i}^{2} n_{i^{\prime}}^{2} \sum_{\substack{1 \leq i<t \leq k \\
1 \leq i<w \leq k \\
t \neq w}} n_{i}^{2} n_{t} n_{w}\right\} \\
& =o(1) O\left(n^{-1}\right)=o\left(n^{-1}\right),
\end{aligned}
$$

dado que $\operatorname{Var}\left[b^{12}\right]$ e $\operatorname{Cov}\left[b^{12}, b^{13}\right]$ convergem para zero quando $k \rightarrow \infty$ (na mesma ordem que $\left.\mathbb{E}\left[b_{i}^{4}\right]\right)$, isto é, ambos são $o(1)$. De forma similar, obtemos $\operatorname{Var}\left[C_{n 2}\right]=o\left(n^{-1}\right)$. Agora, como $\left(\begin{array}{l}n \\ 2\end{array}\right) / \sqrt{M_{n}}=O(\sqrt{n})$, obtemos

$$
\lim _{n \rightarrow \infty} \frac{\left(\begin{array}{c}
n \\
2
\end{array}\right) \mathbb{E}\left[C_{n 1}\right]}{\sqrt{M_{n}}}=\lim _{n \rightarrow \infty} \frac{\delta^{2}\left(n^{2}-\sum_{i=1}^{k} n_{i}^{2}\right)}{2 \sqrt{n} \sqrt{M_{n}}}=\frac{\delta^{2}}{2 \sqrt{\lambda}},
$$

e como $O(n) \operatorname{Var}\left[C_{n 1}\right]=o(1)$, concluímos que

$$
\frac{\left(\begin{array}{l}
n \\
2
\end{array}\right) C_{n 1}}{\sqrt{M_{n}}} \stackrel{\mathbb{P}}{\longrightarrow} \frac{\delta^{2}}{2 \sqrt{\lambda}},
$$

dado que se $\mathbb{E}\left[X_{n}\right] \rightarrow c$ e $\operatorname{Var}\left[X_{n}\right] \rightarrow 0$, então $X_{n} \stackrel{\mathbb{P}}{\longrightarrow} c$. Analogamente, como $\mathbb{E}\left[C_{n 2}\right]=0$ e $\operatorname{Var}\left[C_{n 2}\right]=o\left(n^{-1}\right)$, obtemos

$$
\frac{\left(\begin{array}{l}
n \\
2
\end{array}\right) C_{n 2}}{\sqrt{M_{n}}} \stackrel{\mathbb{P}}{\longrightarrow} 0 .
$$

Portanto,

$$
\frac{\left(\begin{array}{l}
n \\
2
\end{array}\right) C_{n}}{\sqrt{M_{n}}}=\frac{\left(\begin{array}{l}
n \\
2
\end{array}\right)\left(C_{n 1}+C_{n 2}\right)}{\sqrt{M_{n}}} \stackrel{\mathbb{P}}{\longrightarrow} \frac{\delta^{2}}{2 \sqrt{\lambda}} .
$$

Nobre, Juvêncio S. 
Considerando (B.26) e o Lema B.3, lembrando que $W_{n} \stackrel{\mathbb{P}}{\longrightarrow} \sigma_{e}^{2}$, e utilizando o teorema de Slutsky, obtemos

$$
J_{n}=\frac{\left(\begin{array}{l}
n \\
2
\end{array}\right) B_{n}}{W_{n} \sqrt{M_{n}}}=\frac{\left(\begin{array}{c}
n \\
2
\end{array}\right) B_{n}^{0}}{\sigma_{e}^{2} \sqrt{M_{n}}} \frac{\sigma_{e}^{2}}{W_{n}}+\frac{\left(\begin{array}{l}
n \\
2
\end{array}\right) C_{n}}{\sigma_{e}^{2} \sqrt{M_{n}}} \frac{\sigma_{e}^{2}}{W_{n}} \stackrel{\mathcal{D}}{\longrightarrow} \mathcal{N}\left(\frac{\delta^{2}}{2 \sigma_{e}^{2} \sqrt{\lambda}}, 1\right),
$$

implicando que, sob $\mathcal{H}_{1 n}: \sigma_{b}^{2}=\delta^{2} / \sqrt{n}$, a distribuição da estatística $J_{n}$ é afetada apenas no parâmetro de localização, que é transladado à direita, com a seguinte magnitude da translação

$$
\frac{\delta^{2}}{2 \sigma_{e}^{2} \sqrt{\lambda}}
$$

\section{B.5 MINQUE de $\sigma_{e}^{2}$}

Re-escrevamos o modelo (1.2) $\operatorname{com} \boldsymbol{\Sigma}=\sigma_{e}^{2} \mathbf{I}_{n}$ na seguinte forma

$$
\mathbf{Y}=\mathbf{X} \boldsymbol{\beta}+\mathbf{Z b}+\mathbf{e}=\mathbf{W} \boldsymbol{\beta}^{*}+\mathbf{e}
$$

$\operatorname{com} \mathbf{W}=(\mathbf{X}, \mathbf{Z})$ e $\boldsymbol{\beta}^{*}=(\boldsymbol{\beta}, \mathbf{b})^{\top}$. Em geral a matriz $\mathbf{W}$ não é de posto completo, dado que comumente $\mathcal{C}(\mathbf{Z}) \subset \mathcal{C}(\mathbf{X}), \operatorname{com} \mathcal{C}(\mathbf{M})$ denotando o espaço gerado pelas colunas da matriz $\mathbf{M}$ (espaço coluna). Denotando $\mathbf{M}^{-}$uma inversa generalizada da matriz $\mathbf{M}$ e $p^{*}=\operatorname{posto}(\mathbf{W})$, consideremos o MINQUE de $\sigma_{e}^{2}$ :

$$
S_{n}=\frac{\mathbf{Y}^{\top}\left(\mathbf{I}_{n}-\mathbf{W}\left(\mathbf{W}^{\top} \mathbf{W}\right)^{-} \mathbf{W}^{\top}\right) \mathbf{Y}}{n-p^{*}} .
$$

De agora em diante, adicionaremos um índice $n$ a todos os vetores e matrizes em questão, para evidenciar a dependência com relação ao tamanho da amostra. O seguinte resultado estabelece a invariância e a consistência do estimador $S_{n}$ sob algumas condições de regularidade.

Resultado 1. Seja $S_{n}$ como definido em (B.28), $\mathbf{W}_{n}=\left(\mathbf{X}_{n}, \mathbf{Z}_{n}\right)$, com $\mathbf{X}_{n}$ e $\mathbf{Z}_{n}$ definidos em (B.27) com $\mathbf{w}_{n k}^{\top}$ denotando a k-ésima linha de $\mathbf{W}_{n}$. Então, $S_{n}$ é um estimador não viesado para $\sigma_{e}^{2}$ e é invariante com relação à escolha da inversa generalizada. Supondo adicionalmente que

$$
\lim _{n \rightarrow \infty} \max _{1 \leq k \leq n} \mathbf{w}_{n k}^{\top}\left(\mathbf{W}_{n}^{\top} \mathbf{W}_{n}\right)^{-} \mathbf{w}_{n k} \rightarrow 0
$$

temos que

$$
S_{n} \stackrel{\mathbb{P}}{\longrightarrow} \sigma_{e}^{2}
$$

Nobre, Juvêncio S. 


\section{Prova}

Seja A uma matriz qualquer, representemos o espaço gerado pelas colunas (linhas) de $\mathbf{A}$ por $\mathcal{C}(\mathbf{A})(\mathcal{R}(\mathbf{A}))$. Dado que $\mathcal{C}\left(\mathbf{W}_{n}^{\top}\right)=\mathcal{C}\left(\mathbf{W}_{n}^{\top} \mathbf{W}_{n}\right)$ e $\mathcal{R}\left(\mathbf{W}_{n}\right)=\mathcal{R}\left(\mathbf{W}_{n}^{\top} \mathbf{W}_{n}\right)$ (Harville, 1997, p.75), então o produto $\mathbf{W}_{n}\left(\mathbf{W}_{n}^{\top} \mathbf{W}_{n}\right)^{-} \mathbf{W}_{n}^{\top}$ é invariante com relação a escolha da inversa generalizada $\left(\mathbf{W}_{n}^{\top} \mathbf{W}_{n}\right)^{-}$; veja a demonstração em Harville (1997, p.119), por exemplo . Sob a validade do modelo (1.2), $\mathbf{W}_{n}^{\top}\left(\mathbf{I}_{n}-\mathbf{W}_{n}\left(\mathbf{W}_{n}^{\top} \mathbf{W}_{n}\right)^{-} \mathbf{W}_{n}^{\top}\right) \mathbf{W}_{n}=\mathbf{0}$, de forma que podemos reescrever o estimador (B.28) como

$$
\begin{aligned}
S_{n} & =\frac{\left(\mathbf{W}_{n} \boldsymbol{\beta}^{*}+\mathbf{e}_{n}\right)^{\top}\left(\mathbf{I}_{n}-\mathbf{W}_{n}\left(\mathbf{W}_{n}^{\top} \mathbf{W}_{n}\right)^{-} \mathbf{W}_{n}^{\top}\right)\left(\mathbf{W}_{n} \boldsymbol{\beta}^{*}+\mathbf{e}_{n}\right)}{n-p^{*}} \\
& =\frac{\mathbf{e}_{n}^{\top}\left(\mathbf{I}_{n}-\mathbf{W}_{n}\left(\mathbf{W}_{n}^{\top} \mathbf{W}_{n}\right)^{-} \mathbf{W}_{n}^{\top}\right) \mathbf{e}_{n}}{n-p^{*}}
\end{aligned}
$$

Dado que $\mathbb{E}\left[\mathbf{e}_{n}\right]=\mathbf{0}$ e $\boldsymbol{\Sigma}_{\mathbf{e}_{n}}=\sigma_{e}^{2} \mathbf{I}_{n}$, então $\mathbb{E}\left[\mathbf{e}_{n}^{\top} \mathbf{A} \mathbf{e}_{n}\right]=\operatorname{tr}\left(\mathbf{A} \boldsymbol{\Sigma}_{\mathbf{e}_{n}}\right)=\sigma_{e}^{2} \operatorname{tr}(\mathbf{A})$, implicando

$$
\begin{aligned}
\mathbb{E}\left[\mathbf{e}_{n}^{\top}\left(\mathbf{W}_{n}\left(\mathbf{W}_{n}^{\top} \mathbf{W}_{n}\right)^{-} \mathbf{W}_{n}^{\top}\right) \mathbf{e}_{n}\right] & =\sigma_{e}^{2} \operatorname{tr}\left(\mathbf{W}_{n}\left(\mathbf{W}_{n}^{\top} \mathbf{W}_{n}\right)^{-} \mathbf{W}_{n}^{\top}\right) \\
& =\sigma_{e}^{2} \operatorname{posto}\left(\mathbf{W}_{n}\left(\mathbf{W}_{n}^{\top} \mathbf{W}_{n}\right)^{-} \mathbf{W}_{n}^{\top}\right) \\
& =\sigma_{e}^{2} \operatorname{posto}\left(\mathbf{W}_{n}\right)=\sigma_{e}^{2} p^{*}
\end{aligned}
$$

Por outro lado, como $\mathbb{E}\left[\mathbf{e}_{n}^{\top} \mathbf{e}_{n}\right]=n \sigma_{e}^{2}$, (B.28) é um estimador não viesado de $\sigma_{e}^{2}$. Para a prova da consistência, veja por exemplo, Brown (1976) e Demidenko (2004).

O método para obtenção de estimadores quadráticos não viesados de norma mínima foi proposto por Rao (1970, 1971a). Para obter esses estimadores basta assumir a existência do quarto momento da fonte de variação em questão (no nosso caso, do erro condicional). Detalhes a respeito deste método de estimação para componentes de variância, propriedades assintóticas desta classe de estimadores e métodos relacionados podem ser encontrados em Rao (1970, 1971a, 1971b, 1972), Brown (1976), Rao e Kleffe (1988), McCulloch e Searle (2001), Demidenko (2004) e em Jiang (2007), por exemplo.

Um outro estimador consistente de $\sigma_{e}^{2}$ no modelo (1.2) obtido sob normalidade apenas do vetor de erros condicionais e sob o modelo é o EMV ou EMVR; esse estimador é consistente mesmo em situações em que a distribuição dos efeitos aleatórios não seja normal, mas tenha o terceiro momento finito. Para detalhes, veja por exemplo, Verbeke e Lesaffre (1996, 1997). Tais estimadores estão disponíveis na maioria dos "software" padrão (SAS, S, R, por exemplo). Desta forma, sua utilização depende apenas da suposição de normalidade do erro condicional, e para este fim, sugerimos que leitor consulte Nobre (2004, Cap.2) ou Nobre e Singer (2007), que versam sobre análise de resíduos em modelos lineares mistos. 


\section{B.6 Teorema 3.3}

Levando em consideração (3.25), notemos primeiramente que

$$
\begin{aligned}
T_{n \beta} & =\sum_{1 \leq r<s \leq n} \eta_{n r s}\left(Y_{s}-\mathbf{x}_{n s}^{\top} \boldsymbol{\beta}\right)\left(Y_{r}-\mathbf{x}_{n r}^{\top} \boldsymbol{\beta}\right) \\
& =\sum_{1 \leq r<s \leq n} \eta_{n r s}\left(Y_{s}-\mathbf{x}_{n s}^{\top} \widehat{\boldsymbol{\beta}}_{n}\right)\left(Y_{r}-\mathbf{x}_{n r}^{\top} \widehat{\boldsymbol{\beta}}_{n}\right)+\sum_{1 \leq r<s \leq n} \eta_{n r s} \mathbf{x}_{n s}^{\top}\left(\widehat{\boldsymbol{\beta}}_{n}-\boldsymbol{\beta}\right)\left(Y_{r}-\mathbf{x}_{n r}^{\top} \boldsymbol{\beta}\right) \\
& +\sum_{1 \leq r<s \leq n} \eta_{n r s}\left(Y_{s}-\mathbf{x}_{n s}^{\top} \widehat{\boldsymbol{\beta}}_{n}\right) \mathbf{x}_{n r}^{\top}\left(\widehat{\boldsymbol{\beta}}_{n}-\boldsymbol{\beta}\right) \\
& =T_{n \hat{\boldsymbol{\beta}}}+T_{n 1}+T_{n 2},
\end{aligned}
$$

com $T_{n 1}$ e $T_{n 2}$ dados, respectivamente, por

$$
\begin{aligned}
T_{n 1} & =\sum_{1 \leq r<s \leq n} \eta_{n r s} \mathbf{x}_{n s}^{\top}\left(\widehat{\boldsymbol{\beta}}_{n}-\boldsymbol{\beta}\right)\left(Y_{r}-\mathbf{x}_{n r}^{\top} \boldsymbol{\beta}\right) \\
T_{n 2} & =\sum_{1 \leq r<s \leq n} \eta_{n r s}\left(Y_{s}-\mathbf{x}_{n s}^{\top} \widehat{\boldsymbol{\beta}}_{n}\right) \mathbf{x}_{n r}^{\top}\left(\widehat{\boldsymbol{\beta}}_{n}-\boldsymbol{\beta}\right) .
\end{aligned}
$$

Antes de provar o teorema, considere o seguinte lema que fornece informação importante para estabelecermos o comportamento assintótico de $T_{n \hat{\beta}}$.

Lema B.4. Consideremos o modelo (3.23), $T_{n 1}$ e $T_{n 2}$ dados, respectivamente, em (B.31) e (B.32) e seja $\mathbf{x}_{n s}^{\top}$ a s-ésima linha da matriz $\mathbf{X}_{n}$. Sob as suposições (3.2)-(3.4) e se existe uma constante $\gamma<\infty$ tal que $e^{1}$

$$
\sup _{1 \leq s \leq n}\left\|\mathbf{x}_{n s}\right\| \leq \gamma
$$

então, para $i=1,2$, temos

$$
\frac{T_{n i}}{\sqrt{M_{n}}} \stackrel{\mathbb{P}}{\longrightarrow} 0
$$

\section{Prova}

Sob a validade do modelo (3.23) e sob $\mathcal{H}_{0}$, temos

$$
\begin{aligned}
\widehat{\boldsymbol{\beta}}_{n} & =\left(\mathbf{X}_{n}^{\top} \mathbf{X}_{n}\right)^{-1} \mathbf{X}_{n}^{\top} \mathbf{Y}_{n}=\left(\mathbf{X}_{n}^{\top} \mathbf{X}_{n}\right)^{-1} \mathbf{X}_{n}^{\top}\left(\mathbf{X}_{n} \boldsymbol{\beta}+\mathbf{e}_{n}\right) \\
& =\boldsymbol{\beta}+\left(\mathbf{X}_{n}^{\top} \mathbf{X}_{n}\right)^{-1} \mathbf{X}_{n}^{\top} \mathbf{e}_{n} .
\end{aligned}
$$

Pelo TLC de Hájek-Šidak temos que $\left(\widehat{\boldsymbol{\beta}}_{n}-\boldsymbol{\beta}\right)=O_{p}\left(n^{-1 / 2}\right)$, e por (B.34) obtemos

$$
\widehat{\boldsymbol{\beta}}_{n}-\boldsymbol{\beta}=\left(\mathbf{X}_{n}^{\top} \mathbf{X}_{n}\right)^{-1} \mathbf{X}_{n}^{\top} \mathbf{e}_{n}=O_{p}\left(n^{-1 / 2}\right)=o_{p}(1) .
$$

${ }^{1}$ Esta é uma condição de regularidade usual para provar propriedades assintóticas em modelos lineares mistos; veja, por exemplo, em Demidenko (2004) e Jiang (2007). 
Denotando o $r$-ésimo elemento do vetor $\mathbf{e}_{n}$ por $e_{n r}$, notemos que

$$
\begin{aligned}
\mathbf{x}_{n s}^{\top}\left(\widehat{\boldsymbol{\beta}}_{n}-\boldsymbol{\beta}\right)\left(Y_{r}-\mathbf{x}_{r}^{\top} \boldsymbol{\beta}\right) & =\mathbf{x}_{n s}^{\top}\left(\mathbf{X}_{n}^{\top} \mathbf{X}_{n}\right)^{-1} \mathbf{X}_{n}^{\top} \mathbf{e}_{n}\left(Y_{r}-\mathbf{x}_{r}^{\top} \boldsymbol{\beta}\right) \\
& =\mathbf{x}_{n s}^{\top}\left(\mathbf{X}_{n}^{\top} \mathbf{X}_{n}\right)^{-1} \mathbf{X}_{n}^{\top} \mathbf{e}_{n} e_{n r} .
\end{aligned}
$$

Portanto,

$$
\begin{aligned}
T_{n 1} & =\sum_{1 \leq r<s \leq n} \eta_{n r s} \mathbf{x}_{n s}^{\top}\left(\widehat{\boldsymbol{\beta}}_{n}-\boldsymbol{\beta}\right)\left(Y_{r}-\mathbf{x}_{n r}^{\top} \boldsymbol{\beta}\right)=\sum_{1 \leq r<s \leq n} \eta_{n r s} \mathbf{x}_{n s}^{\top}\left(\mathbf{X}_{n}^{\top} \mathbf{X}_{n}\right)^{-1} \mathbf{X}_{n}^{\top} \mathbf{e}_{n} e_{n r} \\
& =\mathbf{e}_{n}^{\top} \mathbf{X}_{n}\left(\mathbf{X}_{n}^{\top} \mathbf{X}_{n}\right)^{-1} \sum_{1 \leq r<s \leq n} \eta_{n r s} \mathbf{x}_{n s} e_{n r}=\mathbf{e}_{n}^{\top} \mathbf{X}_{n}\left(\mathbf{X}_{n}^{\top} \mathbf{X}_{n}\right)^{-1} \mathbf{T}_{n 1},
\end{aligned}
$$

em que $\mathbf{T}_{n 1}=\sum_{1 \leq r<s \leq n} \eta_{n r s} \mathbf{x}_{n s} e_{n r}$ é um vetor aleatório $p$-dimensional com vetor de médias e matriz de covariâncias dados, respectivamente, por

$$
\begin{aligned}
\mathbb{E}\left[\mathbf{T}_{n 1}\right] & =\mathbf{0} \\
\Sigma_{\mathbf{T}_{n 1}} & =\sigma_{e}^{2}\left\{\sum_{1 \leq r<s \leq n} \eta_{n r s}^{2} \mathbf{x}_{n s} \mathbf{x}_{n s}^{\top}+\sum_{1 \leq r<s \neq s_{1} \leq n} \eta_{n r s} \eta_{n r s_{1}} \mathbf{x}_{n s} \mathbf{x}_{n s_{1}}^{\top}\right\} .
\end{aligned}
$$

Por (B.33), os elementos das matrizes $\mathbf{x}_{n s} \mathbf{x}_{n s_{1}}^{\top}, 1 \leq s, s_{1} \leq n$ são limitados; conseqüentemente $\Sigma_{\mathbf{T}_{n 1}}=O\left(M_{n}\right)^{2}$. Portanto, podemos mostrar que

$$
\mathbf{T}_{n 1}=\sum_{1 \leq r<s \leq n} \eta_{n r s} \mathbf{x}_{n s} e_{n r}=O_{p}\left(\sqrt{M_{n}}\right)
$$

o que nos levar a concluir

$$
\frac{T_{n 1}}{\sqrt{M_{n}}}=\mathbf{e}_{n}^{\top} \mathbf{X}_{n}\left(\mathbf{X}_{n}^{\top} \mathbf{X}_{n}\right)^{-1} \frac{\mathbf{T}_{n 1}}{\sqrt{M_{n}}}=O_{p}\left(n^{-1 / 2}\right) O_{p}(1)=O_{p}\left(n^{-1 / 2}\right)=o_{p}(1)
$$

isto é,

$$
\frac{T_{n 1}}{\sqrt{M_{n}}} \stackrel{\mathbb{P}}{\longrightarrow} 0
$$

Por outro lado, notemos que

$$
\begin{aligned}
T_{n 2} & =\sum_{1 \leq r<s \leq n} \eta_{n r s}\left(Y_{s}-\mathbf{x}_{n s}^{\top} \widehat{\boldsymbol{\beta}}_{n}\right) \mathbf{x}_{n r}^{\top}\left(\widehat{\boldsymbol{\beta}}_{n}-\boldsymbol{\beta}\right)=\left(\widehat{\boldsymbol{\beta}}_{n}-\boldsymbol{\beta}\right)^{\top} \sum_{1 \leq r<s \leq n} \eta_{n r s}\left(Y_{s}-\mathbf{x}_{n s}^{\top} \widehat{\boldsymbol{\beta}}_{n}\right) \mathbf{x}_{n r} \\
& =\mathbf{e}_{n}^{\top} \mathbf{X}_{n}\left(\mathbf{X}_{n}^{\top} \mathbf{X}_{n}\right)^{-1} \sum_{1 \leq r<s \leq n} \eta_{n r s}\left(Y_{s}-\mathbf{x}_{n s}^{\top} \widehat{\boldsymbol{\beta}}_{n}\right) \mathbf{x}_{n r} .
\end{aligned}
$$

Considerando a matriz de projeção $\mathbf{H}_{n}=\mathbf{X}_{n}\left(\mathbf{X}_{n}^{\top} \mathbf{X}_{n}\right)^{-1} \mathbf{X}_{n}^{\top}$, o vetor de resíduos marginais $\widehat{\mathbf{e}}_{n}=\mathbf{Y}_{n}-\mathbf{X}_{n} \widehat{\boldsymbol{\beta}}_{n}=\left(\mathbf{I}_{n}-\mathbf{H}_{n}\right) \mathbf{Y}_{n}=\left(\mathbf{I}_{n}-\mathbf{H}_{n}\right) \mathbf{e}_{n}$ e denotando o elemento da $i$-ésima linha e $j$-ésima coluna de $\mathbf{H}_{n}$ por $h_{n i j}$, a primeira condição generalizada de Noether (item i), p. 31), pode ser reescrita como

${ }^{2}$ Dizemos que $\mathbf{A}=O\left(b_{n}\right)$ se $\|\mathbf{A}\|=O\left(b_{n}\right)$, ou de forma equivalente se vec $(\mathbf{A})=O\left(b_{n}\right)$, com $\|\mathbf{A}\|=$ $\|\operatorname{vec}(\mathbf{A})\|$ denotando a norma de Frobenius da matriz A.

Nobre, Juvêncio S. 
i') $\max _{1 \leq k \leq n} h_{n k k} \rightarrow 0$, quando $n \rightarrow \infty$.

Além disso, como $\mathbf{H}_{n}$ é uma matriz simétrica e idempotente, podemos mostrar que $0 \leq$ $h_{n i i} \leq 1$ e $\left|h_{n i j}\right|=\left|h_{n j i}\right| \leq \min \left\{\sqrt{h_{n j j}\left(1-h_{n j j}\right)}, \sqrt{h_{n i i}\left(1-h_{n i i}\right)}\right\}$, para $1 \leq i, j \leq n$. Portanto a condição i') é equivalente a

i") $\max _{\substack{1 \leq i \leq n \\ 1 \leq j \leq n}} h_{n i j} \rightarrow 0$, quando $n \rightarrow \infty$.

Denotando o $s$-ésimo elemento do vetor $\widehat{\mathbf{e}}_{n}$ por $\hat{e}_{n s}$, que é uma combinação linear do vetor de erros condicionais, segue que

$$
\begin{aligned}
T_{n 2} & =\mathbf{e}_{n}^{\top} \mathbf{X}_{n}\left(\mathbf{X}_{n}^{\top} \mathbf{X}_{n}\right)^{-1} \sum_{1 \leq r<s \leq n} \eta_{n r s}\left(Y_{s}-\mathbf{x}_{n s}^{\top} \widehat{\boldsymbol{\beta}}_{n}\right) \mathbf{x}_{n r} \\
& =\mathbf{e}_{n}^{\top} \mathbf{X}_{n}\left(\mathbf{X}_{n}^{\top} \mathbf{X}_{n}\right)^{-1} \sum_{1 \leq r<s \leq n} \eta_{n r s} \mathbf{x}_{n r} \hat{e}_{n s}=\mathbf{e}_{n}^{\top} \mathbf{X}_{n}\left(\mathbf{X}_{n}^{\top} \mathbf{X}_{n}\right)^{-1} \mathbf{T}_{n 2}
\end{aligned}
$$

em que $\mathbf{T}_{n 2}=\sum_{1 \leq r<s \leq n} \eta_{n r s} \mathbf{x}_{n s} \hat{e}_{n s}$. Dado que $\operatorname{Var}\left[\hat{e}_{n s}\right]=\sigma_{e}^{2}\left(1-h_{n s s}\right)$ e $\operatorname{Cov}\left[\hat{e}_{n s}, \hat{e}_{n t}\right]=$ $-\sigma_{e}^{2} h_{n s t}$, então de forma análoga ao caso anterior, podemos mostrar que

$$
\mathbf{T}_{n 2}=O_{p}\left(\sqrt{M_{n}}\right)
$$

implicando

$$
\frac{T_{n 2}}{\sqrt{M_{n}}}=\mathbf{e}_{n}^{\top} \mathbf{X}_{n}\left(\mathbf{X}_{n}^{\top} \mathbf{X}_{n}\right)^{-1} \frac{\mathbf{T}_{n 2}}{\sqrt{M_{n}}}=O_{p}\left(n^{-1 / 2}\right) O_{p}(1)=O_{p}\left(n^{-1 / 2}\right)=o_{p}(1) .
$$

Por conseguinte,

$$
\begin{aligned}
\frac{T_{n \hat{\beta}}}{\sigma_{e}^{2} \sqrt{M_{n}}} & =\frac{T_{n \beta}}{\sigma_{e}^{2} \sqrt{M_{n}}}-\frac{\left(T_{n 1}+T_{n 2}\right)}{\sigma_{e}^{2} \sqrt{M_{n}}} \\
& =J_{n \beta}-\frac{\left(T_{n 1}+T_{n 2}\right)}{\sigma_{e}^{2} \sqrt{M_{n}}} .
\end{aligned}
$$

Agora levando em consideração o resultado do Lema B.4, o fato de que $J_{n \beta} \stackrel{\mathcal{D}}{\longrightarrow} \mathcal{N}(0,1)$ e que $S_{n} \stackrel{\mathbb{P}}{\longrightarrow} \sigma_{e}^{2}$, quando $k \rightarrow \infty$, e fazendo uso do Teorema de Slutsky, concluímos que

$$
J_{n \widehat{\beta}} \stackrel{\mathcal{D}}{\longrightarrow} \mathcal{N}(0,1)
$$

quando $k \rightarrow \infty$, provando o teorema. 


\section{B.7 Teorema 3.4}

Pelo Teorema 3.3 e considerando $J_{n \hat{\beta}}$ definida em (3.28), sob $\mathcal{H}_{0}: \sigma_{b}^{2}=0$, temos

$$
J_{n \hat{\beta}} \stackrel{\mathcal{D}}{\longrightarrow} \mathcal{N}(0,1)
$$

quando $k \rightarrow \infty$. Sob a seqüência $\mathcal{H}_{1 n}: \sigma_{b}^{2}=\delta^{2} / \sqrt{n}$, podemos reescrever a estatística $T_{n \hat{\beta}}$ definida em (3.27) como

$$
\begin{aligned}
T_{n \hat{\beta}} & =\sum_{1 \leq r<s \leq n} \eta_{n r s}\left(\mathbf{x}_{n r}^{\top} \boldsymbol{\beta}+b_{u(r)}+e_{n r}-\mathbf{x}_{n r}^{\top} \widehat{\boldsymbol{\beta}}_{n}\right)\left(\mathbf{x}_{n s}^{\top} \boldsymbol{\beta}+b_{u(s)}+e_{n s}-\mathbf{x}_{n r}^{\top} \widehat{\boldsymbol{\beta}}_{n}\right) \\
& =\sum_{1 \leq r<s \leq n} \eta_{n r s}\left(\mathbf{x}_{n r}^{\top} \boldsymbol{\beta}+e_{n r}-\mathbf{x}_{n r}^{\top} \widehat{\boldsymbol{\beta}}_{n}\right)\left(\mathbf{x}_{n s}^{\top} \boldsymbol{\beta}+e_{n s}-\mathbf{x}_{n r}^{\top} \widehat{\boldsymbol{\beta}}_{n}\right) \\
& +\sum_{1 \leq r<s \leq n} \eta_{n r s} b_{u(r)}\left(\mathbf{x}_{n s}^{\top}\left(\boldsymbol{\beta}-\widehat{\boldsymbol{\beta}}_{n}\right)+e_{n s}\right) \\
& +\sum_{1 \leq r<s \leq n} \eta_{n r s} b_{u(s)}\left(\mathbf{x}_{n r}^{\top}\left(\boldsymbol{\beta}-\widehat{\boldsymbol{\beta}}_{n}\right)+e_{n r}\right) \\
& +\sum_{1 \leq r<s \leq n} \eta_{n r s} b_{u(s)} b_{u(r)} \\
& =T_{n \hat{\beta}}^{0}+A_{n 1}+A_{n 2}+A_{n 3},
\end{aligned}
$$

com

$$
\begin{aligned}
T_{n \hat{\beta}}^{0} & =\sum_{1 \leq r<s \leq n} \eta_{n r s}\left(\mathbf{x}_{n r}^{\top} \boldsymbol{\beta}+e_{n r}-\mathbf{x}_{n r}^{\top} \widehat{\boldsymbol{\beta}}_{n}\right)\left(\mathbf{x}_{n s}^{\top} \boldsymbol{\beta}+e_{n s}-\mathbf{x}_{n r}^{\top} \widehat{\boldsymbol{\beta}}_{n}\right), \\
A_{n 1} & =\sum_{1 \leq r<s \leq n} \eta_{n r s} b_{u(r)}\left(\mathbf{x}_{n s}^{\top}\left(\boldsymbol{\beta}-\widehat{\boldsymbol{\beta}}_{n}\right)+e_{n s}\right), \\
A_{n 2} & =\sum_{1 \leq r<s \leq n} \eta_{n r s} b_{u(s)}\left(\mathbf{x}_{n r}^{\top}\left(\boldsymbol{\beta}-\widehat{\boldsymbol{\beta}}_{n}\right)+e_{n r}\right) \mathrm{e} \\
A_{n 3} & =\sum_{1 \leq r<s \leq n} \eta_{n r s} b_{u(s)} b_{u(r)} .
\end{aligned}
$$

Sob a hipótese nula $\mathcal{H}_{0}: \sigma_{b}^{2}=0$, temos que $T_{n \hat{\beta}}=T_{n \hat{\beta}}^{0}$ quase certamente. A função $u(s)$ refere-se ao índice da unidade amostral correspondente a $s$-ésima observação ordenada lexicograficamente. Para ilustrar, consideremos a situação em que $k=3 \operatorname{com} n_{1}=2, n_{2}=3$ e $n_{3}=4$. Assim $n=\sum_{i=1}^{3} n_{i}=9$, de tal forma que

$$
\begin{aligned}
& u(1)=u(2)=1, \\
& u(3)=u(4)=u(5)=2, \quad \mathrm{e} \\
& u(6)=u(7)=u(8)=u(9)=3 .
\end{aligned}
$$

Nobre, Juvêncio S. 
As variáveis aleatórias $b_{u(r)}$ e $b_{u(s)}$ são independentes se e somente se $Y_{r}$ e $Y_{s}$ não corresponderem a observações de uma mesma unidade amostral, de tal forma que $\operatorname{Cov}\left[b_{u(r)}, b_{u(s)}\right]=$ $\mathbb{E}\left[b_{u(r)} b_{u(s)}\right]=\left(\delta^{2} / \sqrt{n}\right) \mathbb{1}\{u(r)=u(s)\}$.

Podemos decompor $A_{n 1}$ como

$$
\begin{aligned}
A_{n 1} & =\sum_{1 \leq r<s \leq n} \eta_{n r s} b_{u(r)}\left(\mathbf{x}_{n s}^{\top}\left(\boldsymbol{\beta}-\widehat{\boldsymbol{\beta}}_{n}\right)+e_{n s}\right) \\
& =\left(\boldsymbol{\beta}-\widehat{\boldsymbol{\beta}}_{n}\right)^{\top} \sum_{1 \leq r<s \leq n} \eta_{n r s} b_{u(r)} \mathbf{x}_{n s}^{\top}+\sum_{1 \leq r<s \leq n} \eta_{n r s} b_{u(r)} e_{n s},
\end{aligned}
$$

e concluir que $\mathbb{E}\left[A_{n 1}\right]=0$. Analogamente, podemos mostrar que $\mathbb{E}\left[A_{n 2}\right]=0$. Por outro lado,

$$
\begin{aligned}
\mathbb{E}\left[A_{n 3}\right] & =\sum_{1 \leq r<s \leq n} \eta_{n r s} \mathbb{E}\left[b_{u(r)} b_{u(s)}\right]=\sum_{1 \leq r<s \leq n} \eta_{n r s}\left(\delta^{2} / \sqrt{n}\right) \mathbb{1}\{u(r)=u(s)\} \\
& =\frac{\delta^{2}}{\sqrt{n}} \sum_{i=1}^{k}\left(\frac{n-n_{i}}{n_{i}-1}\right)\left(\begin{array}{c}
n_{i} \\
2
\end{array}\right) \\
& =\frac{\delta^{2}}{2 \sqrt{n}} \sum_{i=1}^{k}\left(n-n_{i}\right) n_{i} \\
& =\frac{\delta^{2}}{2 \sqrt{n}}\left(n^{2}-\sum_{i=1}^{k} n_{i}^{2}\right) .
\end{aligned}
$$

Lembrando que $J_{n \hat{\beta}}=T_{n \hat{\beta}} /\left(S_{n} \sqrt{M_{n}}\right)$, então por (B.36) temos

$$
J_{n \hat{\beta}}=J_{n \hat{\beta}}^{0}+A_{n 1}^{*}+A_{n 2}^{*}+A_{n 3}^{*}
$$

em que $J_{n \hat{\beta}}^{0}=T_{n \hat{\beta}}^{0} /\left(S_{n} \sqrt{M_{n}}\right) \stackrel{\mathcal{D}}{\longrightarrow} \mathcal{N}(0,1)$ quando $k \rightarrow \infty$ (pelo Teorema 3.1) e $A_{n i}^{*}=$ $A_{n i} /\left(S_{n} \sqrt{M_{n}}\right)$ para $i=1,2,3$. Observemos que

$$
A_{n 1}^{*}=\frac{1}{S_{n}}\left\{\frac{\left(\boldsymbol{\beta}-\widehat{\boldsymbol{\beta}}_{n}\right)^{\top}}{\sqrt{M_{n}}} \sum_{1 \leq r<s \leq n} \eta_{n r s} b_{u(r)} \mathbf{x}_{n s}+\frac{1}{\sqrt{M_{n}}} \sum_{1 \leq r<s \leq n} \eta_{n r s} b_{u(r)} e_{n s}\right\} ;
$$

além disso, $\mathbf{T}_{n 1}^{*}=\sum_{1 \leq r<s \leq n} \eta_{n r s} b_{u(r)} \mathbf{x}_{n s}$ é um vetor aleatório $p$-dimensional, com vetor de médias e matriz de covariâncias dados, respectivamente, por

$$
\begin{aligned}
\mathbb{E}\left[\mathbf{T}_{n 1}^{*}\right] & =\mathbf{0} \\
\Sigma_{\mathbf{T}_{n 1}^{*}} & =\frac{\delta^{2}}{\sqrt{n}}\left\{\sum_{i=1}^{k}\left(\frac{n-n_{i}}{n_{i}-1}\right)^{2} \sum_{1 \leq j<j^{\prime} \leq n_{i}} \mathbf{x}_{n j} \mathbf{x}_{n j^{\prime}}^{\top}\right\}=O\left(n^{3 / 2}\right)=o\left(M_{n}\right) .
\end{aligned}
$$

Nobre, Juvêncio S. 
Considerando a suposição do Lema B.4, segue que $\mathbf{T}_{n 1}^{*} / \sqrt{M_{n}}=o_{p}(1)$. Analogamente, é possível mostrar que

$$
\frac{1}{\sqrt{M_{n}}} \sum_{1 \leq r<s \leq n} \eta_{n r s} b_{u(r)} e_{s}=o_{p}(1)
$$

de forma que

$$
\begin{aligned}
A_{n 1}^{*} & =\frac{1}{S_{n}}\left\{\frac{\left(\boldsymbol{\beta}-\widehat{\boldsymbol{\beta}}_{n}\right)^{\top}}{\sqrt{M_{n}}} \sum_{1 \leq r<s \leq n} \eta_{n r s} b_{u(r)} \mathbf{x}_{n s}+\frac{1}{\sqrt{M_{n}}} \sum_{1 \leq r<s \leq n} \eta_{n r s} b_{u(r)} e_{n s}\right\} \\
& =\frac{1}{S_{n}}\left\{O_{p}\left(n^{-1 / 2}\right) o_{p}(1)+o_{p}(1)\right\}=o_{p}(1)
\end{aligned}
$$

desde que $S_{n} \stackrel{\mathbb{P}}{\longrightarrow} \sigma_{e}^{2}$. Similarmente, podemos provar que

$$
A_{n 2}^{*}=\frac{1}{S_{n} \sqrt{M_{n}}} \sum_{1 \leq r<s \leq n} \eta_{n r s} b_{u(s)}\left(\mathbf{x}_{n r}^{\top}\left(\boldsymbol{\beta}-\widehat{\boldsymbol{\beta}}_{n}\right)+e_{n r}\right)=o_{p}(1) .
$$

Para finalizar a demonstração, necessitamos provar que $A_{n 3}^{*} \stackrel{\mathbb{P}}{\longrightarrow} \delta^{2} /\left(\sigma_{e}^{2} \sqrt{\lambda}\right)$. Com esta finalidade, notemos que

$$
\begin{aligned}
A_{n 3} & =\sum_{1 \leq r<s \leq n} \eta_{n r s} b_{u(s)} b_{u(r)}=\sum_{i=1}^{k}\left(\frac{n-n_{i}}{n_{i}-2}\right)\left(\begin{array}{c}
n_{i} \\
2
\end{array}\right) b_{i}^{2}-\sum_{i<i^{\prime}} n_{i} n_{i^{\prime}} b_{i} b_{i^{\prime}} \\
& =\frac{1}{2}\left\{\sum_{i=1}^{k}\left(n-n_{i}\right) n_{i} b_{i}^{2}-\sum_{i \neq i^{\prime}} n_{i} n_{i^{\prime}} b_{i} b_{i^{\prime}}\right\} \\
& =A_{n 31}-A_{n 32}
\end{aligned}
$$

em que $A_{n 31}=\frac{1}{2} \sum_{i=1}^{k}\left(n-n_{i}\right) n_{i} b_{i}^{2}$ e $A_{n 32}=\frac{1}{2} \sum_{i \neq i^{\prime}} n_{i} n_{i^{\prime}} b_{i} b_{i^{\prime}}$, de forma que $\mathbb{E}\left[A_{n 3}\right]=\mathbb{E}\left[A_{n 31}\right]$ e $\mathbb{E}\left[A_{n 32}\right]=0$. Denotando $\lim _{n \rightarrow \infty} M_{n} / n^{3}=\lambda$, então

$$
\lim _{n \rightarrow \infty} \frac{\mathbb{E}\left[A_{n 3}\right]}{\sqrt{M_{n}}}=\lim _{n \rightarrow \infty} \frac{\mathbb{E}\left[A_{n 31}\right]}{\sqrt{M_{n}}}=\lim _{n \rightarrow \infty} \frac{\delta^{2}\left(n^{2}-\sum_{i=1}^{k} n_{i}^{2}\right)}{2 \sqrt{n M_{n}}}=\frac{\delta^{2}}{2 \sqrt{\lambda}} .
$$

Lembrando que $b_{1}, \ldots, b_{k}$ são iid e que os $n_{i}$ 's são limitados,

$$
\operatorname{Var}\left[A_{n 31}\right]=\frac{1}{4} \sum_{i=1}^{k}\left(n-n_{i}\right)^{2} n_{i}^{2} \operatorname{Var}\left[b_{i}^{2}\right]=\frac{1}{4} \operatorname{Var}\left[b_{1}^{2}\right] \sum_{i=1}^{k}\left(n-n_{i}\right)^{2} n_{i}^{2}=\operatorname{Var}\left[b_{1}^{2}\right] O\left(M_{n}\right)
$$

dado que sob a seqüência $\mathcal{H}_{1 n}$ temos $\operatorname{Var}\left[b_{1}^{2}\right]=o(1)$, uma vez que $\mathbb{E}\left[b_{1}^{4}\right]=o(1)$ (pela contigüidade da seqüência $\left.\mathcal{H}_{1 n}\right)$, implicando que $\operatorname{Var}\left[A_{n 31}\right]=o\left(M_{n}\right)$. Analogamente, podemos mostrar que $\operatorname{Var}\left[A_{n 32}\right]=o\left(M_{n}\right)$, de forma que podemos concluir

$$
\frac{A_{n 31}}{\sqrt{M_{n}}} \stackrel{\mathbb{P}}{\longrightarrow} \lim _{n \rightarrow \infty} \frac{\mathbb{E}\left[A_{n 31}\right]}{\sqrt{M_{n}}}=\frac{\delta^{2}}{2 \sqrt{\lambda}}
$$

Nobre, Juvêncio S. 
e

$$
\frac{A_{n 32}}{\sqrt{M_{n}}} \stackrel{\mathbb{P}}{\longrightarrow} 0
$$

Logo,

$$
\frac{A_{n 3}}{\sqrt{M_{n}}}=\frac{A_{n 31}}{\sqrt{M_{n}}}+\frac{A_{n 32}}{\sqrt{M_{n}}} \stackrel{\mathbb{P}}{\longrightarrow} \frac{\delta^{2}}{2 \sqrt{\lambda}} .
$$

Agora, usando o fato de que $S_{n} \stackrel{\mathbb{P}}{\longrightarrow} \sigma_{e}^{2}$, os resultados (B.37), (B.38), (B.39) e o Teorema de Slutsky, concluímos

$$
J_{n \hat{\beta}}=J_{n \hat{\beta}}^{0}+A_{n 1}^{*}+A_{n 2}^{*}+A_{n 3}^{*} \stackrel{\mathcal{D}}{\longrightarrow} \mathcal{N}\left(\frac{\delta^{2}}{2 \sigma_{e}^{2} \sqrt{\lambda}}, 1\right),
$$

e então sob $\mathcal{H}_{1 n}: \sigma_{b}^{2}=\delta^{2} / \sqrt{n}$, a distribuição da estatística $J_{n \hat{\beta}}$ sob $\mathcal{H}_{1 n}$ é afetada apenas no parâmetro de localização, no sentido de a média da distribuição normal ser deslocada à direita, com translação de magnitude

$$
\frac{\delta^{2}}{2 \sigma_{e}^{2} \sqrt{\lambda}}
$$

que é exatamente a mesma distribuição obtida no caso em que a média da variável resposta não é modelada por meio de variáveis explicativas.

\section{B.8 Demonstração das condições de regularidade (3.45)}

Como $\mathbf{V}_{n}=\bigoplus_{i=1}^{k} \mathbf{V}_{i}$, com $\mathbf{V}_{i}=\sigma_{e}^{2} \mathbf{I}_{n_{i}}+\sigma_{0}^{2} \mathbf{J}_{n_{i}}$, então $\mathbf{V}_{n}^{-1}=\bigoplus_{i=1}^{k} \mathbf{V}_{i}^{-1}$. Podemos mostrar que a inversa da matriz $\mathbf{V}_{i}$ (veja por exemplo, Press, 2005, p. 23) é dada por

$$
\mathbf{V}_{i}^{-1}=\frac{1}{\sigma_{e}^{2}} \mathbf{I}_{n_{i}}-\frac{\sigma_{0}^{2}}{\sigma_{e}^{2}\left(\sigma_{e}^{2}+\sigma_{0}^{2} n_{i}\right)} \mathbf{J}_{n_{i}}
$$

e conseqüentemente,

$$
\widehat{\mathbf{V}}_{i}^{-1}=\frac{1}{S_{n}} \mathbf{I}_{n_{i}}-\frac{S_{0}}{S_{n}\left(S_{n}+S_{0} n_{i}\right)} \mathbf{J}_{n_{i}}
$$

Dado que para $n_{i}$ fixado, $f_{1}\left(S_{n}\right)=\left(S_{n}\right)^{-1}$ e $f_{2}\left(S_{n}, S_{0}\right)=S_{0} /\left(S_{n}\left(S_{n}+S_{0} n_{i}\right)\right)$ são funções (q.c.) contínuas de $\left(S_{n}, S_{0}\right)$ então $f_{1}\left(S_{n}\right) \stackrel{\mathbb{P}}{\longrightarrow} f_{1}\left(\sigma_{e}^{2}\right)$ e $f_{2}\left(S_{n}, S_{0}\right) \stackrel{\mathbb{P}}{\longrightarrow} f_{2}\left(\sigma_{e}^{2}, \sigma_{0}^{2}\right)$, implicando que $\widehat{\mathbf{V}}_{i}^{-1} \stackrel{\mathbb{P}}{\longrightarrow} \mathbf{V}_{i}^{-1}$.

Além disso, notemos que

$$
\mathbf{V}_{n} \widehat{\mathbf{V}}_{n}^{-1}-\mathbf{I}_{n}=\bigoplus_{i=1}^{k} \mathbf{V}_{i} \widehat{\mathbf{V}}_{i}^{-1}-\mathbf{I}_{n}=\bigoplus_{i=1}^{k}\left(\mathbf{V}_{i} \widehat{\mathbf{V}}_{i}^{-1}-\mathbf{I}_{n_{i}}\right)
$$

Nobre, Juvêncio S. 
é uma matriz em blocos diagonal, e seu polinômio característico, é dado por (para detalhes, veja Harville, 1997, por exemplo)

$$
p_{n}(\lambda)=\prod_{i=1}^{k} p_{i}(\lambda)
$$

com $p_{i}(\lambda)$ representando o polinômio característico da matriz $\mathbf{V}_{i} \widehat{\mathbf{V}}_{i}^{-1}-\mathbf{I}_{n_{i}}$. Por conseguinte, uma condição necessária e suficiente para que os autovalores de $\mathbf{V}_{n} \widehat{\mathbf{V}}_{n}^{-1}-\mathbf{I}_{n}$ convirjam em probabilidade para zero é que os autovalores de $\mathbf{V}_{i} \widehat{\mathbf{V}}_{i}^{-1}-\mathbf{I}_{n_{i}}$ convirjam em probabilidade para zero. Pela definição de $\mathbf{V}_{i}$ e por (B.40), temos

$$
\begin{aligned}
\mathbf{V}_{i} \widehat{\mathbf{V}}_{i}^{-1} & =\left(\sigma_{e}^{2} \mathbf{I}_{n_{i}}+\sigma_{0}^{2} \mathbf{J}_{n_{i}}\right)\left(\frac{1}{S_{n}} \mathbf{I}_{n_{i}}-\frac{S_{0}}{S_{n}\left(S_{n}+S_{0} n_{i}\right)} \mathbf{J}_{n_{i}}\right) \\
& =\frac{\sigma_{e}^{2}}{S_{n}} \mathbf{I}_{n_{i}}-\left\{\frac{S_{0}\left(\sigma_{e}^{2}+n_{i} \sigma_{0}^{2}\right)}{S_{n}\left(S_{n}+n_{i} S_{0}\right)}-\frac{\sigma_{0}^{2}}{S_{n}}\right\} \mathbf{J}_{n_{i}},
\end{aligned}
$$

$\log 0$

$$
\mathbf{V}_{i} \widehat{\mathbf{V}}_{i}^{-1}-\mathbf{I}_{n_{i}}=\left\{\frac{\sigma_{e}^{2}}{S_{n}}-1\right\} \mathbf{I}_{n_{i}}-\left\{\frac{S_{0}\left(\sigma_{e}^{2}+n_{i} \sigma_{0}^{2}\right)}{S_{n}\left(S_{n}+n_{i} S_{0}\right)}-\frac{\sigma_{0}^{2}}{S_{n}}\right\} \mathbf{J}_{n_{i}}
$$

que é uma matriz uniforme. Portanto, os autovalores de $\mathbf{V}_{i} \widehat{\mathbf{V}}_{i}^{-1}-\mathbf{I}_{n_{i}}$ (veja por exemplo, Press, 2005, p. 29), são

$$
\lambda_{1_{n}}=a_{n}^{*}+\left(n_{i}-1\right) b_{n}^{*} \text { com multiplicidade } 1
$$

e

$$
\lambda_{2_{n}}=a_{n}^{*}-b_{n}^{*} \text { com multiplicidade }\left(n_{i}-1\right)
$$

em que

$$
a_{n}^{*}=g_{1}\left(S_{n}, S_{0}\right)=\frac{\sigma_{e}^{2}}{S_{n}}-1+\frac{\sigma_{0}^{2}}{S_{n}}-\frac{S_{0}\left(\sigma_{e}^{2}+n_{i} S_{0}\right)}{S_{n}\left(S_{n}+n_{i} S_{0}\right)}
$$

e

$$
b_{n}^{*}=g_{2}\left(S_{n}, S_{0}\right)=\frac{\sigma_{0}^{2}}{S_{n}}-\frac{S_{0}\left(\sigma_{e}^{2}+n_{i} S_{0}\right)}{S_{n}\left(S_{n}+n_{i} S_{0}\right)} .
$$

Como $a_{n}^{*}$ e $b_{n}^{*}$ definidos acima constituem funções (q.c.) contínuas de $S_{n}$ e $S_{0}$, então

$$
\begin{aligned}
& a_{n}^{*}=g_{1}\left(S_{n}, S_{0}\right) \stackrel{\mathbb{P}}{\longrightarrow} g_{1}\left(\sigma_{e}^{2}, \sigma_{0}^{2}\right)=0, \\
& b_{n}^{*}=g_{2}\left(S_{n}, S_{0}\right) \stackrel{\mathbb{P}}{\longrightarrow} g_{2}\left(\sigma_{e}^{2}, \sigma_{0}^{2}\right)=0 .
\end{aligned}
$$

Nobre, Juvêncio S. 
Por fim, notemos que para $n_{i}$ fixado, $\lambda_{1_{n}}$ e $\lambda_{2_{n}}$ são funções contínuas (no caso funções lineares passando pela origem) de $a_{n}^{*}$ e $b_{n}^{*}$; então, por (B.41) e (B.42), concluímos que $\lambda_{i_{n}} \stackrel{\mathbb{P}}{\longrightarrow} 0(i=1,2)$, provando o resultado.

Por outro lado, se considerarmos, por exemplo que $\sup _{1 \leq s \leq n}\left\|\mathbf{x}_{i}\right\|$ é limitado, podemos provar que os autovalores de $\left(\mathbf{X}_{n}^{\top} \mathbf{V}_{n} \mathbf{X}_{n}\left(\mathbf{X}_{n}^{\top} \widehat{\mathbf{V}}_{n} \mathbf{X}_{n}\right)^{-1}-\mathbf{I}_{n}\right)$ também convergem em probabilidade para zero de forma similar ao caso anterior.

\section{B.9 Cálculo de $\mathbb{E}\left[J_{n \beta}^{*}\right]$}

Por simplicidade, consideremos que os efeitos aleatórios $b_{i 0}$ e $b_{i 1}$ são independentes. Sob $\mathcal{H}_{1}$ definida em (3.39), notemos que (3.42) pode ser reescrita como

$$
\begin{aligned}
T_{n \beta}^{*} & =\sum_{1 \leq r<s \leq n} \eta_{n r s} \psi\left(Y_{r}^{*}, Y_{s}^{*}, \boldsymbol{\beta}\right)=\sum_{1 \leq r<s \leq n} \eta_{n r s}\left(Y_{r}^{*}-\mathbf{x}_{r}^{* \top} \boldsymbol{\beta}\right)\left(Y_{s}^{*}-\mathbf{x}_{s}^{* \top} \boldsymbol{\beta}\right) \\
& =\sum_{1 \leq r<s \leq n} \eta_{n r s}\left(z_{r}^{*} b_{u(r) 1}+u_{r}\right)\left(z_{s}^{*} b_{u(s) 1}+u_{s}\right),
\end{aligned}
$$

com $z_{i}^{*}$ representando o valor da variável explicativa associada à inclinação aleatória do $i$-ésimo elemento (ordenados lexigrocaficamente) no modelo transformado

$$
\mathbf{Y}^{*}=\mathbf{X}^{*} \boldsymbol{\beta}+\mathbf{Z}^{*} \mathbf{b}+\mathbf{u}
$$

Desta forma, temos

$$
\begin{aligned}
\mathbb{E}\left[T_{n \beta}^{*}\right] & =\sum_{1 \leq r<s \leq n} \eta_{n r s} \mathbb{E}\left[\left(z_{r}^{*} b_{u(r) 1}+u_{r}\right)\left(z_{s}^{*} b_{u(s) 1}+u_{s}\right)\right] \\
& =\sum_{1 \leq r<s \leq n} \eta_{n r s} \operatorname{Cov}\left[z_{r}^{*} b_{u(r) 1}+u_{r}, z_{s}^{*} b_{u(s) 1}+u_{s}\right]
\end{aligned}
$$

Como $b_{i 0}$ e $b_{i 1}$ são independentes, então $\operatorname{Cov}\left[b_{u(i) 1}, u_{j}\right]=0$ para quaisquer $i, j$; portanto

$$
\begin{aligned}
\mathbb{E}\left[T_{n \beta}^{*}\right] & =\sum_{1 \leq r<s \leq n} \eta_{n r s} z_{r}^{*} z_{s}^{*} \operatorname{Cov}\left[b_{u(r) 1}, b_{u(s) 1}\right]=\sum_{1 \leq r<s \leq n} \eta_{n r s} z_{r}^{*} z_{s}^{*} \sigma_{1}^{2} \mathbb{1}\{u(s)=u(r)\} \\
& =\sigma_{1}^{2} \sum_{i=1}^{k}\left(\frac{n-n_{i}}{n_{i}-1}\right) \sum_{1 \leq j_{1}<j_{2} \leq n_{i}} z_{i j_{1}}^{*} z_{i j_{2}}^{*}=\sigma_{1}^{2} K_{n},
\end{aligned}
$$

que não necessariamente é positivo. Como $J_{n \beta}^{*}=T_{n \beta}^{*} / \sqrt{M_{n}}$, o resultado segue.

É interessante notar que quando $\mathbf{V}=\sigma_{e}^{2} \mathbf{I}_{n}$ com $z_{i j}=1$ para quaisquer $i, j$, ou seja, se o modelo em questão for um modelo do tipo (3.23), então $\mathbb{E}\left[T_{n \beta}^{*}\right]=\mathbb{E}\left[T_{n \beta}\right]=\frac{\sigma_{b}^{2}}{2}\left(n^{2}-\right.$ $\left.\sum_{i=1}^{k} n_{i}^{2}\right) \geq 0$, como discutido anteriormente. 


\section{B.10 Teorema 3.5}

Podemos reescrever (3.42) como

$$
\begin{aligned}
T_{n \beta}^{*} & =T_{n}^{*}(\boldsymbol{\beta})=\sum_{1 \leq r<s \leq n} \eta_{n r s}\left(Y_{r}^{*}-\mathbf{x}_{n r}^{* \top} \boldsymbol{\beta}\right)\left(Y_{s}^{*}-\mathbf{x}_{n s}^{* \top} \boldsymbol{\beta}\right) \\
& =\sum_{1 \leq r<s \leq n} \eta_{n r s}\left(Y_{r}^{*}-\mathbf{x}_{n r}^{* \top} \widehat{\boldsymbol{\beta}}_{n}^{a}\right)\left(Y_{s}^{*}-\mathbf{x}_{n s}^{* \top} \widehat{\boldsymbol{\beta}}_{n}^{a}\right)+\sum_{1 \leq r<s \leq n} \eta_{n r s} \mathbf{x}_{n s}^{* \top}\left(\widehat{\boldsymbol{\beta}}_{n}^{a}-\boldsymbol{\beta}\right)\left(Y_{r}^{*}-\mathbf{x}_{n r}^{* \top} \boldsymbol{\beta}\right) \\
& +\sum_{1 \leq r<s \leq n} \eta_{n r s}\left(Y_{s}^{*}-\mathbf{x}_{n s}^{* \top} \widehat{\boldsymbol{\beta}}_{n}^{a}\right) \mathbf{x}_{n r}^{* \top}\left(\widehat{\boldsymbol{\beta}}_{n}^{a}-\boldsymbol{\beta}\right) \\
& =T_{n \hat{\beta}^{a}}+T_{n 1}^{*}+T_{n 2}^{*},
\end{aligned}
$$

com

$$
\begin{aligned}
T_{n \hat{\beta}^{a}} & =\sum_{1 \leq r<s \leq n} \eta_{n r s}\left(Y_{r}^{*}-\mathbf{x}_{n r}^{* \top} \widehat{\boldsymbol{\beta}}_{n}^{a}\right)\left(Y_{s}^{*}-\mathbf{x}_{n s}^{* \top} \widehat{\boldsymbol{\beta}}_{n}^{a}\right) \\
T_{n 1}^{*} & =\sum_{1 \leq r<s \leq n} \eta_{n r s} \mathbf{x}_{n s}^{* \top}\left(\widehat{\boldsymbol{\beta}}_{n}^{a}-\boldsymbol{\beta}\right)\left(Y_{r}^{*}-\mathbf{x}_{n r}^{* \top} \boldsymbol{\beta}\right) \\
T_{n 2}^{*} & =\sum_{1 \leq r<s \leq n} \eta_{n r s}\left(Y_{s}^{*}-\mathbf{x}_{n s}^{* \top} \widehat{\boldsymbol{\beta}}_{n}^{a}\right) \mathbf{x}_{n r}^{* \top}\left(\widehat{\boldsymbol{\beta}}_{n}^{a}-\boldsymbol{\beta}\right) .
\end{aligned}
$$

O lema abaixo é uma extensão do Lema B.4.

Lema B.5. Consideremos o modelo (3.33) e $T_{n 1}^{*}$ e $T_{n 2}^{*}$ dados, respectivamente, em (B.46) e (B.47) e $\mathbf{x}_{n s}^{* \top}$ a s-ésima linha da matriz $\mathbf{X}_{n}^{*}$. Sob as suposições (3.34)-(3.36) e se existe uma constante $\gamma<\infty$ tal que

$$
\sup _{1 \leq s \leq n}\left\|\mathbf{x}_{n s}^{*}\right\| \leq \gamma
$$

então, para $i=1,2$, temos

$$
\frac{T_{n i}^{*}}{\sqrt{M_{n}}} \stackrel{\mathbb{P}}{\longrightarrow} 0
$$

Prova A demonstração é análoga a do Lema B.4.

Lembrando que $\widehat{\mathbf{V}}_{n}-\mathbf{V}_{n}=o_{p}(1)$ e que os autovalores da matriz $\mathbf{V}_{n} \widehat{\mathbf{V}}_{n}^{-1}-\mathbf{I}_{n}$ convergem em probabilidade para zero, é possível mostrar que $\widehat{\mathbf{V}}_{n}^{-1 / 2}-\mathbf{V}_{n}^{-1 / 2}=o_{p}(1)$, em que $\mathbf{V}_{n}^{-1 / 2}$ é obtida através da decomposição espectral. Denotando a $t$-ésima coluna de $\mathbf{I}_{n}$ por $\mathbf{I}_{n t}$, 
podemos reescrever $T_{n \hat{\beta}^{a}}$ como

$$
\begin{aligned}
T_{n \hat{\beta}^{a}} & =\sum_{1 \leq r<s \leq n} \eta_{n r s} \mathbf{I}_{n r}^{\top} \mathbf{V}_{n}^{-1 / 2}\left(\mathbf{Y}_{n}-\mathbf{X}_{n} \widehat{\boldsymbol{\beta}}_{n}^{a}\right) \mathbf{I}_{n s}^{\top} \mathbf{V}_{n}^{-1 / 2}\left(\mathbf{Y}_{n}-\mathbf{X}_{n} \widehat{\boldsymbol{\beta}}_{n}^{a}\right) \\
& =\sum_{1 \leq r<s \leq n} \eta_{n r s} \mathbf{I}_{n r}^{\top} \widehat{\mathbf{V}}_{n}^{-1 / 2}\left(\mathbf{Y}_{n}-\mathbf{X}_{n} \widehat{\boldsymbol{\beta}}_{n}^{a}\right) \mathbf{I}_{n s}^{\top} \widehat{\mathbf{V}}_{n}^{-1 / 2}\left(\mathbf{Y}_{n}-\mathbf{X}_{n} \widehat{\boldsymbol{\beta}}_{n}^{a}\right) \\
& +o_{p}(1) \sum_{1 \leq r<s \leq n} \eta_{n r s} \mathbf{I}_{n r}^{\top}\left(\mathbf{Y}_{n}-\mathbf{X}_{n} \widehat{\boldsymbol{\beta}}_{n}^{a}\right) \mathbf{I}_{n s}^{\top}\left(\mathbf{Y}_{n}-\mathbf{X}_{n} \widehat{\boldsymbol{\beta}}_{n}^{a}\right) \\
& =T_{n \hat{\beta}^{a}}^{*}+o_{p}(1) \sum_{1 \leq r<s \leq n} \eta_{n r s} \hat{e}_{n r} \hat{e}_{n s},
\end{aligned}
$$

em que $\hat{e}_{n i}$ denota o $i$-ésimo elemento do vetor de resíduos marginais $\widehat{\mathbf{e}}_{n}$. Além disso, é possível mostrar que $\sum_{1 \leq r<s \leq n} \eta_{n r s} \hat{e}_{n r} \hat{e}_{n s}=O_{p}\left(\sqrt{M_{n}}\right)$, de forma que considerando (3.43), as decomposições (B.44) e (B.49), o Lema B.5 e o Teorema de Slutsky, concluímos que sob $\mathcal{H}_{0}: \sigma_{0}^{2}>0$ e $\sigma_{1}^{2}=0$

$$
J_{n \hat{\beta}^{a}}^{*}=\frac{T_{n \hat{\beta}^{a}}^{*}}{\sqrt{M_{n}}} \stackrel{\mathcal{D}}{\longrightarrow} \mathcal{N}(0,1),
$$

quando $k \rightarrow \infty$.

\section{B.11 Teorema 3.6}

Inicialmente, consideremos a matriz $\mathbf{V}_{n}$ conhecida; levando (B.43) em consideração sob a seqüência $\mathcal{H}_{1 n}: \Omega \mathrm{NND}$, com $\sigma_{0}^{2}>0$ e $\sigma_{1}^{2}=\delta^{2} / \sqrt{n}$, temos

$$
\begin{aligned}
T_{n \hat{\beta}^{a}} & =\sum_{1 \leq r<s \leq n} \eta_{n r s}\left(\left\{\mathbf{x}_{n r}^{* \top} \boldsymbol{\beta}+u_{r}\right\}+z_{r}^{*} b_{u(r) 1}-\mathbf{x}_{n r}^{* \top} \widehat{\boldsymbol{\beta}}_{n}^{a}\right)\left(\left\{\mathbf{x}_{n s}^{* \top} \boldsymbol{\beta}+u_{s}\right\}+z_{s}^{*} b_{u(s) 1}-\mathbf{x}_{n s}^{* \top} \widehat{\boldsymbol{\beta}}_{n}^{a}\right) \\
& =T_{n \hat{\beta}^{a}}^{0}+D_{n 1}+D_{n 2}+D_{n 3},
\end{aligned}
$$

em que

$$
\begin{aligned}
T_{n \hat{\beta}^{a}}^{0} & =\sum_{1 \leq r<s \leq n} \eta_{n r s}\left(\mathbf{x}_{n r}^{* \top} \boldsymbol{\beta}+u_{r}-\mathbf{x}_{n r}^{* \top} \widehat{\boldsymbol{\beta}}_{n}^{a}\right)\left(\mathbf{x}_{n s}^{* \top} \boldsymbol{\beta}+u_{s}-\mathbf{x}_{n s}^{* \top} \widehat{\boldsymbol{\beta}}_{n}^{a}\right), \\
D_{n 1} & =\sum_{1 \leq r<s \leq n} \eta_{n r s} z_{r}^{*} b_{u(r) 1}\left(\mathbf{x}_{n s}^{* \top}\left(\boldsymbol{\beta}-\widehat{\boldsymbol{\beta}}_{n}^{a}\right)+u_{s}\right), \\
D_{n 2} & =\sum_{1 \leq r<s \leq n} \eta_{n r s} z_{s}^{*} b_{u(s) 1}\left(\mathbf{x}_{n r}^{* \top}\left(\boldsymbol{\beta}-\widehat{\boldsymbol{\beta}}_{n}^{a}\right)+u_{r}\right), \\
D_{n 3} & =\sum_{1 \leq r<s \leq n} \eta_{n r s} z_{r}^{*} z_{s}^{*} b_{u(r) 1} b_{u(s) 1} .
\end{aligned}
$$

Nobre, Juvêncio S. 
Sob $\mathcal{H}_{0}, T_{n \hat{\beta}^{a}}=T_{n \hat{\beta}^{a}}^{0}$ quase certamente e portanto, pelo Teorema 3.5, obtemos

$$
\frac{T_{n \hat{\beta}^{a}}^{0}}{\sqrt{M_{n}}} \stackrel{\mathcal{D}}{\longrightarrow} \mathcal{N}(0,1)
$$

quando $k \rightarrow \infty$.

Podemos reescrever $D_{n 1}$ como

$$
\begin{aligned}
D_{n 1} & =\sum_{1 \leq r<s \leq n} \eta_{n r s} z_{r}^{*} b_{u(r) 1} \mathbf{x}_{n s}^{* \top}\left(\boldsymbol{\beta}-\widehat{\boldsymbol{\beta}}_{n}^{a}\right)+\sum_{1 \leq r<s \leq n} \eta_{n r s} z_{r}^{*} b_{u(r) 1} u_{s} \\
& =\left(\boldsymbol{\beta}-\widehat{\boldsymbol{\beta}}_{n}^{a}\right)^{\top} \sum_{1 \leq r<s \leq n} \eta_{n r s} z_{r}^{*} b_{u(r) 1} \mathbf{x}_{n s}+\sum_{1 \leq r<s \leq n} \eta_{n r s} z_{r}^{*} b_{u(r) 1} u_{s} .
\end{aligned}
$$

Analogamente, é possível mostrar que

$$
D_{n 2}=\left(\boldsymbol{\beta}-\widehat{\boldsymbol{\beta}}_{n}^{a}\right)^{\top} \sum_{1 \leq r<s \leq n} \eta_{n r s} z_{s}^{*} b_{u(s) 1} \mathbf{x}_{n r}+\sum_{1 \leq r<s \leq n} \eta_{n r s} z_{s}^{*} b_{u(s) 1} u_{r} .
$$

O lema abaixo é uma extensão dos Lemas B.4 e B.5.

Lema B.6. Consideremos o modelo (3.41) sob a seqüência $\mathcal{H}_{1 n}: \Omega \quad N N D$, com $\sigma_{0}^{2}>$ 0 e $\sigma_{1}^{2}=\delta^{2} / \sqrt{n}$, as estatisticas $D_{n 1}$ e $D_{n 2}$ dadas, respectivamente, em (B.53) e (B.54), $\mathbf{x}_{s}^{* \top}$ a s-ésima linha da matriz $\mathbf{X}^{*}$ e $z_{s}^{*}$ definida em (3.48). Sob as suposições (3.34)-(3.36) e se existem constantes $\gamma_{1}<\infty$ e $\gamma_{2}<\infty$ tais que

$$
\sup _{1 \leq s \leq n}\left\|\mathbf{x}_{n s}^{*}\right\| \leq \gamma_{1} \quad e \quad \sup _{1 \leq s \leq n}\left\|z_{n s}^{*}\right\| \leq \gamma_{2}
$$

Para $i=1,2$, temos

$$
\frac{D_{n i}}{\sqrt{M_{n}}} \stackrel{\mathbb{P}}{\longrightarrow} 0
$$

\section{Prova}

A demonstração é análoga às demonstrações dos Lemas B.4 e B.5, dado que estamos supondo independência entre $b_{i 0}$ e $b_{i 1}$.

Por outro lado, podemos decompor o terceiro termo da decomposição (B.51) como

$$
\begin{aligned}
D_{n 3} & =\sum_{1 \leq r<s \leq n} \eta_{n r s} z_{r}^{*} z_{s}^{*} b_{u(r) 1} b_{u(s) 1} \\
& =\sum_{i=1}^{k}\left(\frac{n-n_{i}}{n_{i}-1}\right) b_{u(i) 1}^{2} \sum_{1 \leq j_{1}<j_{2} \leq n} z_{j_{1}}^{*} z_{j_{2}}^{*}-\sum_{1 \leq i<i^{\prime} \leq n} b_{u(i) 1} b_{u\left(i^{\prime}\right) 1} \sum_{j_{1}=1}^{n_{i}} \sum_{j_{2}=1}^{n_{i^{\prime}}} z_{j_{1}}^{*} z_{j_{2}}^{*} \\
& =D_{n 31}-D_{n 32},
\end{aligned}
$$

Nobre, Juvêncio S. 
$\operatorname{com} D_{n 31}=\sum_{i=1}^{k}\left(\frac{n-n_{i}}{n_{i}-1}\right) b_{u(i) 1}^{2} \sum_{1 \leq j_{1}<j_{2} \leq n} z_{j_{1}}^{*} z_{j_{2}}^{*}$ e $D_{n 32}=\sum_{1 \leq i<i^{\prime} \leq n} b_{u(i) 1} b_{u\left(i^{\prime}\right) 1} \sum_{j_{1}=1}^{n_{i}} \sum_{j_{2}=1}^{n_{i^{\prime}}} z_{j_{1}}^{*} z_{j_{2}}^{*}$.

Então $\mathbb{E}\left[D_{n 32}\right]=0$ e

$$
\begin{aligned}
\mathbb{E}\left[D_{n 3}\right] & =\mathbb{E}\left[D_{n 31}\right]=\sigma_{1}^{2} \sum_{i=1}^{k}\left(\frac{n-n_{i}}{n_{i}-1}\right) \sum_{1 \leq j_{1}<j_{2} \leq n_{i}} z_{i j_{1}}^{*} z_{i j_{2}}^{*} \\
& =\frac{\delta^{2}}{\sqrt{n}} K_{n}=O\left(n^{3 / 2}\right),
\end{aligned}
$$

considerando (B.57). Conseqüentemente

$$
\lim _{n \rightarrow \infty} \frac{\mathbb{E}\left[D_{n 3}\right]}{\sqrt{M_{n}}}=\lim _{n \rightarrow \infty} \frac{\mathbb{E}\left[D_{n 31}\right]}{\sqrt{M_{n}}}=\delta^{2} \lim _{n \rightarrow \infty} \frac{K_{n}}{\sqrt{n M_{n}}}=\kappa \frac{\delta^{2}}{\sqrt{\lambda^{*}}} .
$$

Lembrando que $b_{1}, \ldots, b_{k}$ são iid e que os $n_{i}$ 's são limitados e considerando que $\mathbb{E}\left[b_{i 1}^{4}\right]<\infty$ (implicando pela contigüidade da seqüência $\mathcal{H}_{1 n}$ que $\mathbb{E}\left[b_{i 1}^{4}\right]=o(1)$ ), então podemos mostrar de forma análoga àquela empregada na Seção B.7 que

$$
\frac{D_{n 31}}{\sqrt{M_{n}}} \stackrel{\mathbb{P}}{\longrightarrow} \lim _{n \rightarrow \infty} \frac{\mathbb{E}\left[D_{n 31}\right]}{\sqrt{M_{n}}}=\kappa \frac{\delta^{2}}{\sqrt{\lambda^{*}}}
$$

e

$$
\frac{D_{n 32}}{\sqrt{M_{n}}} \stackrel{\mathbb{P}}{\longrightarrow} 0
$$

Logo,

$$
\frac{D_{n 3}}{\sqrt{M_{n}}}=\frac{D_{n 31}}{\sqrt{M_{n}}}+\frac{D_{n 32}}{\sqrt{M_{n}}} \stackrel{\mathbb{P}}{\longrightarrow} \kappa \frac{\delta^{2}}{\sqrt{\lambda^{*}}} .
$$

Pela decomposição (B.51), por (B.56) e (B.58), pelo Lema (B.6) e pelo Teorema de Slutsky, concluímos que

$$
\frac{T_{n \hat{\beta}^{a}}}{\sqrt{M_{n}}} \stackrel{\mathcal{D}}{\longrightarrow} \mathcal{N}\left(\kappa \frac{\delta^{2}}{\sqrt{\lambda^{*}}}, 1\right) .
$$

De forma similar à empregada na seção anterior, especificamente tendo em vista a identidade (B.49), é possível mostrar que

$$
J_{n \hat{\beta}^{a}}^{*}-\frac{T_{n \hat{\beta}^{a}}}{\sqrt{M_{n}}} \stackrel{\mathbb{P}}{\longrightarrow} 0,
$$

e usando o Teorema de Slutsky, podemos concluir finalmente que

$$
J_{n \hat{\beta}^{a}}^{*} \stackrel{\mathcal{D}}{\longrightarrow} \mathcal{N}\left(\kappa \frac{\delta^{2}}{\sqrt{\lambda^{*}}}, 1\right) .
$$

Nobre, Juvêncio S. 


\section{Referências Bibliográficas}

[1] Afiune, J.Y. (2000). Avaliação ecocardiográfica evolutiva de recém-nascidos pré-termo, do nascimento até o termo. Tese de doutorado. Instituto da Criança da Faculdade de Medicina, Universidade de São Paulo: São Paulo.

[2] Afiune, J.Y, Singer, J.M. and Leone, C.R. (2005). Evolução ecocardiográfica de recémnascidos com persistência do canal arterial. Jornal de Pediatria, 81, 454-460.

[3] Akritas, M. and Arnold, S. (2000). Asymptotics for analysis of variance when the number of levels is large. Journal of the American Statistical Association, 95, 212-226.

[4] Andrews, D.W.K. (2001). Testing when a parameter is on the boundary of the maintained hypothesis. Econometrica, 69, 683-734.

[5] Arellano-Valle, R.B., Bolfarine, H. and Lachos, V.H. (2005). Skew-Normal linear mixed models. Journal of Data Science, 3, 415-438.

[6] Azzalini, A. and Capitanio, A. (2003). Distributions generated by perturbation of symmetry with emphasis on a multivariate skew $t$-distribution. Journal of the Royal Statistical Society B, 65, 367-389.

[7] Bell, C.B., Blackwell, D. and Breiman, L. (1960). On completeness of order statistics. Annals of Mathematics and Statistics, 31, 794-797.

[8] Berk, R.H. (1966). Limiting behaviour of posterior distributions when the model is incorrect. The Annals of Mathematical Statistics, 37, 51-58.

[9] Bertail, P. and Tressou, J. (2006). Incomplete generalized $U$-statistics for food risk assessment. Biometrics, 62, 66-74.

[10] Brown, K.G. (1976). Asymptotic behaviour of MINQUE-type estimators of variance components. Annals of Statistics, 4, 746-754. 
[11] Butler, S.M. and Louis, T.A. (1992). Random effects models with non-parametric priors. Statistics in Medicine, 11, 1981-2000.

[12] Chow, Y.S. and Teicher, H. (2003). Probability Theory: Independence, Interchangeability, Martingales, 3rd edition. New York: Springer.

[13] Cox, D.R. and Hinkley, E.J. (1974). Theoretical Statistics. London: Chapman \& Hall.

[14] Crainiceanu, C.M. and Ruppert, D. (2004). Likelihood ratio tests in linear mixed models with one variance component. Journal of the Royal Statistical Society B, 66, 165-185.

[15] Davison, A.C. and Hinkley, D.V. (1997). Bootstrap Methods and Their Applications. Cambridge: Cambridge University Press.

[16] Demidenko, E. (2004). Mixed Models: Theory and Applications. New York: John Wiley \& Sons.

[17] Demidenko, E. and Stukel, T. (2002). Efficient estimation of general mixed effects models. Journal of Statistical Planning and Inference, 104, 197-219.

[18] Diggle, P.J., Heagerty, P., Liang, K.Y. and Zeger, S.L. (2002). Analysis of Longitudinal Data, 2nd edition. Oxford: Oxford University Press.

[19] Donner, A. and Koval, J.J. (1989). The effect of imbalance on significance-testing in one-way model II analysis of variance. Communications in Statistics - Theory and Methods, 18, 1239-1250.

[20] Dvoretzky, A. (1972). Asymptotic normality for sums of dependent random variables. In Proceedings of the sixth Berkeley symposium on Mathematical Statistics and Probability, vol 2, 513-536. Berkeley: University of California Press.

[21] Fellner, W.H. (1986). Robust estimation of variance components. Technometrics, 28, 51-60.

[22] Feng, Z. and McCulloch, C.E. (1992). Statistical inference using maximum likelihood estimation and the generalized likelihood ratio when the true parameter is on the boundary of the parameter space. Statistic \& Probability Letters, 13, 325-332.

[23] Fraser, D.A.S. (1954). Completeness of order statistics. Canadian Journal of Mathematics, 6, 42-45. 
[24] Giampaoli, V. (1999). Inferência estatística para modelos lineares com restrição nos parâmetros em condições regulares e não regulares. Tese de doutorado. IME/USP: São Paulo.

[25] Giampaoli, V. and Singer, J.M. (2007). Generalized likelihood ratio tests for variance components in linear mixed models (submetido para publicação).

[26] Godoi, L. G. (2007). A distribuição t-assimétrica univariada: propriedades e inferência. Dissertação de mestrado. IME/USP: São Paulo.

[27] Hall, D. and Praestgaard, J.T. (2001). Order-restricted score tests for homogeneity in generalised linear and nonlinear mixed models. Biometrika, 88, 739-751.

[28] Hall, P. and Heyde, C.C. (1980). Martingale Limit Theory and its Applications. New York: Academic Press.

[29] Halmos, P.R. (1946). The theory of unbiased estimation. Annals of Mathematical Statistics, 17, 34-43.

[30] Harville, D. (1997). Matrix Algebra from a Statistician's Perpesctive. New York: Springer.

[31] Hilden-Minton, J.A. (1995). Multilevel diagnostics for mixed and hierarchical linear models. PhD Thesis. University of California: Los Angeles.

[32] Hoeffding, W. (1948). A class of statistics with asymptotically normal distribution. Annals of Mathematical Statistics, 19, 293-325.

[33] Hoeffding, W. (1961). The strong law of large number for U statistics. Institute of Statistics Mimeo Series \# 302, University of North Carolina.

[34] Huggins, R.M. (1993). A robust approach to the analysis of repeated measures. Biometrics, 49, 715-720.

[35] Jiang, J. (1997). Wald consistency and the method of sieves in REML estimation. The Annals of Statistics, 4, 1781-1803.

[36] Jiang, J. (1998). Consistent estimators in generalized linear mixed models. Journal of the American Statistical Association, 93, 720-729. 
[37] Jiang, J. (2007). Linear and Generalized Linear Mixed Models and Their Applications. New York: Springer-Verlag.

[38] Jiang, J. and Zhang, W. (2001). Robust estimation in generalised linear mixed models. Biometrika, 88, 753-765.

[39] Jurečková, J. and Sen, P.K. (1996). Robust Statistical Procedures: Asymptotics and Interrelations. New York: John Wiley \& Sons.

[40] Khuri, A.I., Mathew, T. and Sinha, B.K. (1998). Statistical Tests for Mixed Linear Models. New York: John Wiley \& Sons.

[41] Kowalski, J. (2001). A nonparametric approach to translating gene region heterogeneity associated with phenotype into location heterogeneity. Bionformatics, 17, 775-790.

[42] Kowalski, J., Pagano, M. and DeGruttola, V. (2002). A nonparametric test of gene region heterogeneity associated with phenotype. Journal of the American Statistical Association, 97, 398-408.

[43] Laird, N.M. and Ware, J.M. (1982). Random-effects models for longitudinal data. Biometrics 38, 963-974.

[44] Lee, A.J. (1990). U-statistics: Theory and Practice. New York: Marcel Dekker.

[45] Lee, J. (2003). The effect of design imbalance on the power of the $F$-test of a variance component in the one-way random model. Biometrical Journal, 45, 238-248.

[46] Lee, Y., Nelder, J.A. and Pawitan, Y. (2006). Generalized Linear Models with Random Effects: Unified Analysis via H-likelihood. London: Chapman \& Hall.

[47] Lehmann, E.L. (1998). Elements of Large-Sample Theory. New York: Springer-Verlag.

[48] Lehmann, E.L. and Casella, G. (1998). Theory of Point Estimation, 2nd edition. New York: Springer-Verlag.

[49] Lehmann, E.L. and Romano, J.P. (2005). Testing Statistical Hypothesis, 3rd edition. New York: Springer-Verlag.

[50] Lin, X. (1997). Variance component testing in generalised linear models with random effects. Biometrika, 84, 309-326. 
[51] Lin, X. and Carroll, R.J. (1999). SIMEX variance component tests in generalized linear mixed measurement error models. Biometrics, 55, 613-619.

[52] Lindsey, J.K. (1993). Models for Repeated Measurements. Oxford: Oxford University Press.

[53] Longford, N.T. (1994). Random Coefficient Models. Oxford: Oxford University Press.

[54] Ma, Y., Genton, M.G. and Davidian, M. (2004). Linear mixed effects models with flexible generalized skew-elliptical random effects. In Skew-Elliptical Distributions and Their Applications. Ed. Marc G. Genton. New York: Chapman \& Hall.

[55] McCulloch, C.E. and Searle, S.R. (2001). Generalized, Linear, and Mixed Models. New York: John Wiley \& Sons.

[56] Mehrotra, D.V. (1997). Non-iterative robust estimators of variance components in within-subjects design. Statistics in Medicine, 16, 1465-1479.

[57] Molenberghs, G. and Verbeke, G. (2005). Models for Discrete Longitudinal Data. New York: Springer-Verlag.

[58] Morrell, C.H. (1998). Likelihood ratio testing of variance components in the linear mixed-effects model using restricted maximum likelihood. Biometrics, 54, 1560-1568.

[59] Müller, C.H. and Uhlig, S. (2001). Estimation of variance components with high breakdown point and high efficiency. Biometrika, 88, 353-366.

[60] Nobre, J.S. (2004). Métodos de diagnóstico para modelos lineares mistos. Dissertação de mestrado. IME/USP: São Paulo.

[61] Nobre, J.S. and Singer, J.M. (2007). Residual analysis for linear mixed models. Biometrical Journal. (aceito)

[62] Pendergast, J.F. and Broffitt, J.D. (1985). Robust estimation in growth curve models. Communication in Statistics-Theory and Methods, 14, 1919-1939.

[63] Pinheiro, J.C. and Bates, D.M. (2000). Mixed-Effects in S and S-PLUS. New York: Springer-Verlag.

[64] Pinheiro, H.P., Pinheiro, A. and Sen, P.K. (2005). Comparison of genomic sequences using Hamming distance. Journal of Statistical Planning and Inference, 130, 325-339. 
[65] Pinheiro, A., Sen, P.K. and Pinheiro, H.P. (2007). Decomposability of high-dimensional diversity measures: Quasi $U$-statistics, martingales and nonstandard asymptotics (submetido para publicação).

[66] Press, S.J. (2005). Applied Multivariate Analysis: Using Bayesian and Frequentist Methods of Inference, 2nd edition. New York: Dover.

[67] R Development Core Team (2006). R: A language and environment for statistical computing. R Foundation for Statistical Computing, Vienna, Austria. ISBN 3-900051-07-0, URL http://www.R-project.org.

[68] Rao, C.R. (1970). Estimation of heteroscedastic variances in linear models. Journal of the American Statistical Association, 65, 161-172.

[69] Rao, C.R. (1971a). Estimation of variance and covariance components - MINQUE theory. Journal of Multivariate Analysis, 1, 257-275.

[70] Rao, C.R. (1971b). Minimum variance quadratic unbiased estimation of variance components. Journal of Multivariate Analysis, 1, 445-456.

[71] Rao, C.R. (1972). Estimation of variance and covariance components in linear models. Journal of the American Statistical Association, 67, 112-115.

[72] Rao, C.R. and Kleffe, J. (1988). Estimation of Variance Components and Applications. Amsterdam: North-Holland.

[73] Richardson, A.M. and Welsh, A.H. (1995). Robust restricted maximum likelihood in mixed linear models. Biometrics, 51, 1429-1439.

[74] Rocha, F.M.M. (2004). Seleção de estruturas de covariância em dados com medidas repetidas. Dissertação de Mestrado. IME/USP, São Paulo.

[75] Rocha, F.M.M. and Singer, J.M. (2007). Selection of fixed and random effects in linear mixed models (sob revisão).

[76] Savalli, C., Paula, G.A. and Cysneiros, F.J.A. (2006). Assesment of variance components in elliptical linear mixed models. Statistical Modelling, 6, 59-76.

[77] Schaid, D.J., McDonnell, S.K., Hebbring, S.J., Cunningham, J.M. and Thibodeau, S. N. (2005). Nonparametric tests of association of multiple genes with human disease. American Journal Human Genetics, 76, 780-793. 
[78] Schervish, M.J. (1995). Theory of Statistics. New York: Springer-Verlag.

[79] Self, S.G. and Liang, K.Y. (1987). Asymptotic properties of maximum likelihood estimators and likelihood ratio tests under nonstandard conditions. Journal of the American Statistical Association, 82, 605-610.

[80] Sen, P.K. (1981). Sequential Nonparametrics: Invariance Principles and Statistical Inference. New York: John Wiley \& Sons.

[81] Sen, P.K. (1999). Utility-oriented Simpson-type indexes and inequalities measures. Bulletin of the Calcutta Statistical Association, 49, 1-22.

[82] Sen, P.K. (2006). Robust statistical inference for high-dimensional data models with application to genomics. Austrian Journal of Statistics, 35, 197-214.

[83] Sen, P.K. and Singer, J.M. (1993). Large Sample Methods in Statistics: An Introduction with Applications. New York: Chapman \& Hall.

[84] Serfling, R.J. (1980). Approximation Theorems of Mathematical Statistics. New York: John Wiley \& Sons.

[85] Shao, J. and Tu, D. (1995). The Jackknife and Bootstrap. New York: Springer-Verlag.

[86] Shiryayvev, A.N. (1983). Probability. New York: Springer-Verlag.

[87] Silvapulle, M.J. and Silvapulle, P. (1995). A score test against one-sided alternatives. Journal of the American Statistical Association, 90, 342-349.

[88] Silvapulle, M.J. and Sen, P. K. (2005). Constrained Statistical Inference. New York: John Wiley \& Sons.

[89] Singer, J.M. and Andrade, D.F. (2000). Analysis of longitudinal data. In Handbook of Statistics, Volume 18: Bio-environmental and Public Health Statistics. Eds. P.K. Sen and C.R. Rao. Amsterdam: North Holland, 115-160.

[90] Singer, J.M., Nobre, J.S. and Sef, H.C. (2004). Regression models for pretest/posttest data in blocks. Statistical Modelling, 4, 324-338.

[91] Sinha, S. (2004). Robust analysis in generalized linear mixed models. Journal of the American Statistical Association, 99, 451-460. 
[92] Stram, D.O. and Lee, J.W. (1994). Variance components testing in the longitudinal mixed effects model. Biometrics, 50, 1171-1177.

[93] Stram, D.O. and Lee, J.W. (1995). Correction to variance components testing in the longitudinal mixed effects model. Biometrics, 51, 1196.

[94] Tzeng, J.Y., Byerley, W., Devlin, B., Roeder, K. and Wasserman, L. (2003). Outlier detection and false discovery for whole-genome DNA matching. Journal of the American Statistical Association, 98, 236-246.

[95] Van der Vaart, A.W. (1998). Asymptotic Statistics. Cambridge: Cambridge University Press.

[96] Van Zwet, W.R. (1984). A Berry-Esseen bound for symmetric statistics. Z. Wahrsch. und Verw. Gebiete, 66, 425-440.

[97] Verbeke, G. and Lesaffre, E. (1996). Large samples properties of the maximum likelihood estimators in linear mixed models with misspecified random-effects distributions. Technical report, Biostatistical Centre for Clinical Trials, Catholic University of Leuven, Belgium.

[98] Verbeke, G. and Lesaffre, E. (1997). The effect of misspecifying the random-effects distributions in linear mixed models for longitudinal data. Computational Statistics \& Data Analysis, 23, 541-556.

[99] Verbeke, G. and Molenberghs, G. (2000). Linear Mixed Models for Longitudinal Data. New York: Springer-Verlag.

[100] Verbeke, G. and Molenberghs, G. (2003). The use of score tests for inference on variance components. Biometrics, 59, 254-262.

[101] Vonesh, E.F., Wang, H., Nie, L. and Majumdar, D. (2002). Conditional second-order generalized estimating equations for generalized linear and nonlinear mixed-effects models. Journal of the American Statistical Association, 97, 271-283.

[102] Vu, H.T. and Zhou, S. (1997). Generalization of likelihood ratio tests under nonstardard conditions. The Annals of Statistics, 25, 897-916.

[103] Welsh, A.H. and Richardson, A.M. (1997). Approaches to the robust estimation of mixed models. In Handbook of Statistics, Volume 15: Robust Inference. Eds. G.S. Maddala and C.R. Rao. Amsterdam: North-Holland, 343-384. 
[104] Yang, Y.X., Xu, T.H. and Song, L.J. (2005). Robust estimation of variance components with application in global positioning system network adjustment. Journal of Surveying Engineering, 131, 107-112.

[105] Yau, K.K.W. and Kuk, A.Y.C. (2002). Robust estimation in generalized linear mixed models. Journal of the Royal Statistical Society B, 64, 101-117.

[106] Zhu, Z. and Fung, W.K. (2004). Variance component testing in semiparametric mixed models. Journal of Multivariate Analysis, 91, 107-118. 